\title{
Next Generation Hydrogen Station Composite Data Products: Retail Stations Data through Quarter 4 of 2017
}

\author{
Sam Sprik, Jennifer Kurtz, Genevieve Saur, \\ Shaun Onorato, Matt Ruple, and \\ Chris Ainscough \\ May 2018
}




\section{Hydrogen Station Project Partners}
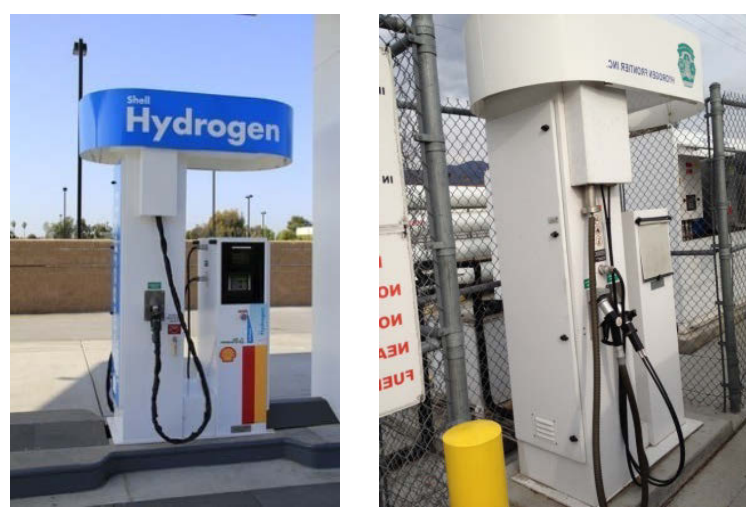

Los Angeles

- FirstElement Fuel

- Gas Technology Institute

- Linde

- H2 Frontier

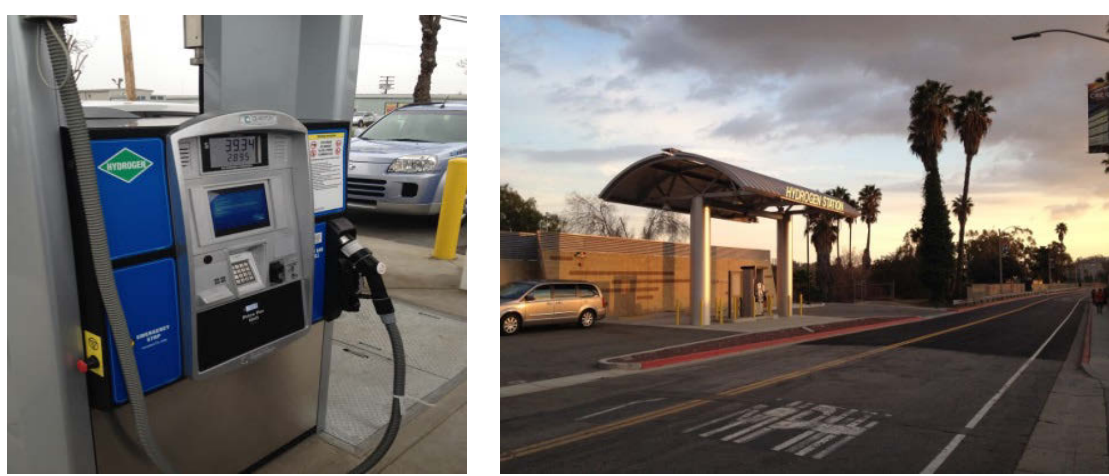

- Proton OnSite

- Shell

- IPHE and HySUT

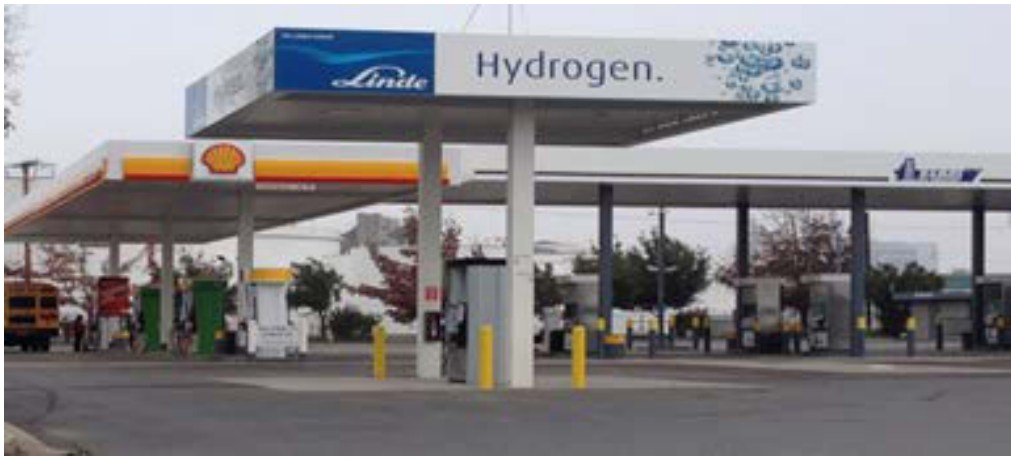




\section{Analysis Categories}

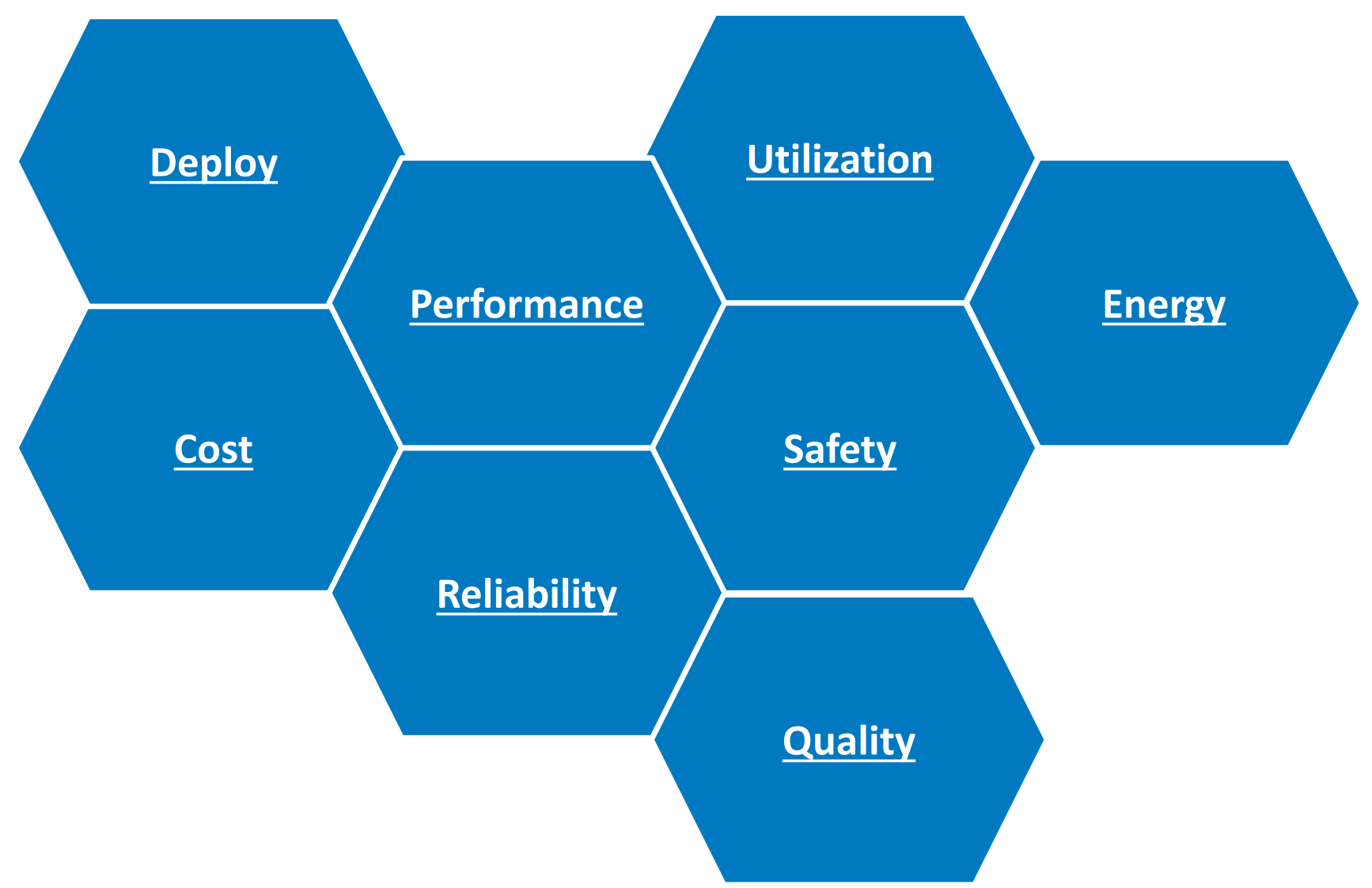




\section{CDP-INFR-10}

\section{Cumulative Number of Stations}

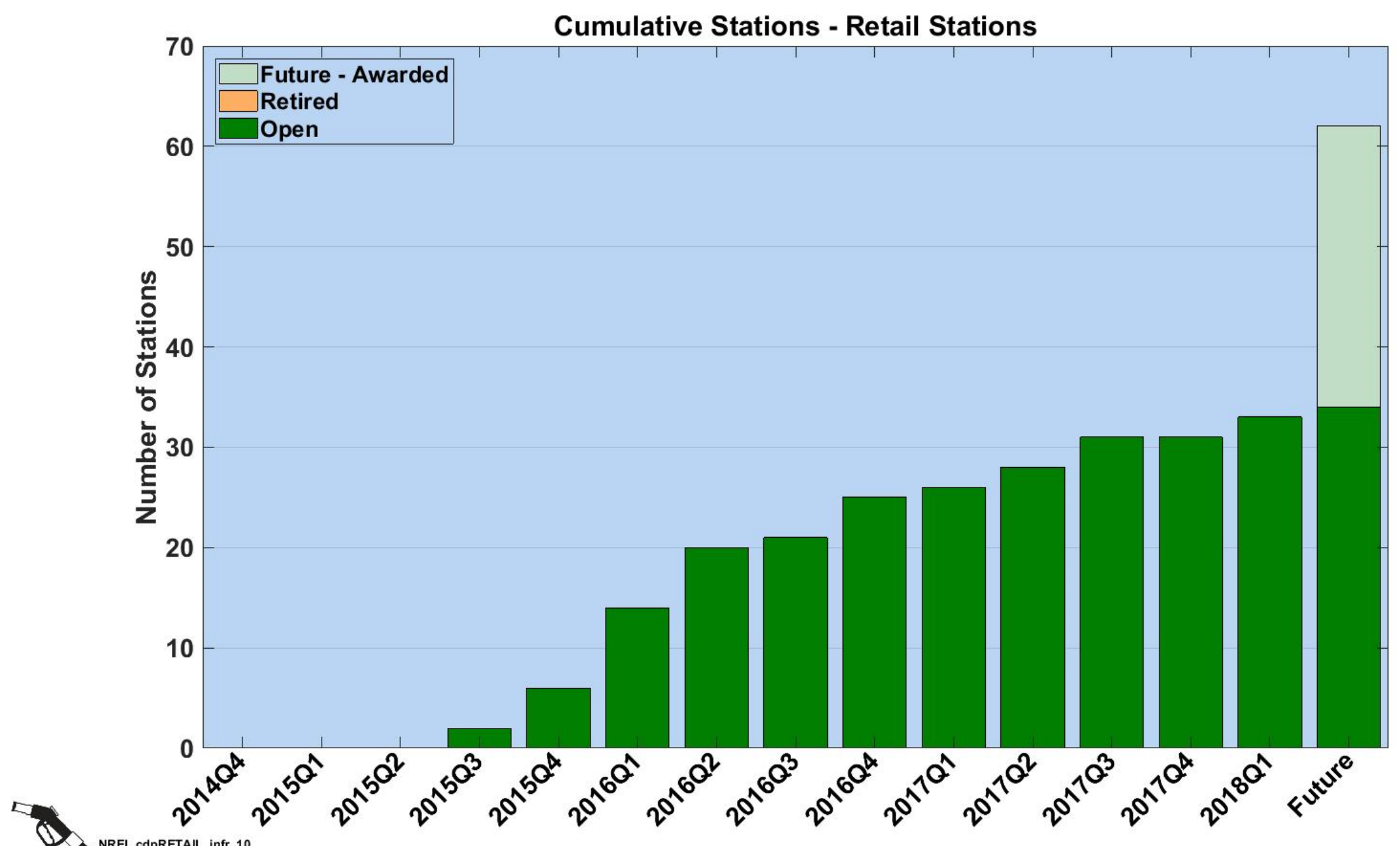




\section{CDP-INFR-11 \\ Hydrogen Stations by Type}

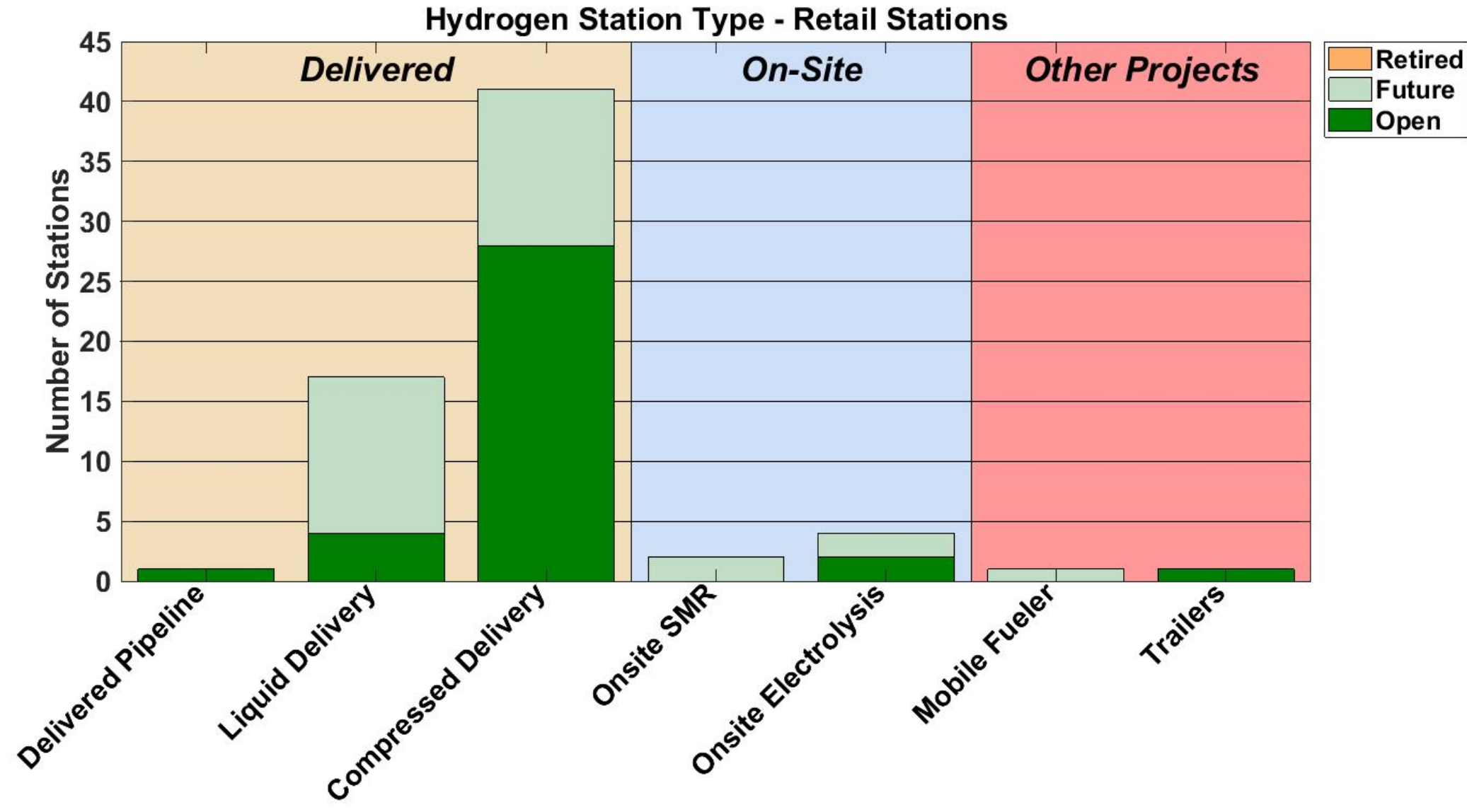




\section{CDP-INFR-27 \\ Hydrogen Station Timeline}

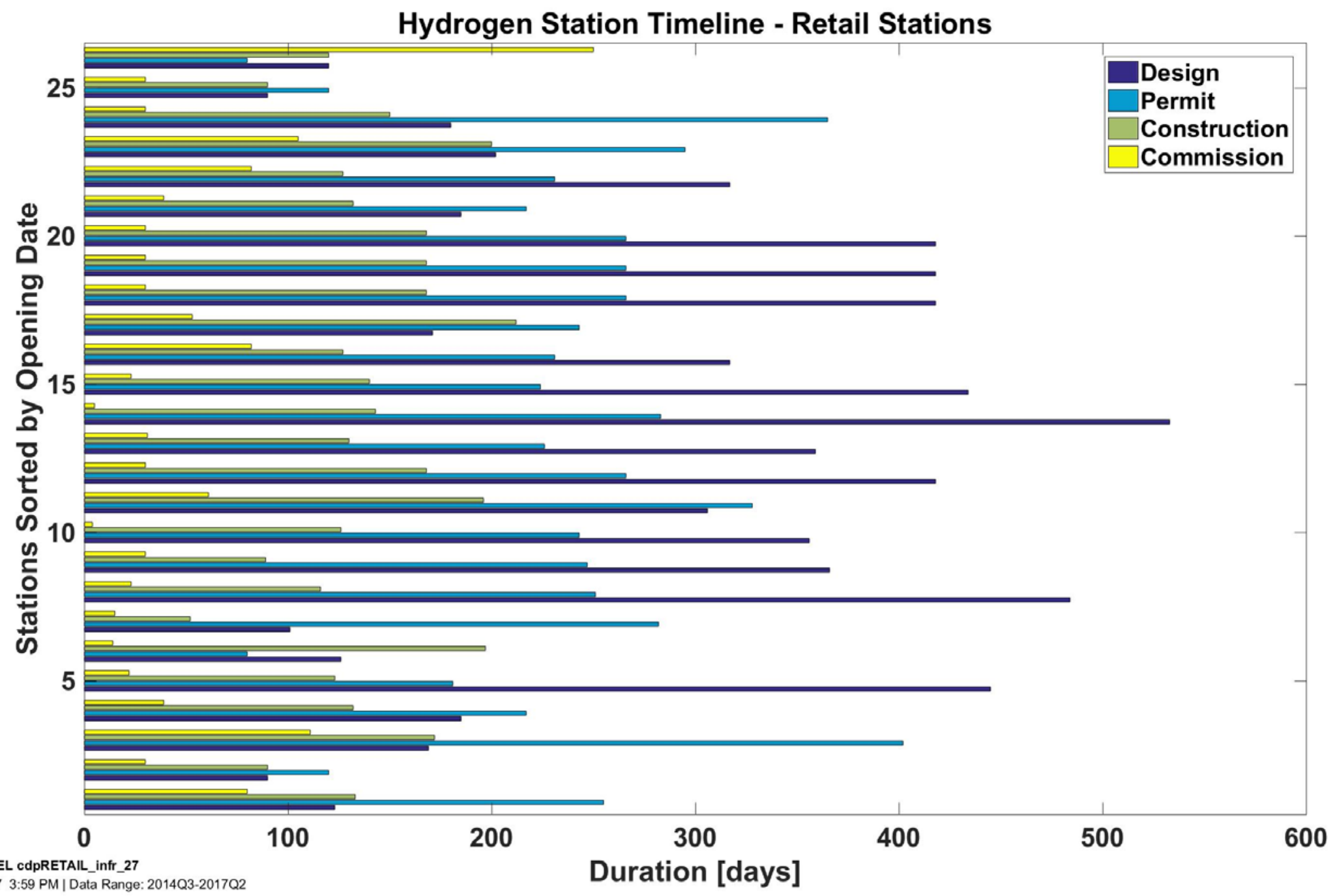


Safety 


\section{Safety Reports Primary Factors}

CDP-INFR-31

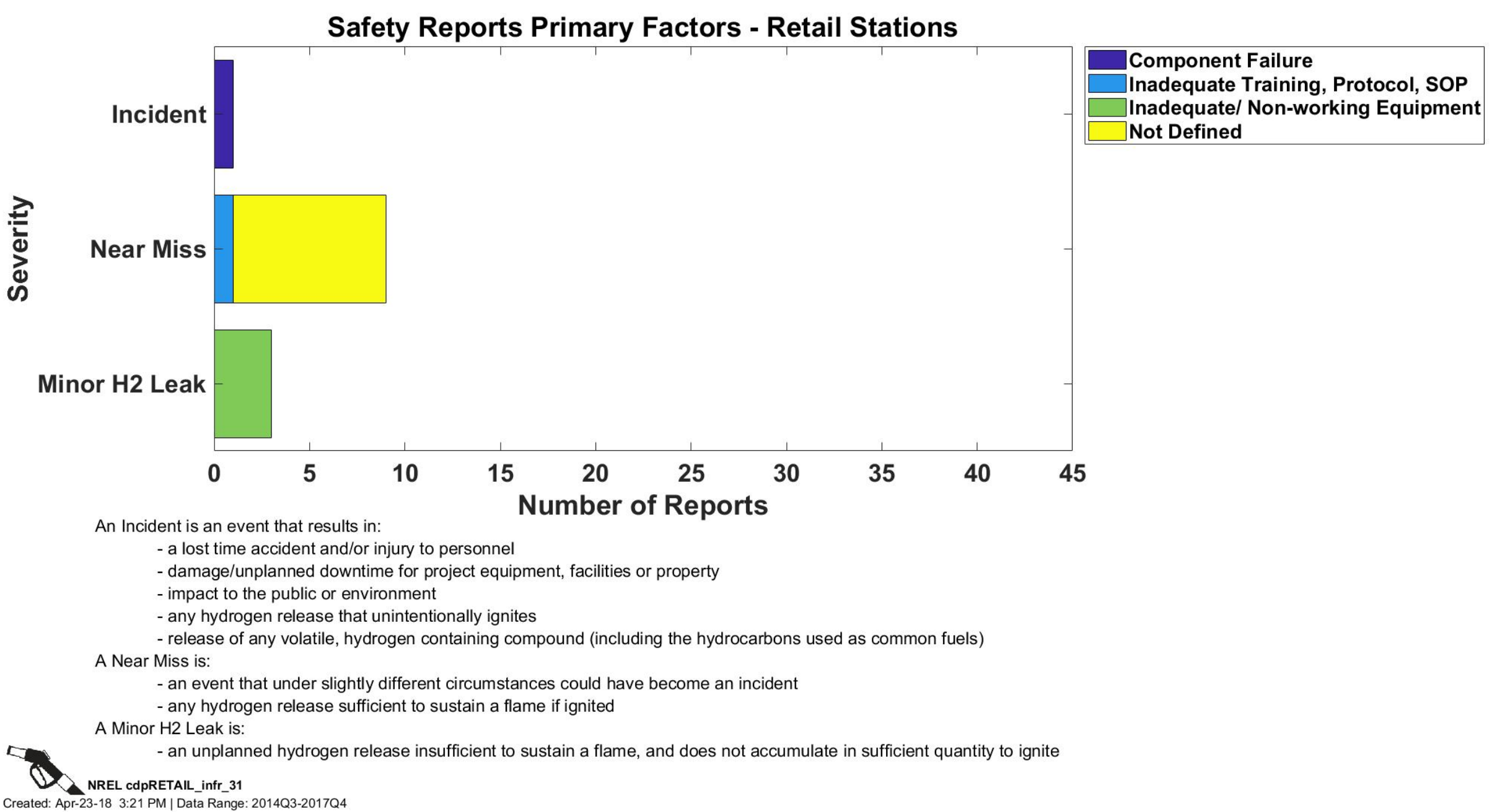




\section{Safety Reports by Equipment Involved}

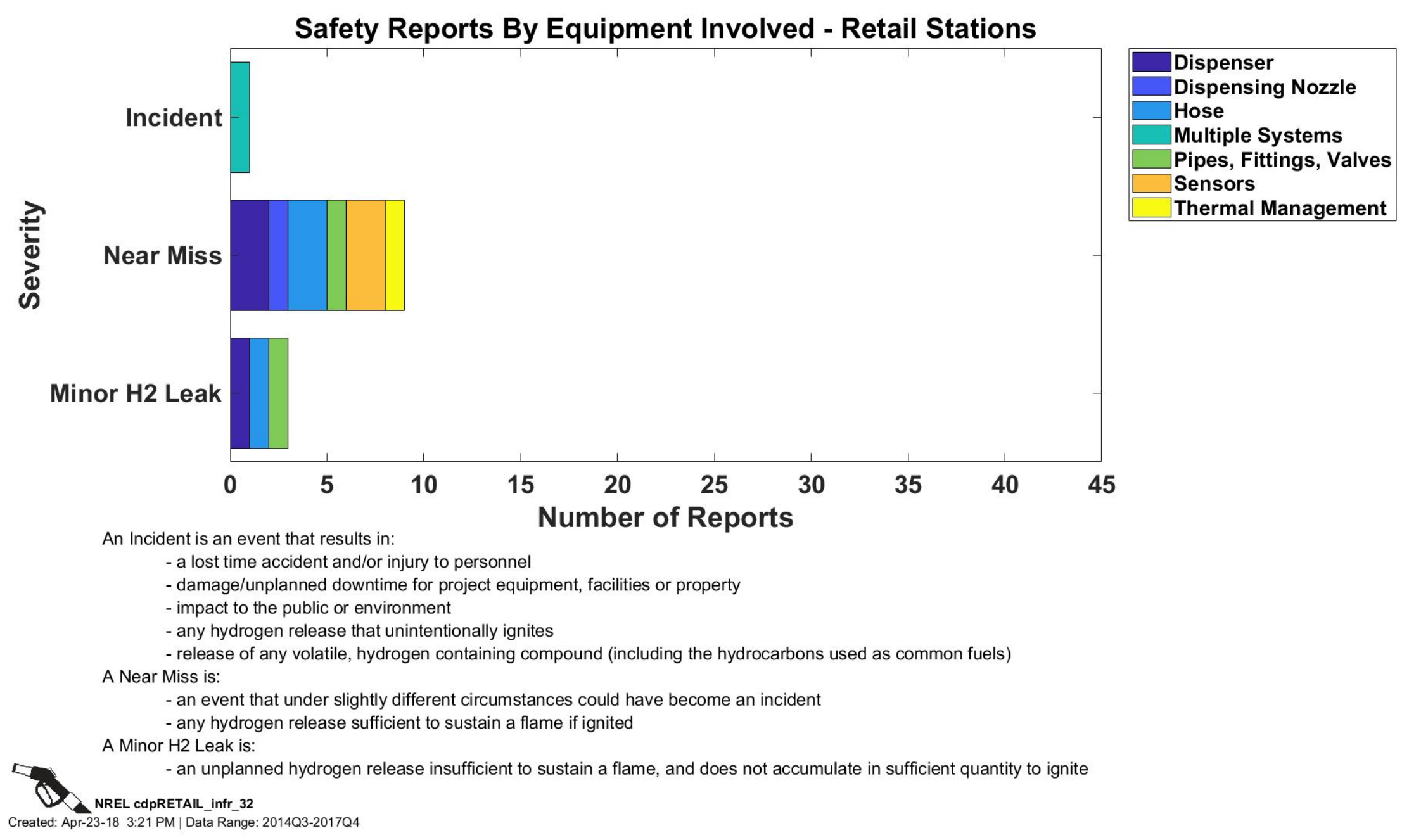




\section{Safety Reports by Quarter}

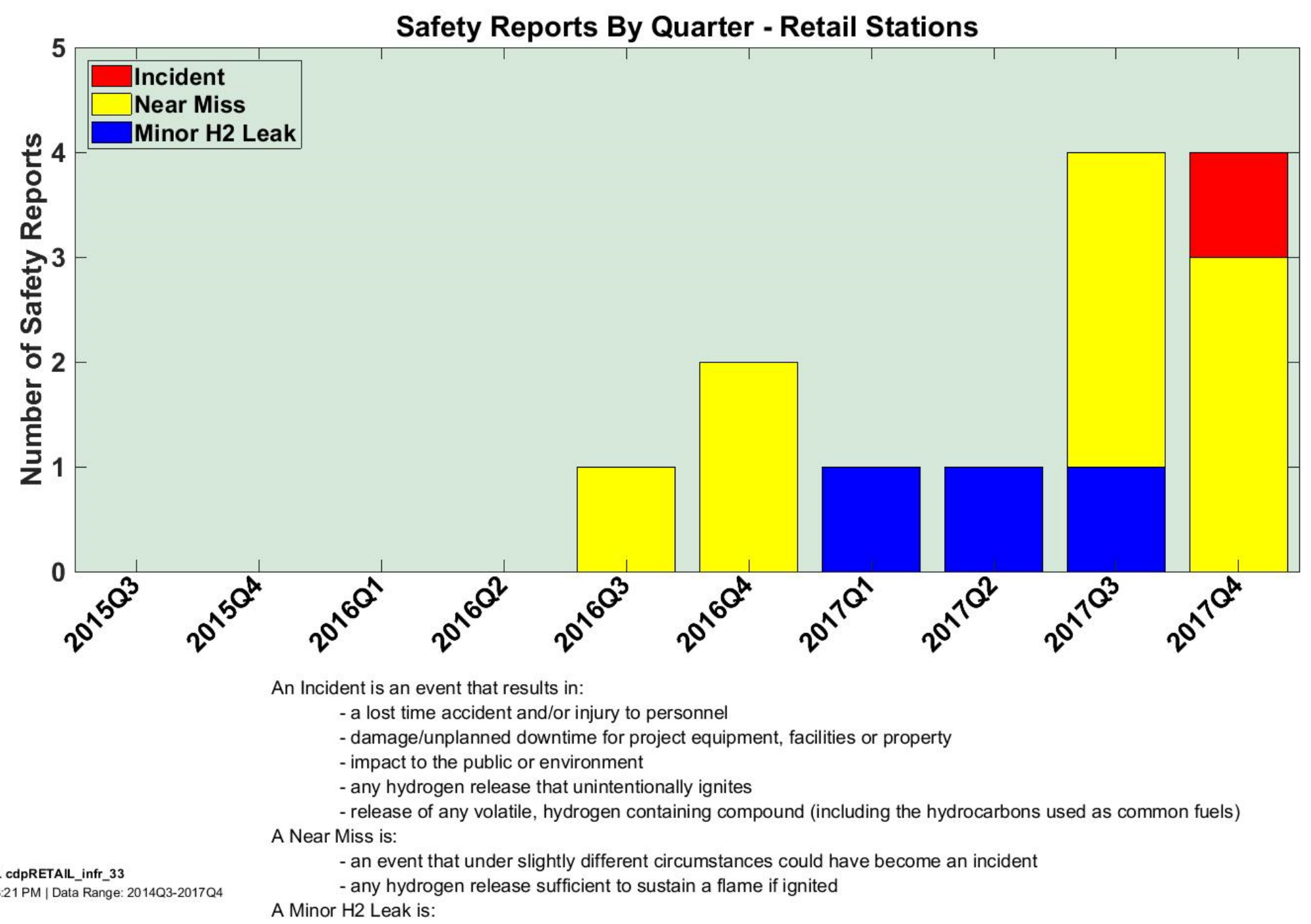




\section{Safety Reports by Event Description}

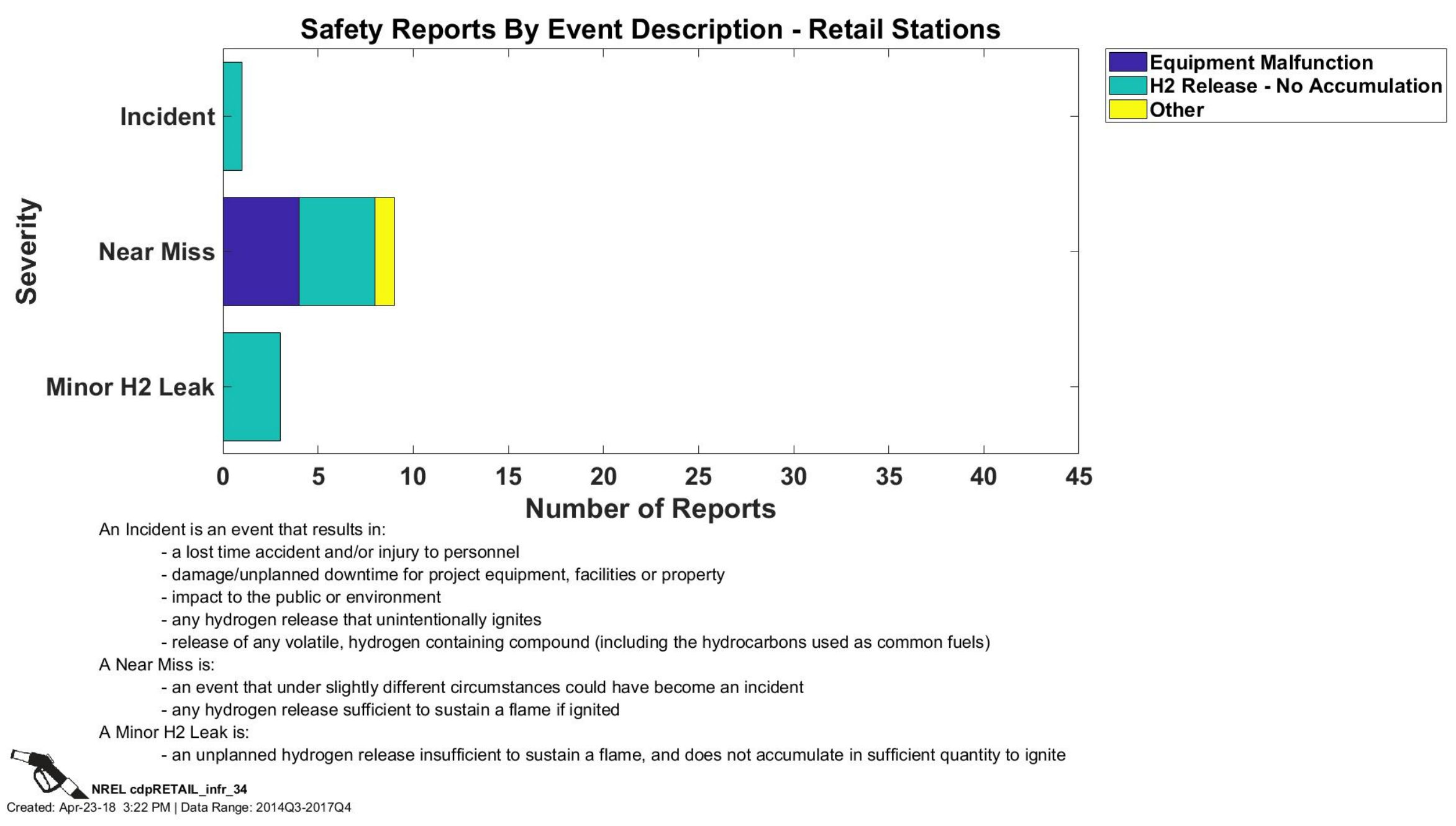




\section{Mean Fills per Hydrogen Leak}

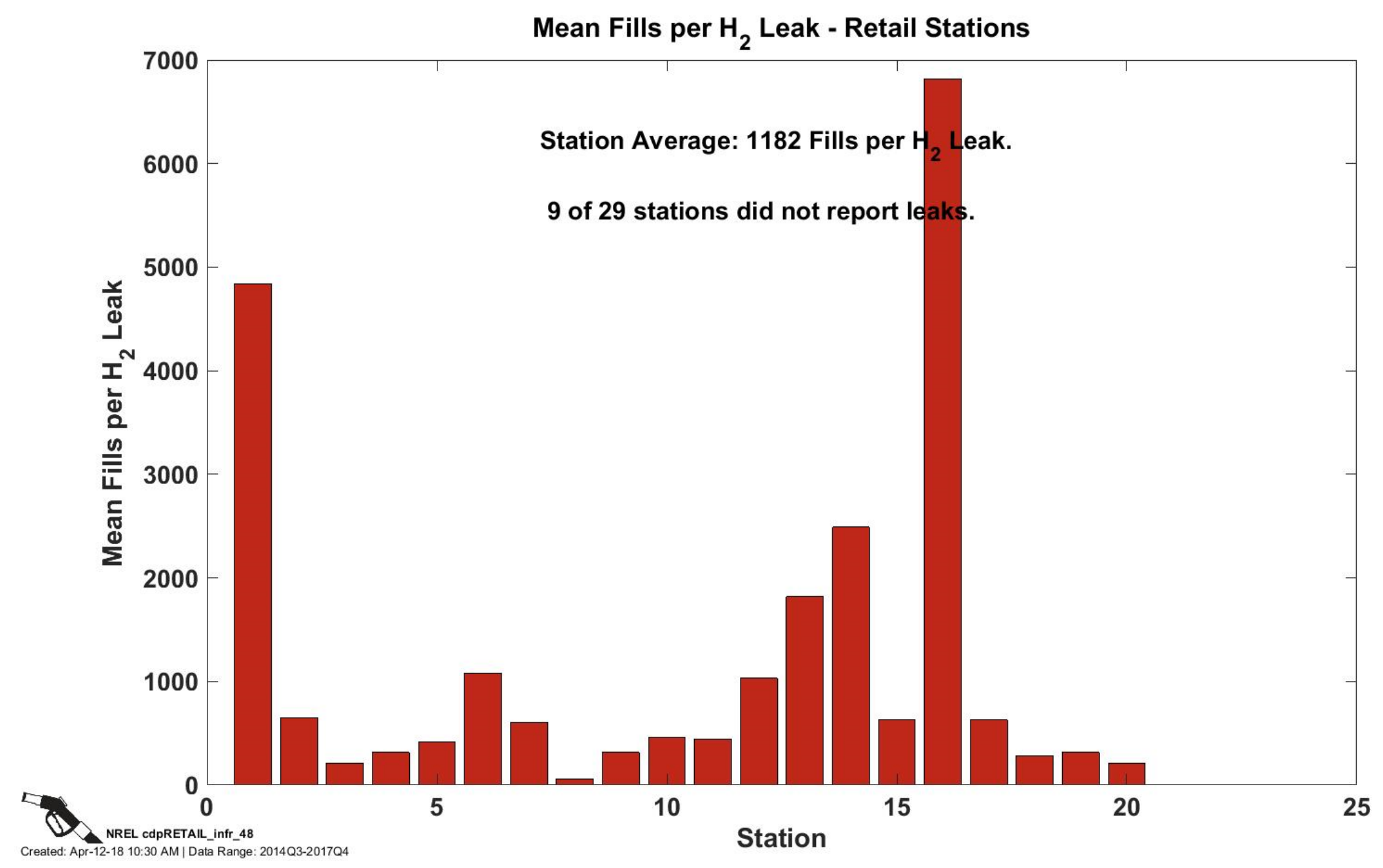




\section{Mean Hydrogen Dispensed per Hydrogen Leak}

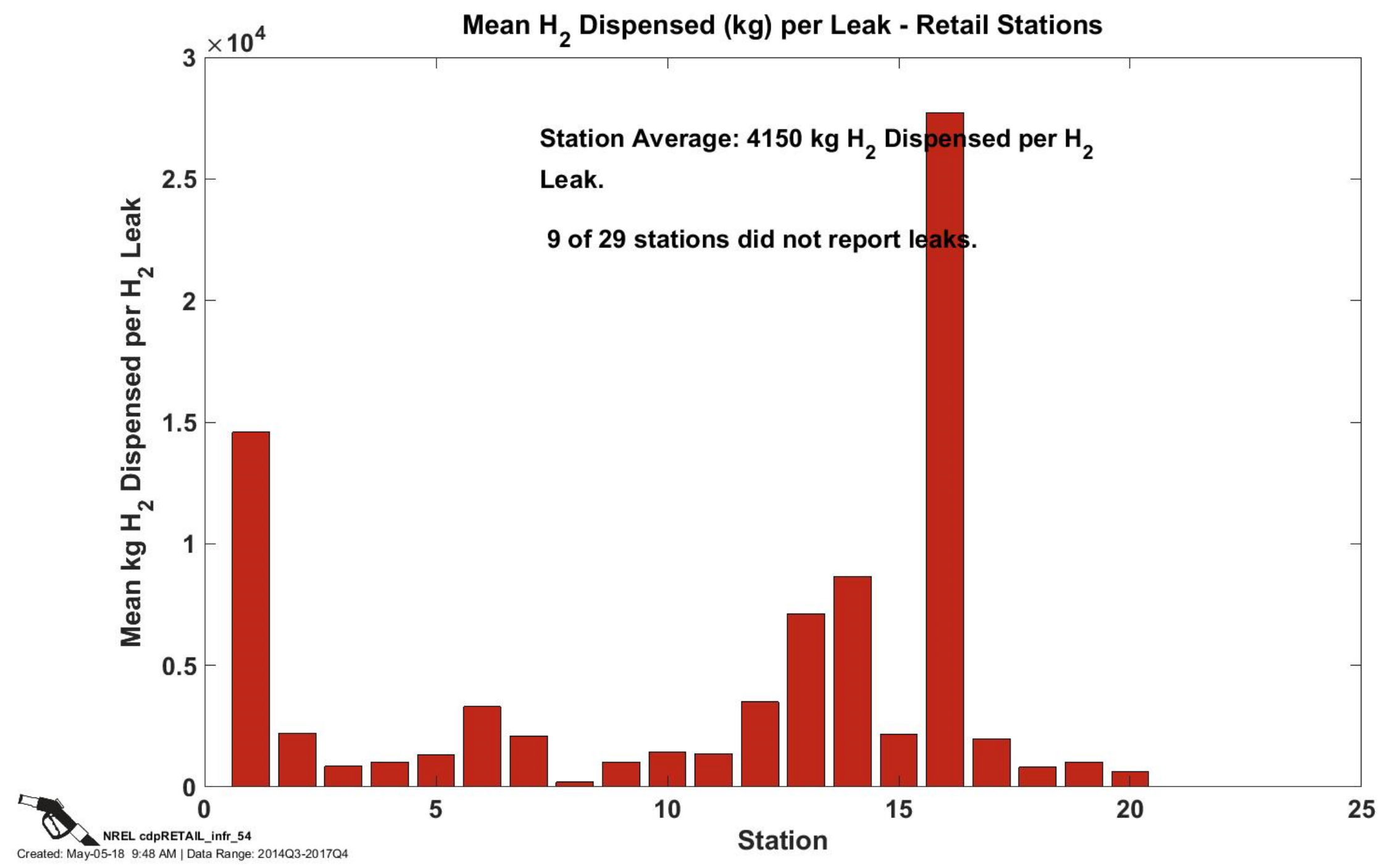


Maintenance and Reliability

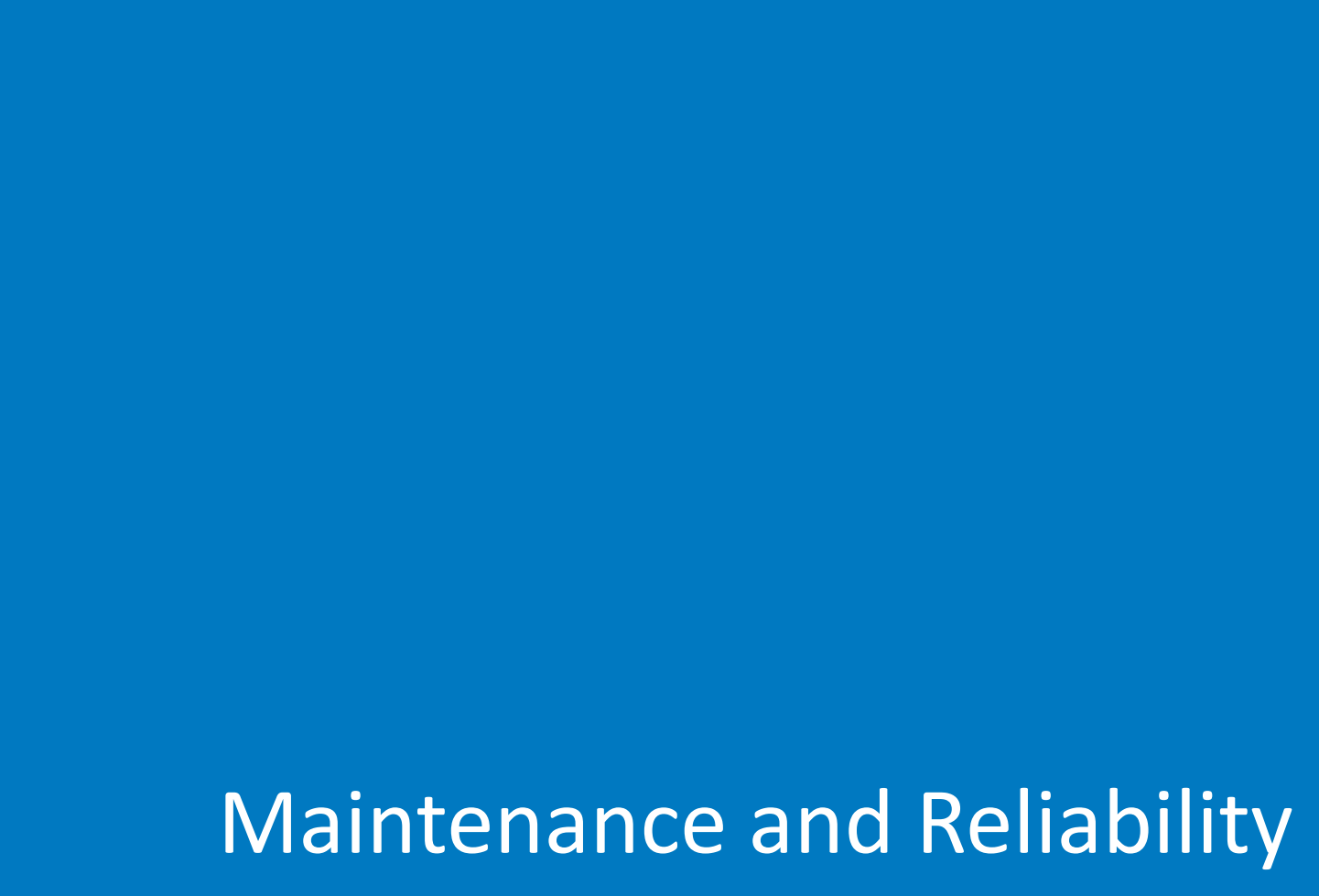




\section{CDP-INFR-21 \\ Maintenance by Equipment Type}

\section{Maintenance by Equipment Type - Retail Stations}
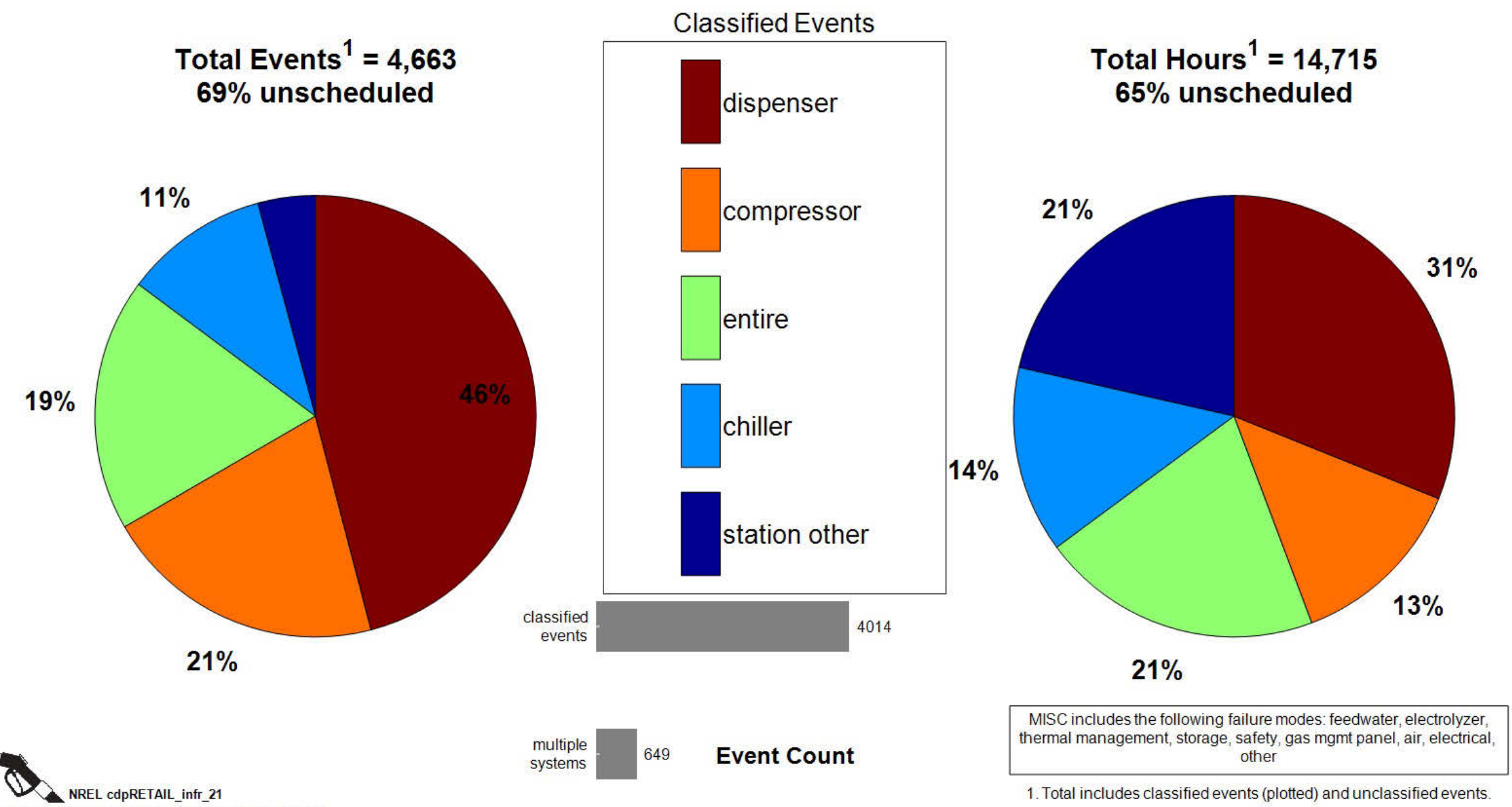

NREL cdpRETAIL_infr_ 21 


\section{CDP-INFR-22 \\ Maintenance Labor Hours per Event}

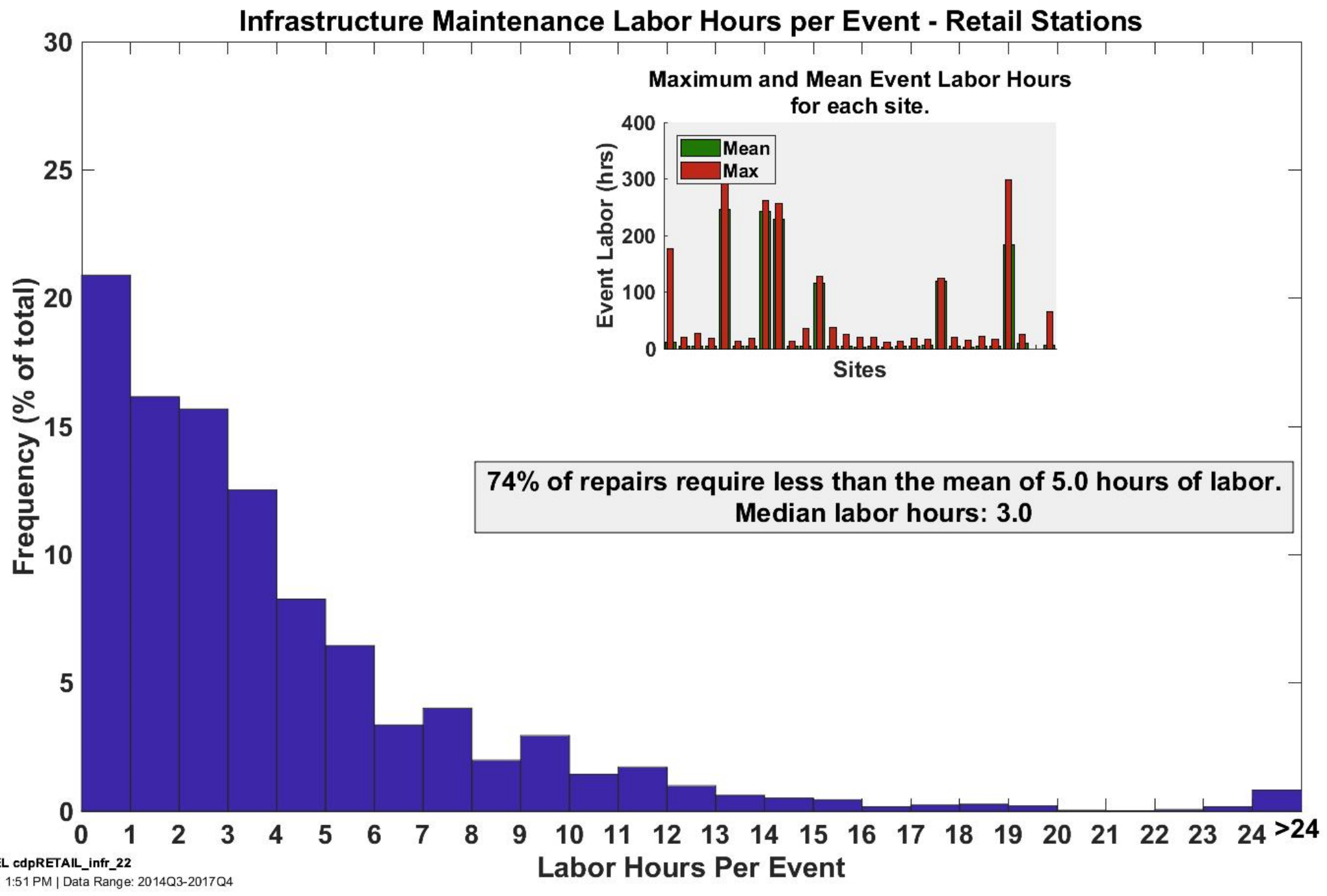




\section{CDP-INFR-23}

\section{Equipment Category Repair Time}

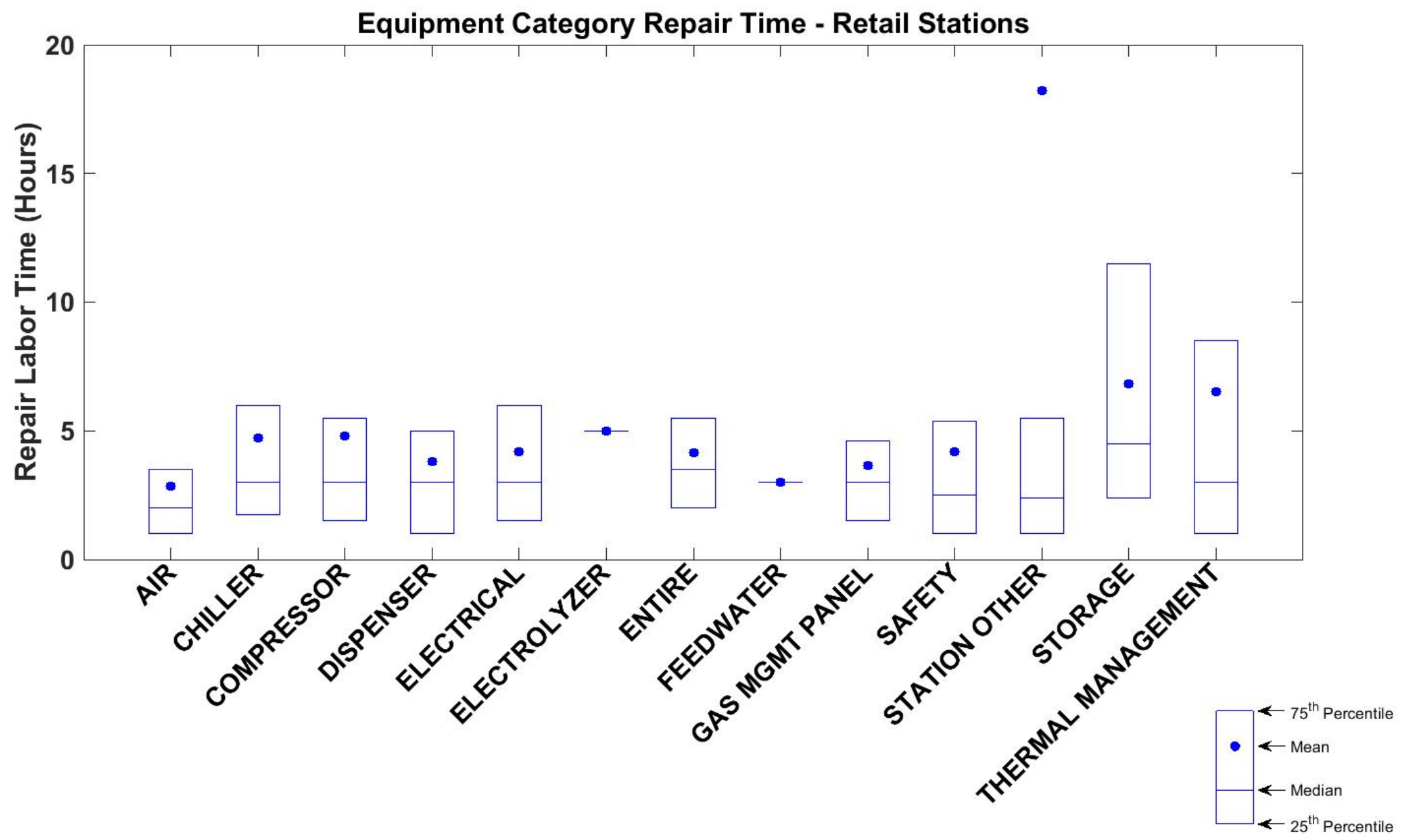

NREL cdpRETAIL_infr_23 


\section{Failure Modes for Top Equipment Categories}

\section{Failure Modes for Top Equipment Categories - Retail Stations}
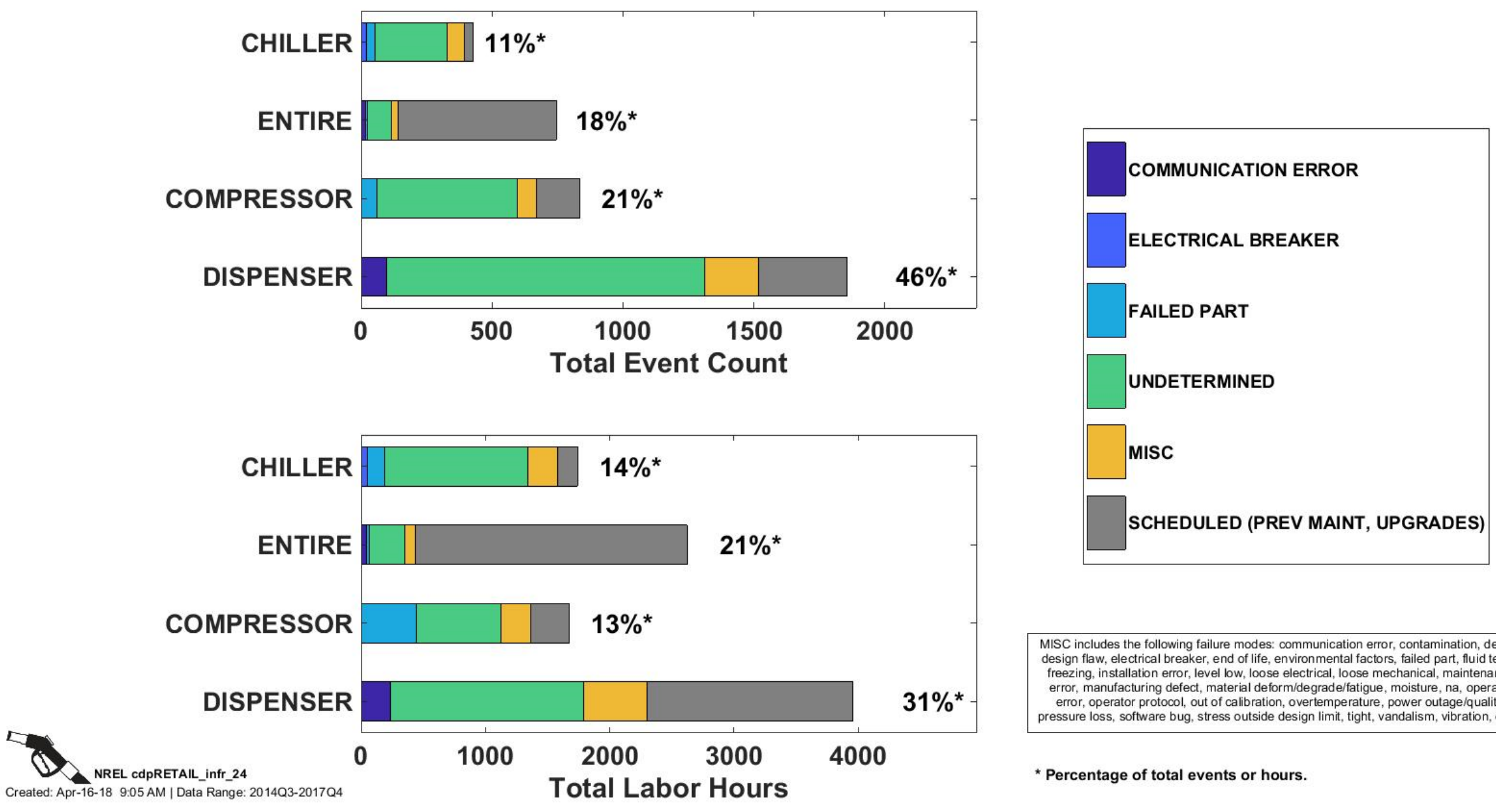


\section{CDP-INFR-26}

\section{Compressor Monthly Maintenance}
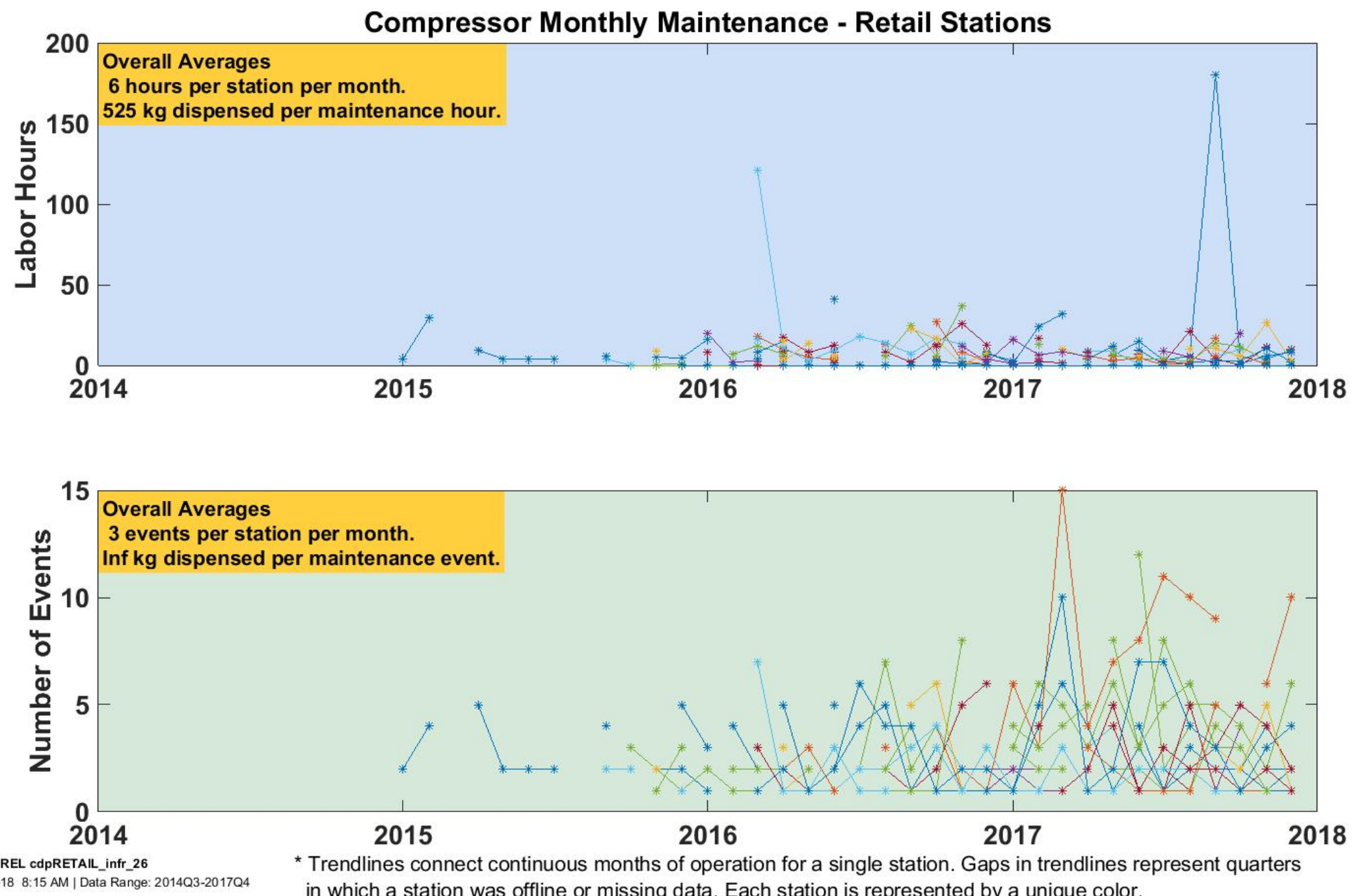


\section{Maintenance Labor Hours by Quarter}

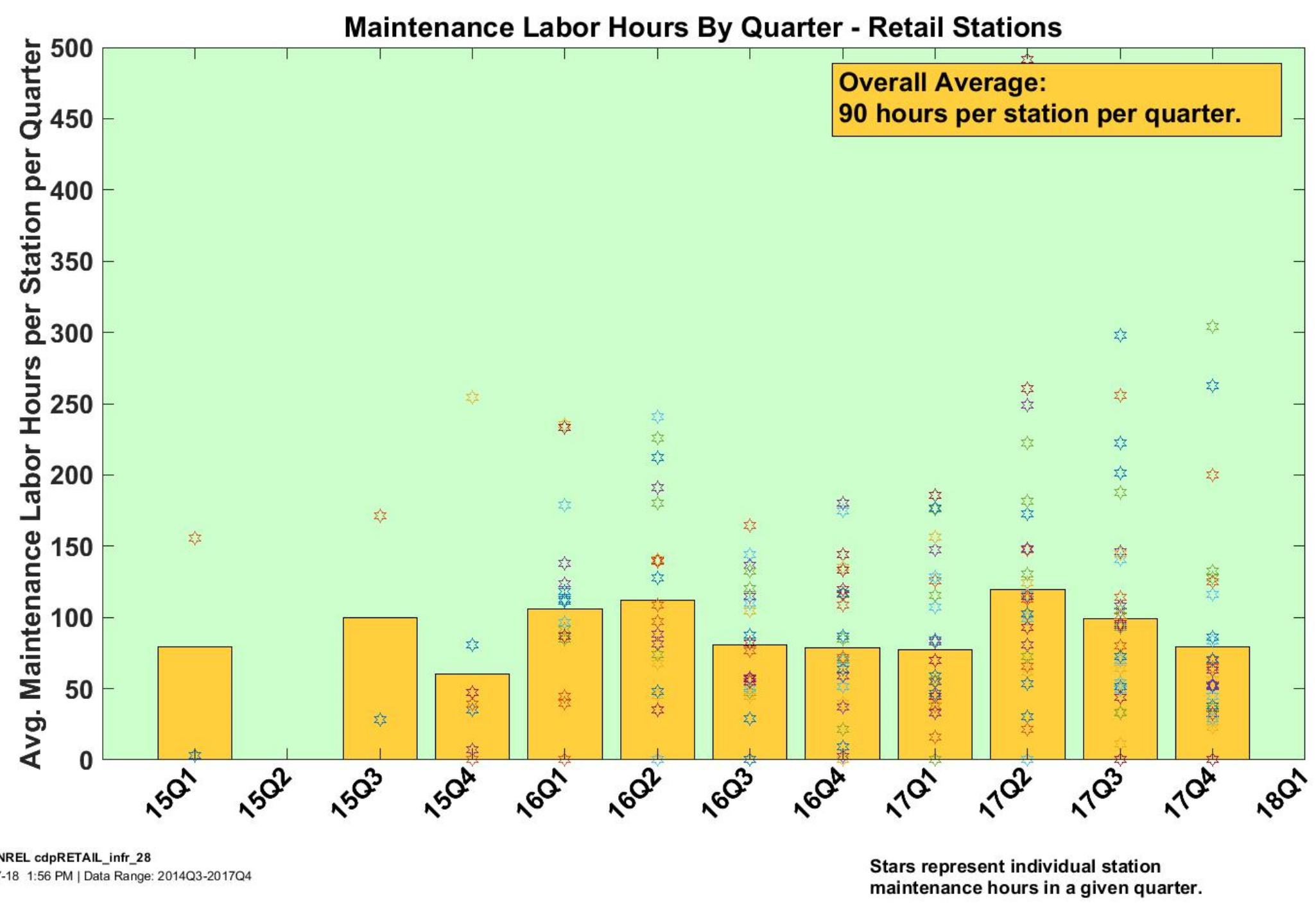




\section{CDP-INFR-30 \\ Maintenance Costs Over Time}

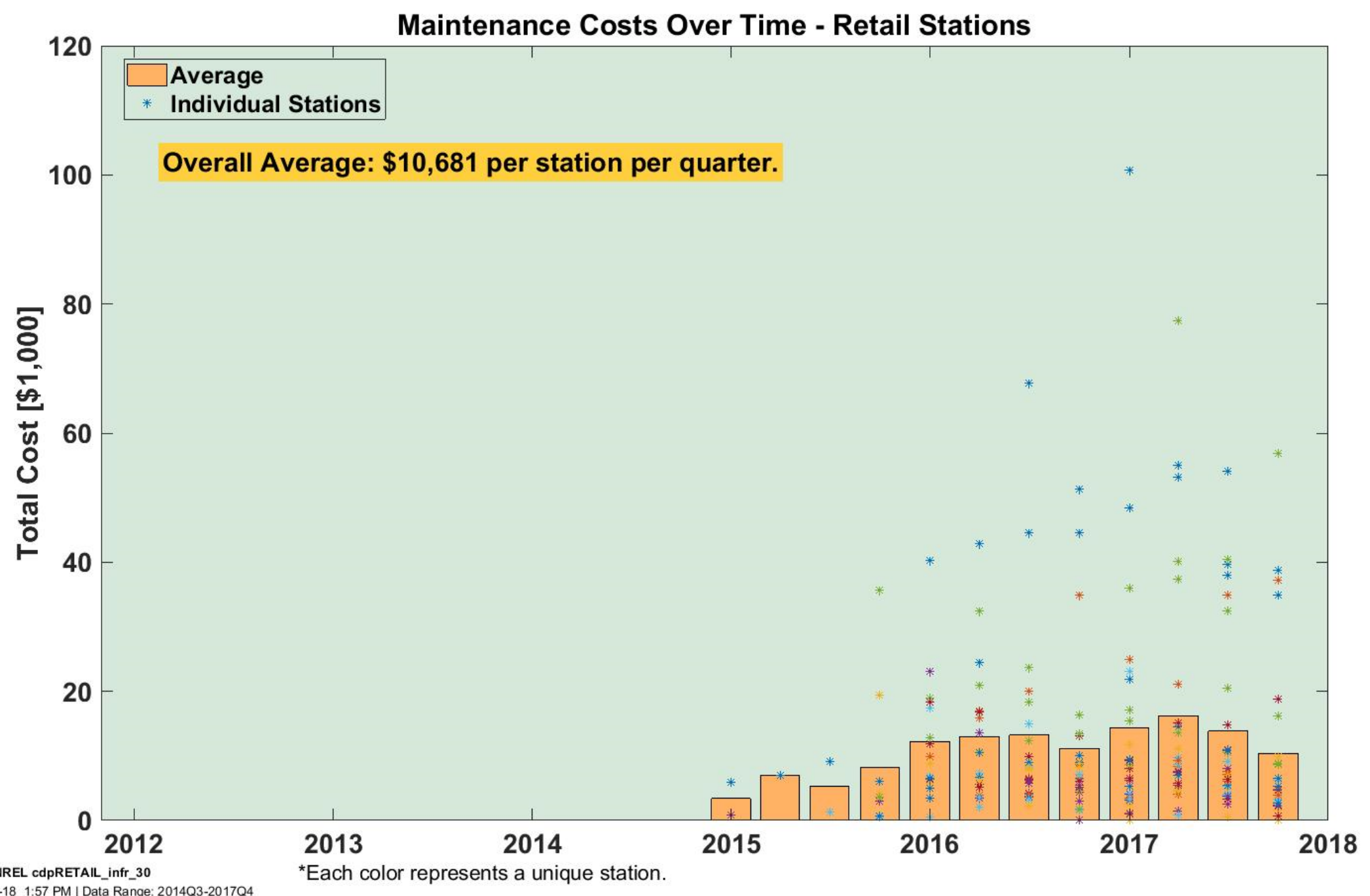




\section{CDP-INFR-49}

\section{Mean Fills Between Failures}

\section{Mean Fills Between Failures - Retail Stations}

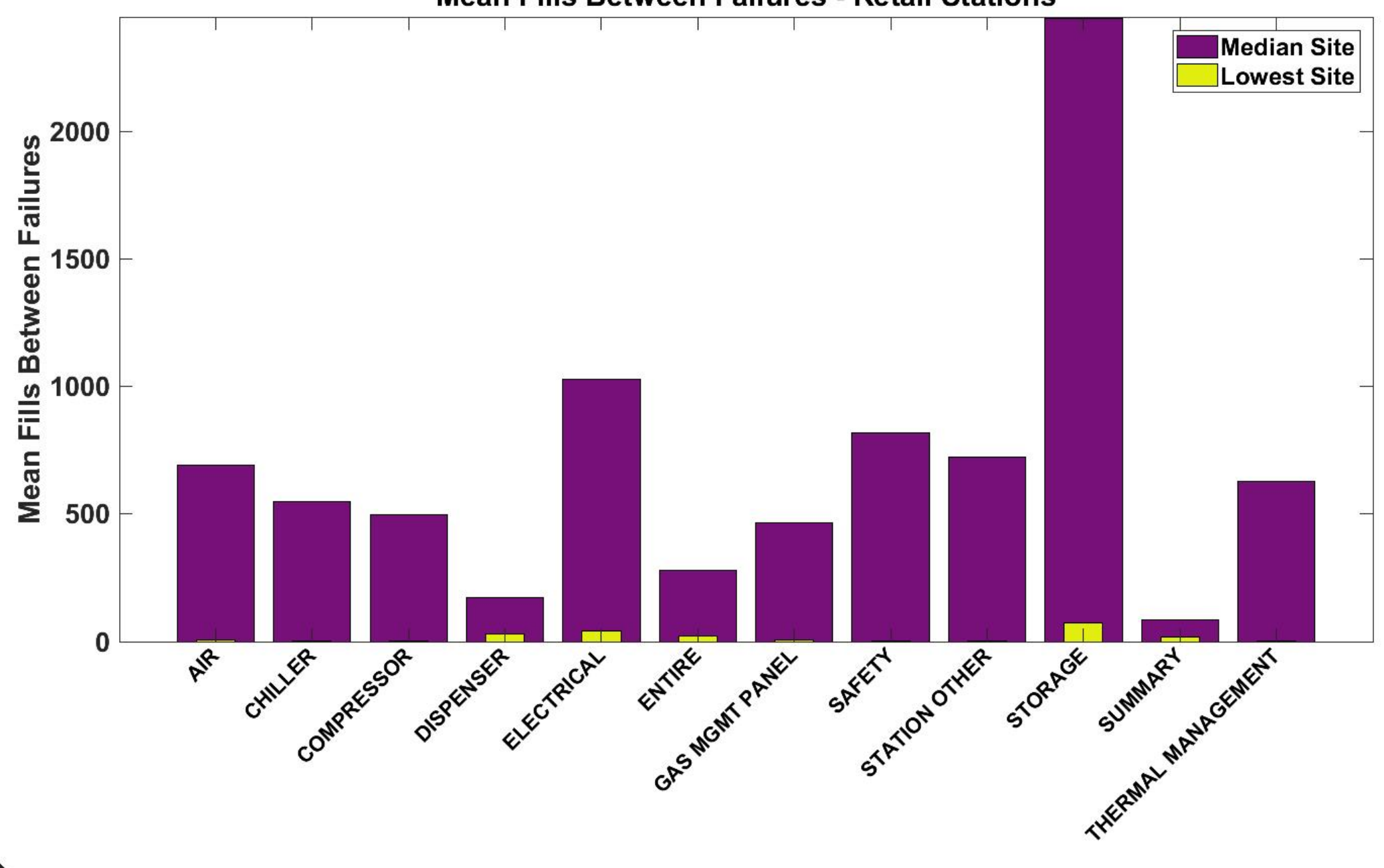




\section{CDP-INFR-50 Reliability Growth by Fills}

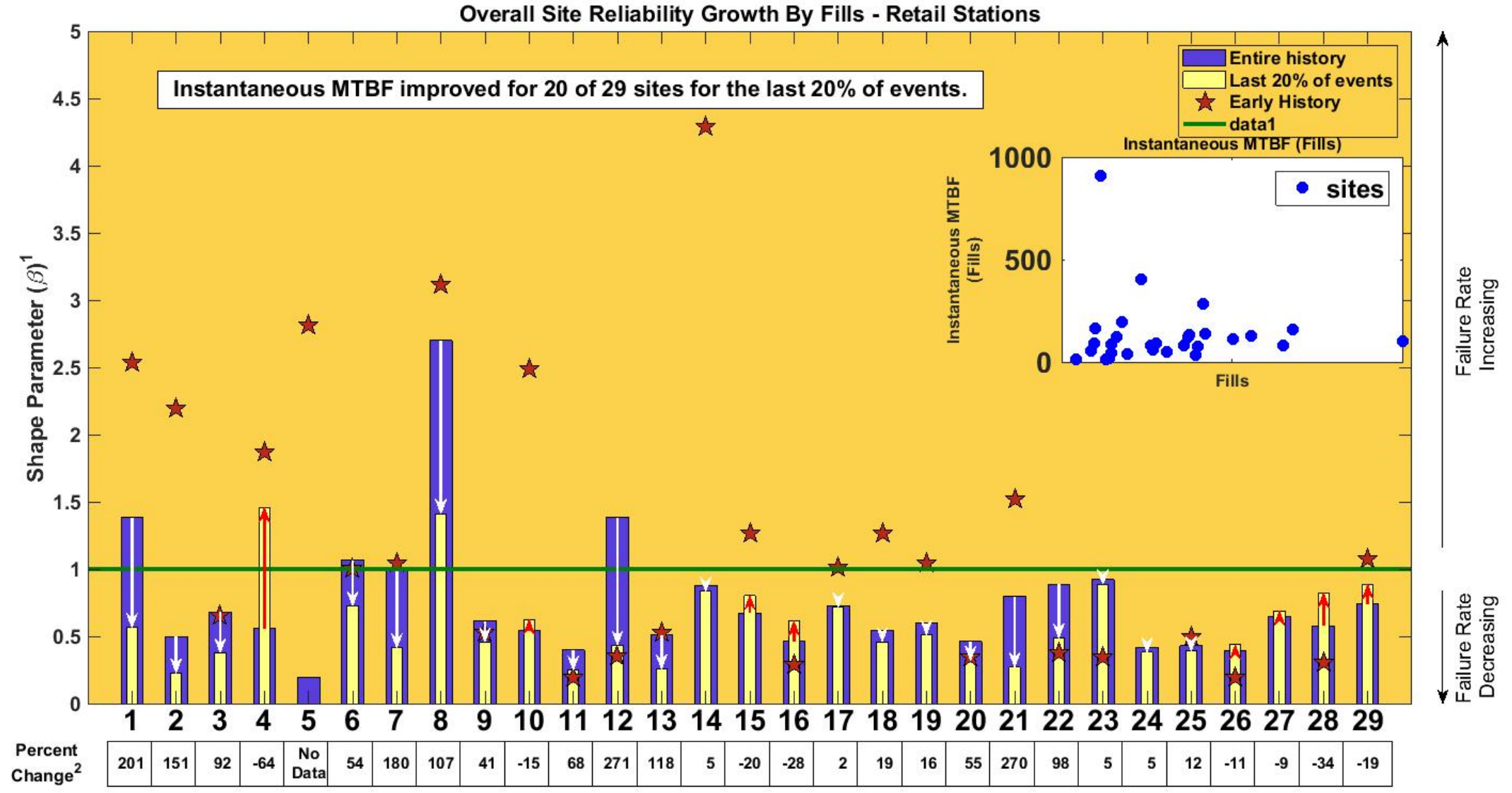

Sites sorted by Increasing Age Fills

1. IEC 61164:2004(E)., Reliability Growth - Statistical Test and Evaluation Methods, IEC. 2004.

2. \% change in instantaneous mean Fills between failures 


\section{Mean Amount Dispensed Between Failures}

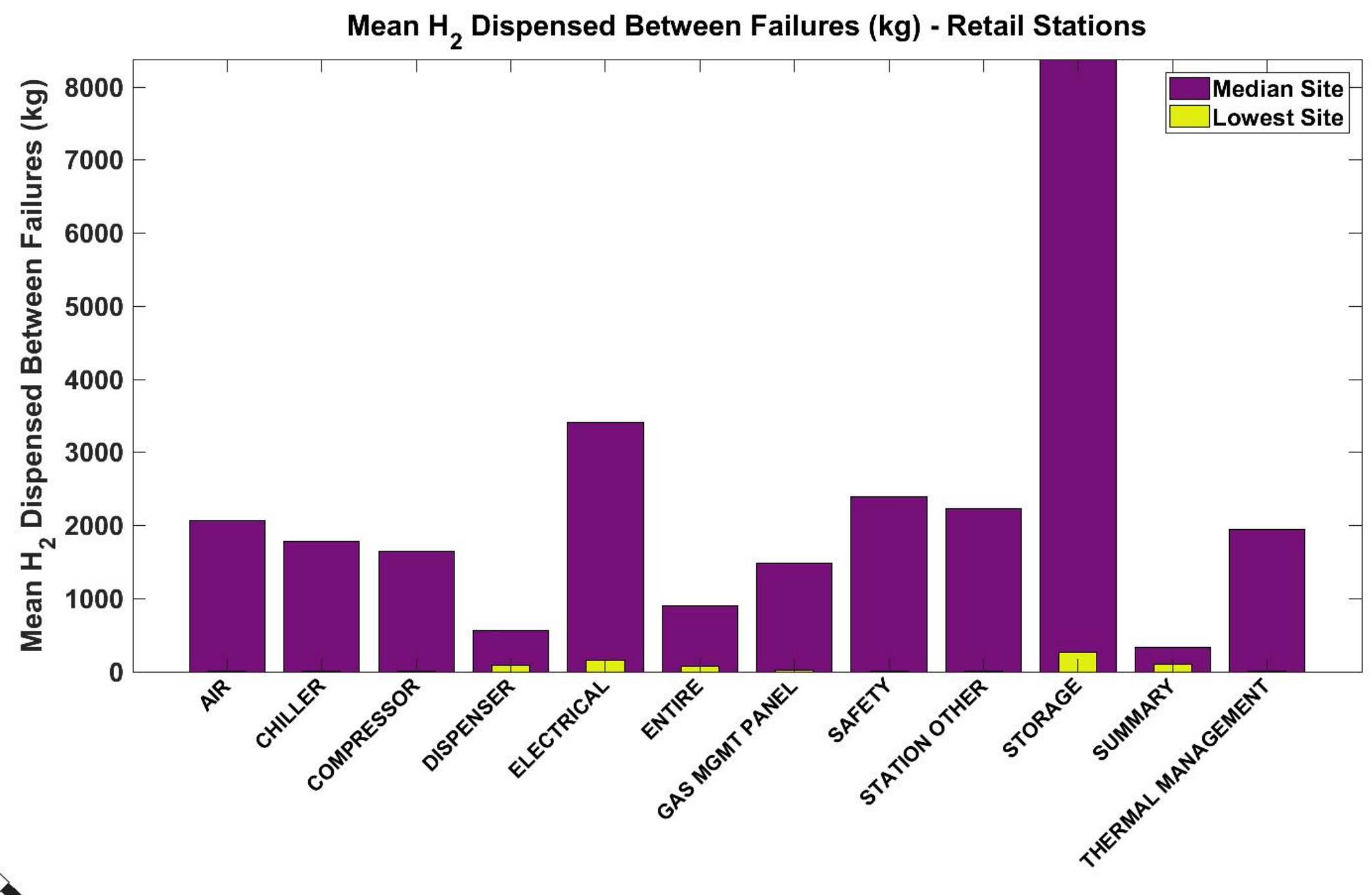




\section{CDP-INFR-52}

\section{Reliability Growth by Amount Dispensed}

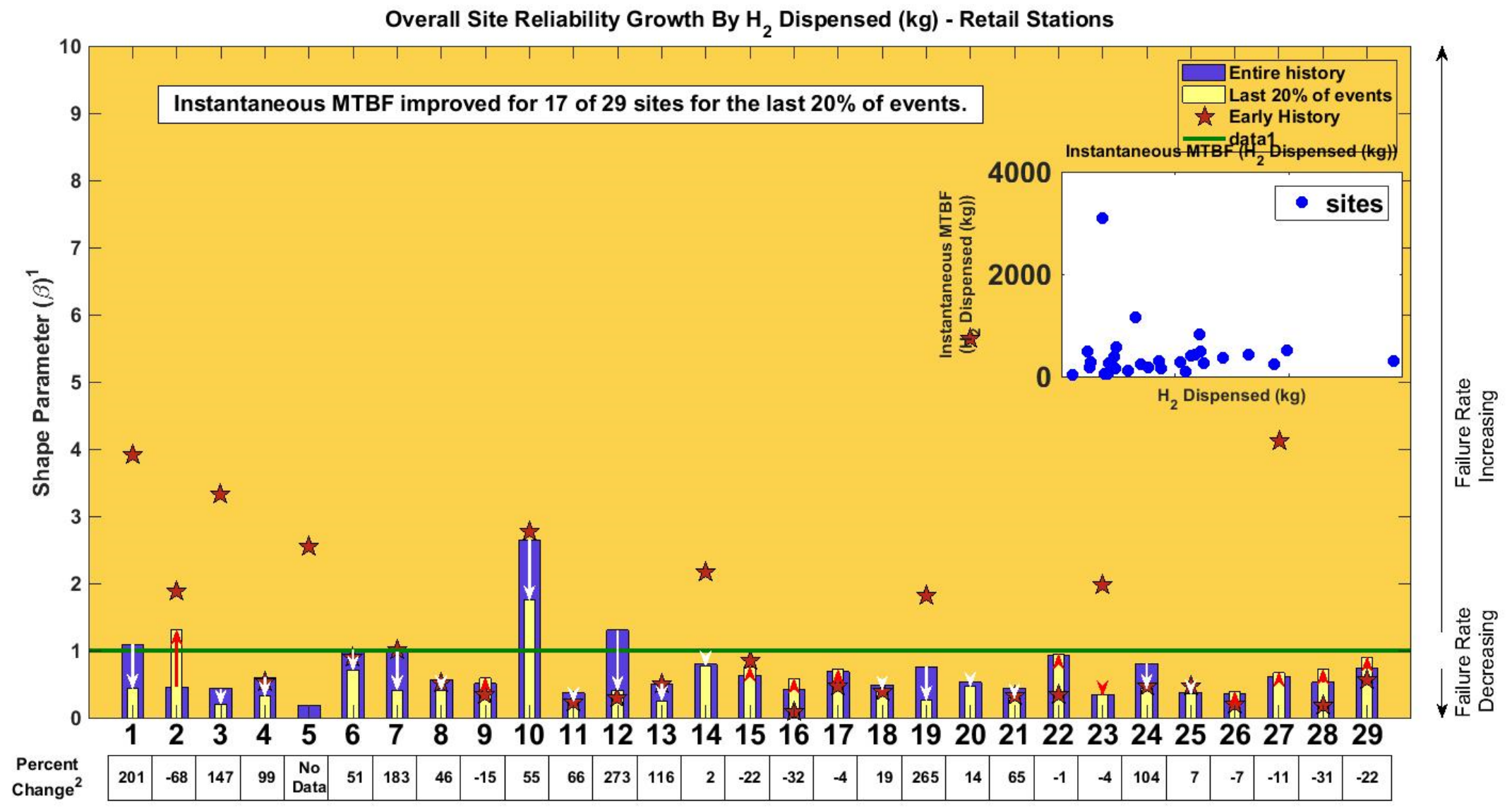

Sites sorted by Increasing Age

$\mathrm{H}_{2}$ Dispensed (kg)

1. IEC 61164:2004(E)., Reliability Growth - Statistical Test and Evaluation Methods, IEC. 2004.

2. \% change in instantaneous mean $\mathrm{H}_{2}$ Dispensed $(\mathrm{kg})$ between failures 


\section{CDP-INFR-59 Historical Failure Rate by Fills}

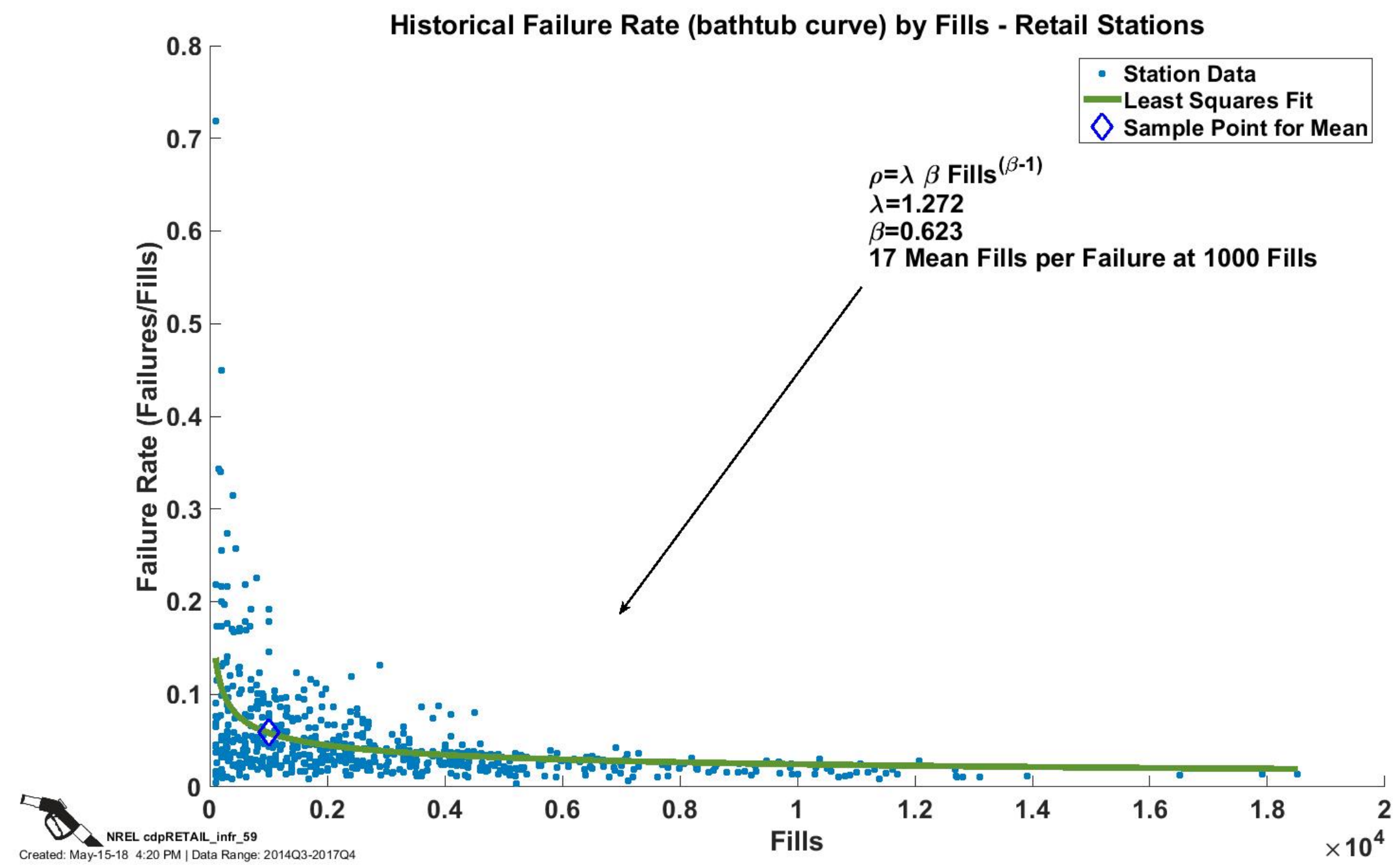




\section{Historical Failure Rate by Amount Dispensed}

CDP-INFR-60

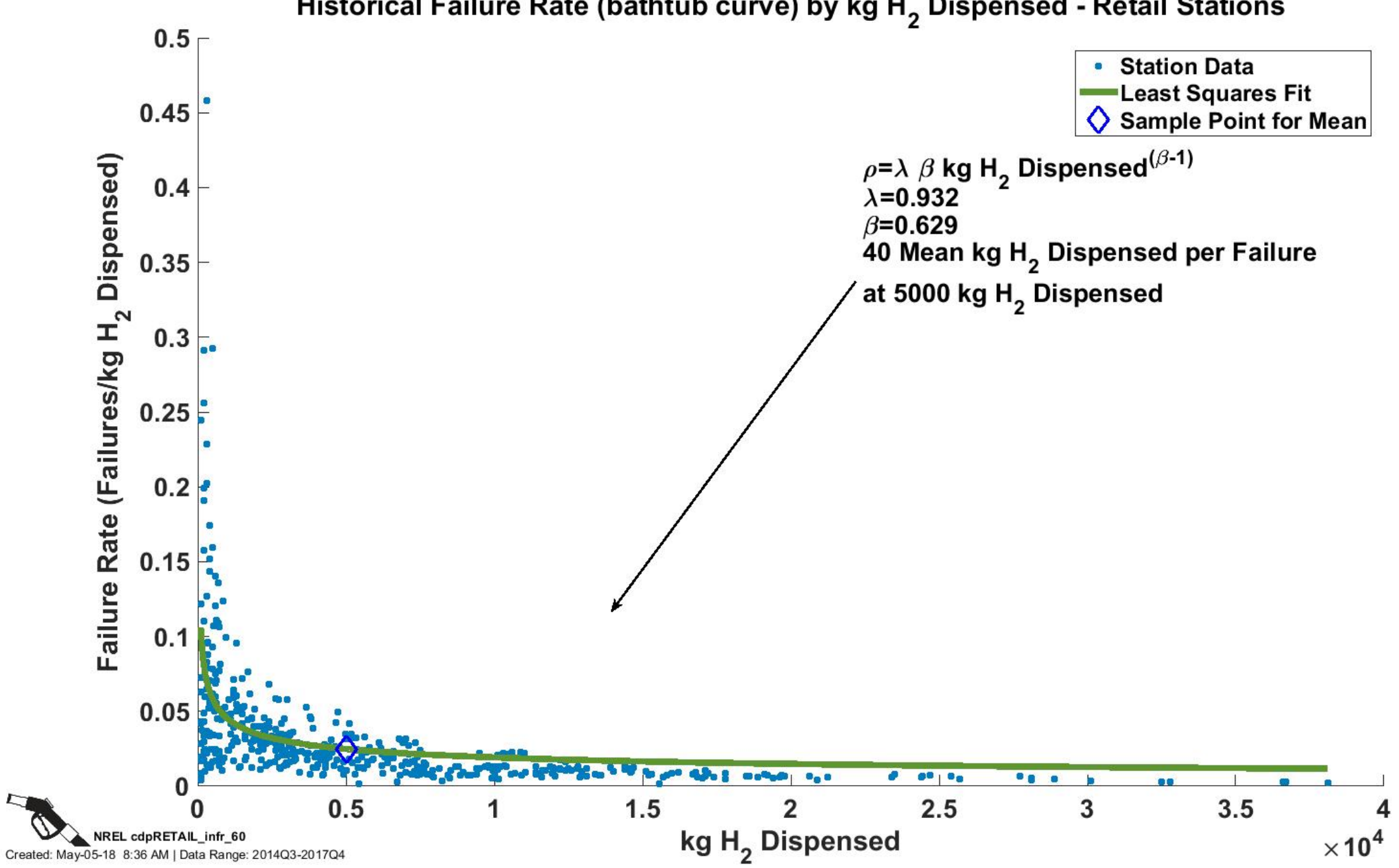




\section{CDP-INFR-62 \\ Mean Fills per Hydrogen Leak Over Time}

Mean Fills per $\mathrm{H}_{2}$ Leak Over Time - Retail Stations

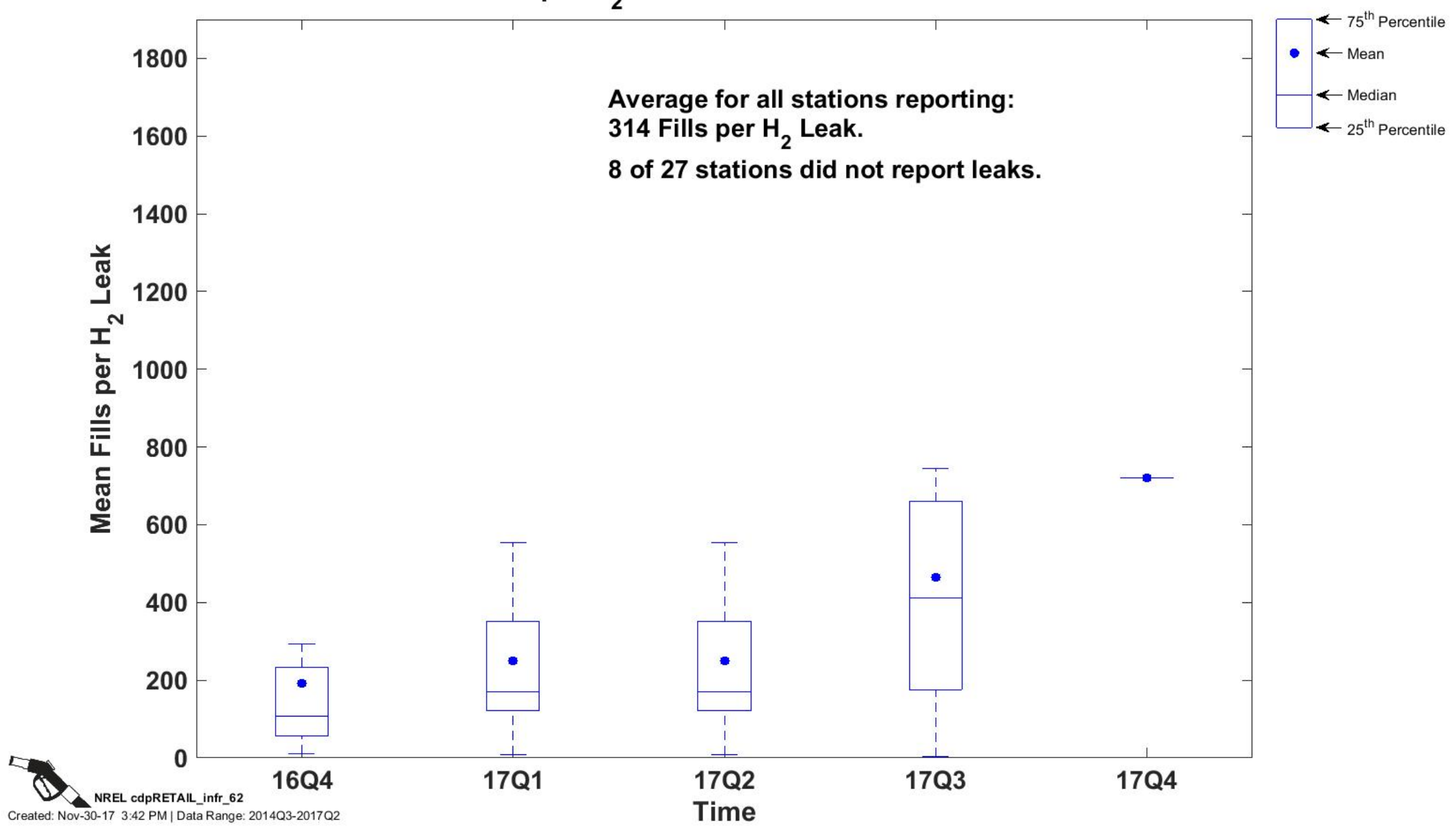




\section{CDP-INFR-63}

\section{Mean Hydrogen Dispensed per Leak Over Time}

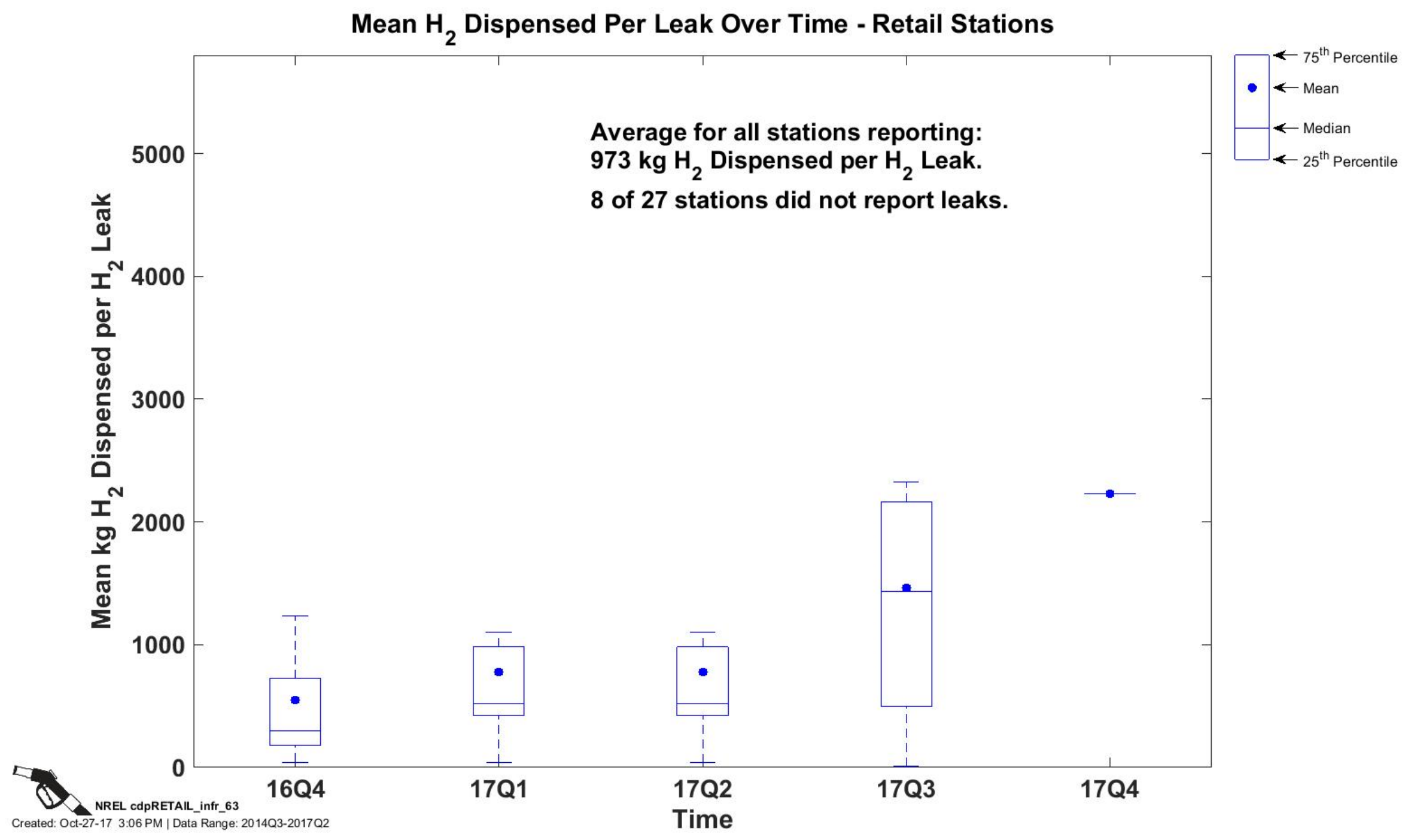




\section{CDP-INFR-64}

\section{Maintenance Causes and Effects: Safety (Software)}

Maintenance Causes and Effects - Retail Stations

Subsystem: SAFETY

Component: SOFTWARE

Preventative Maintenance accounted for $85 \%$ of all events.

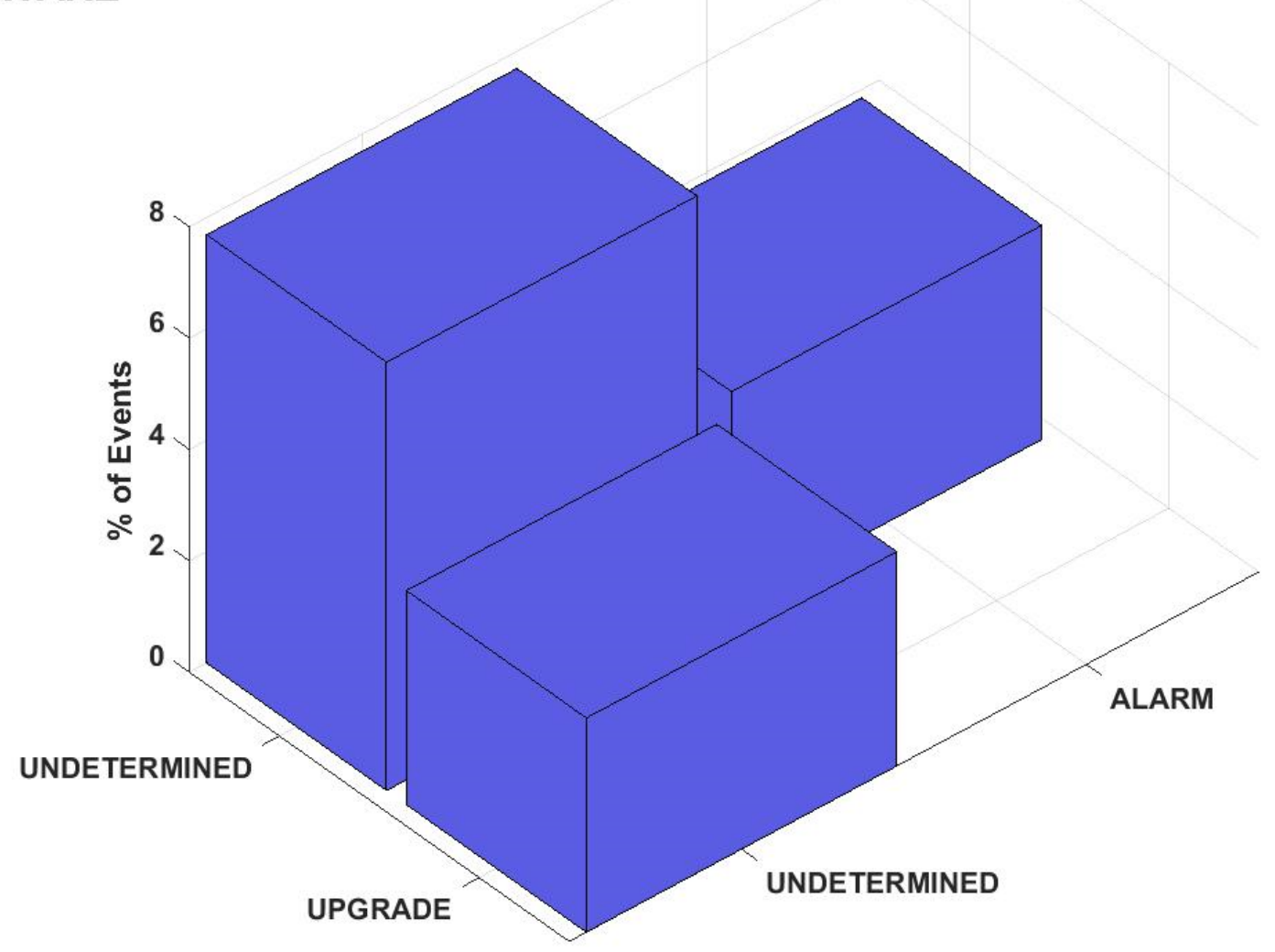

Causes

Effects

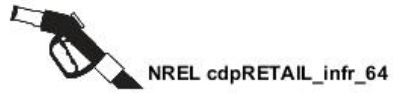

Created: May-05-18 8:18 AM | Data Range: 2014Q3-2017Q4 


\section{CDP-INFR-65}

\section{Maintenance Causes and Effects: Safety (Sensor)}

Maintenance Causes and Effects - Retail Stations

Subsystem: SAFETY

Component: SENSOR

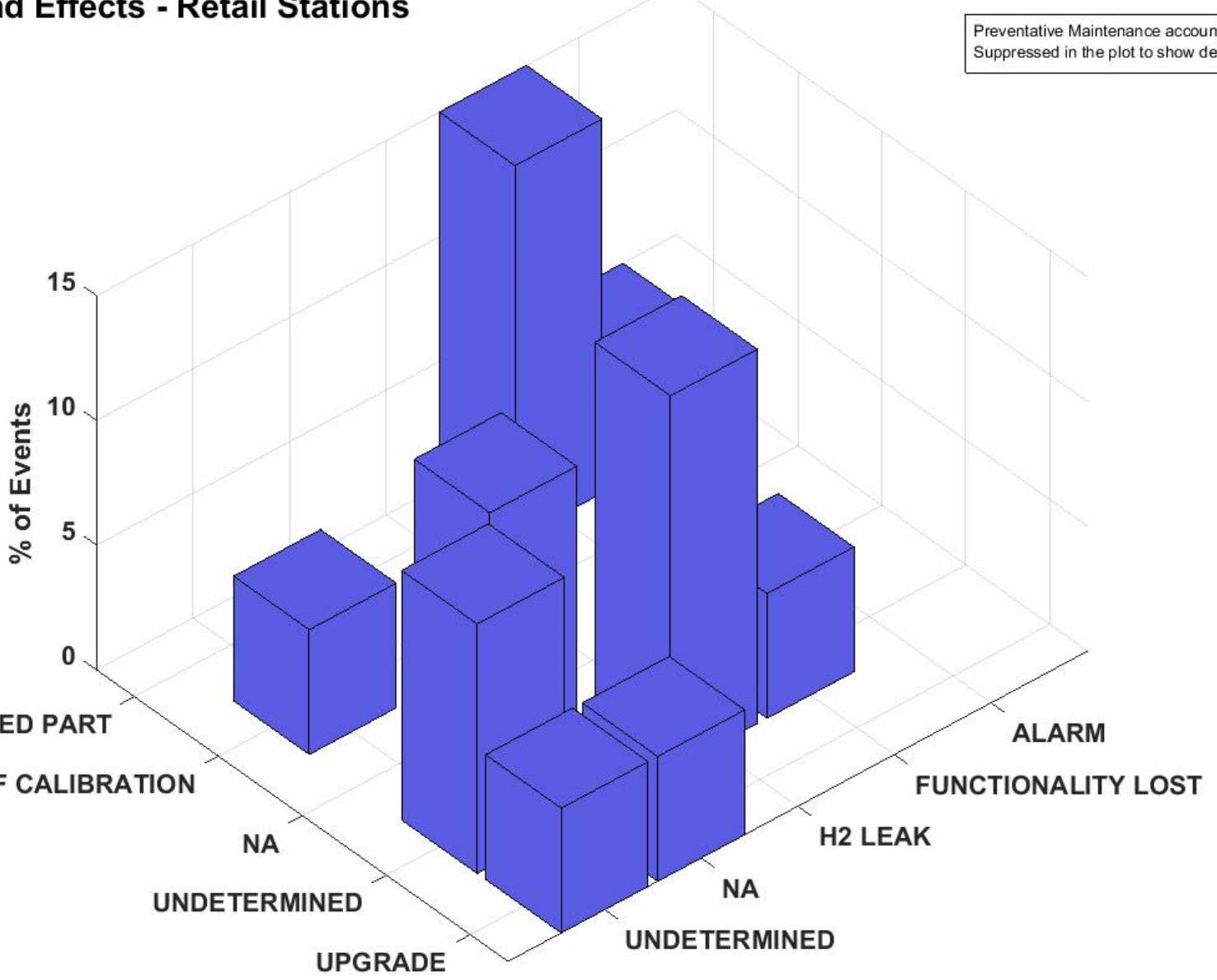

Causes

UPGRADE

Effects 


\title{
CDP-INFR-66
}

\section{Maintenance Causes and Effects: Safety (Entire)}

Maintenance Causes and Effects

Subsystem: SAFETY

Component: ENTIRE

\author{
$\sqrt{2}$
}

12

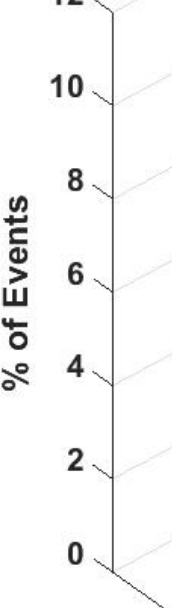

2.

10

UPGRADE

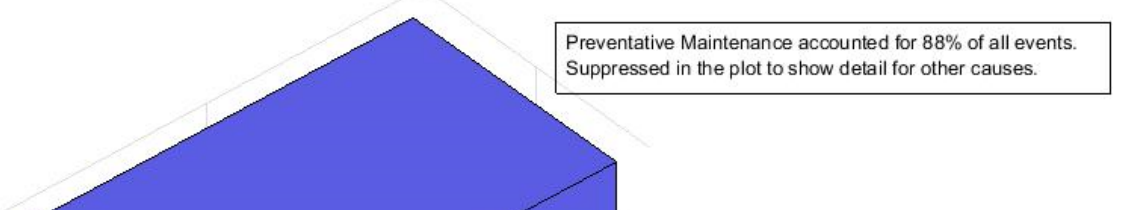

Causes

Effects

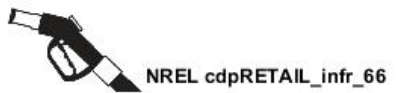

Created: May-05-18 8:07 AM | Data Range: 2014Q3-2017Q4 


\section{CDP-INFR-67}

\section{Maintenance Causes and Effects: Dispenser (Entire)}

Maintenance Causes and Effects - Retail Stations

Subsystem: DISPENSER

Component: ENTIRE

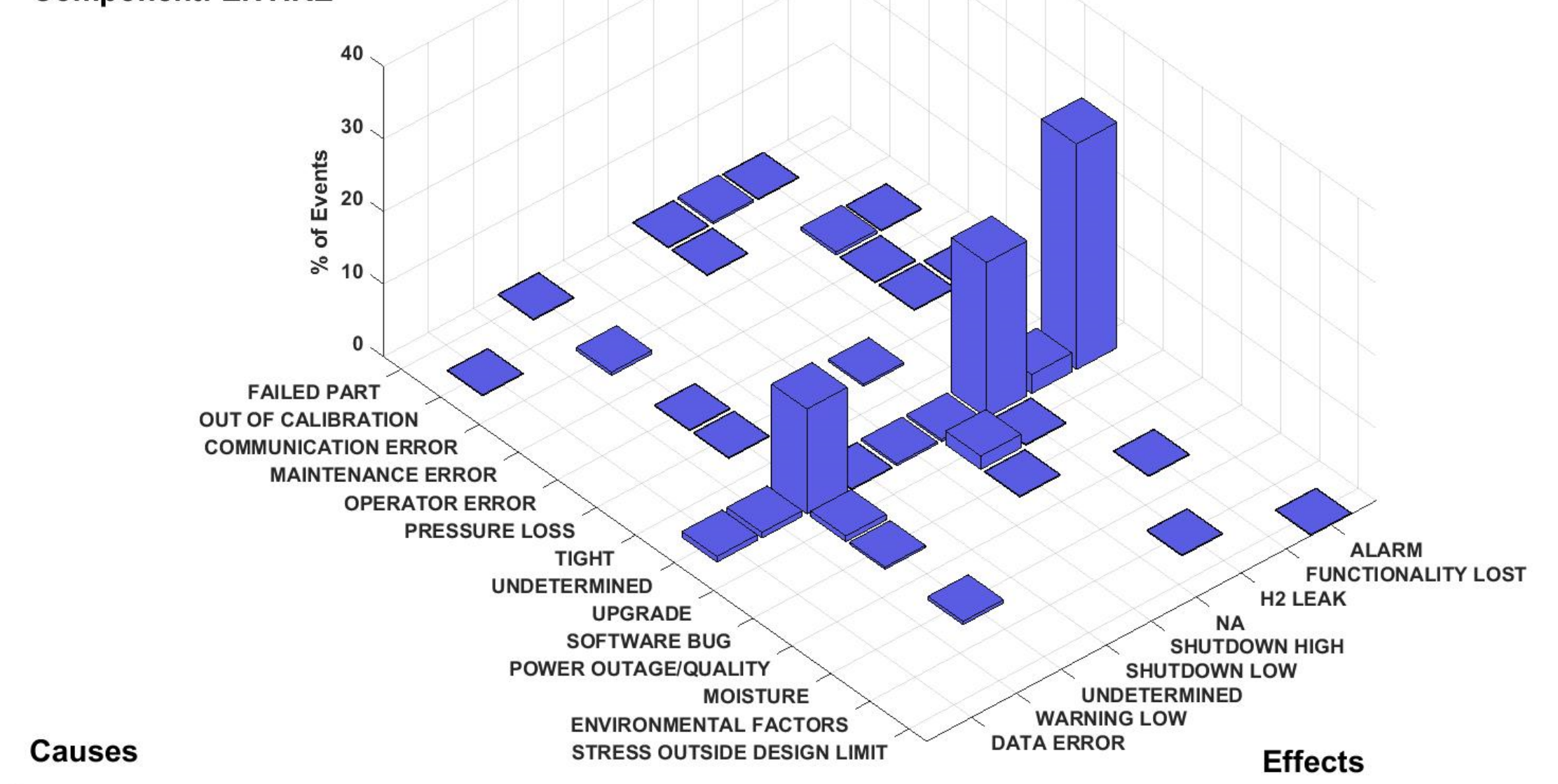




\section{CDP-INFR-68}

\section{Maintenance Causes and Effects: Dispenser (Fitting)}

Maintenance Causes and Effects - Retail Stations

Subsystem: DISPENSER

Component: FITTING

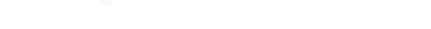

MATERIAL DEFORM/DEGRADE/FATIGUE

\section{Causes}

UNDETERMINED
Preventative Maintenance accounted for $0 \%$ of all events. Suppressed in the plot to show detail for other causes.

\section{(1). NREL_cdpRETAIL_infr_68}




\section{CDP-INFR-69}

\section{Maintenance Causes and Effects: Dispenser (Nozzle)}

\section{Maintenance Causes and Effects - Retail Stations}

Subsystem: DISPENSER

Component: NOZZLE

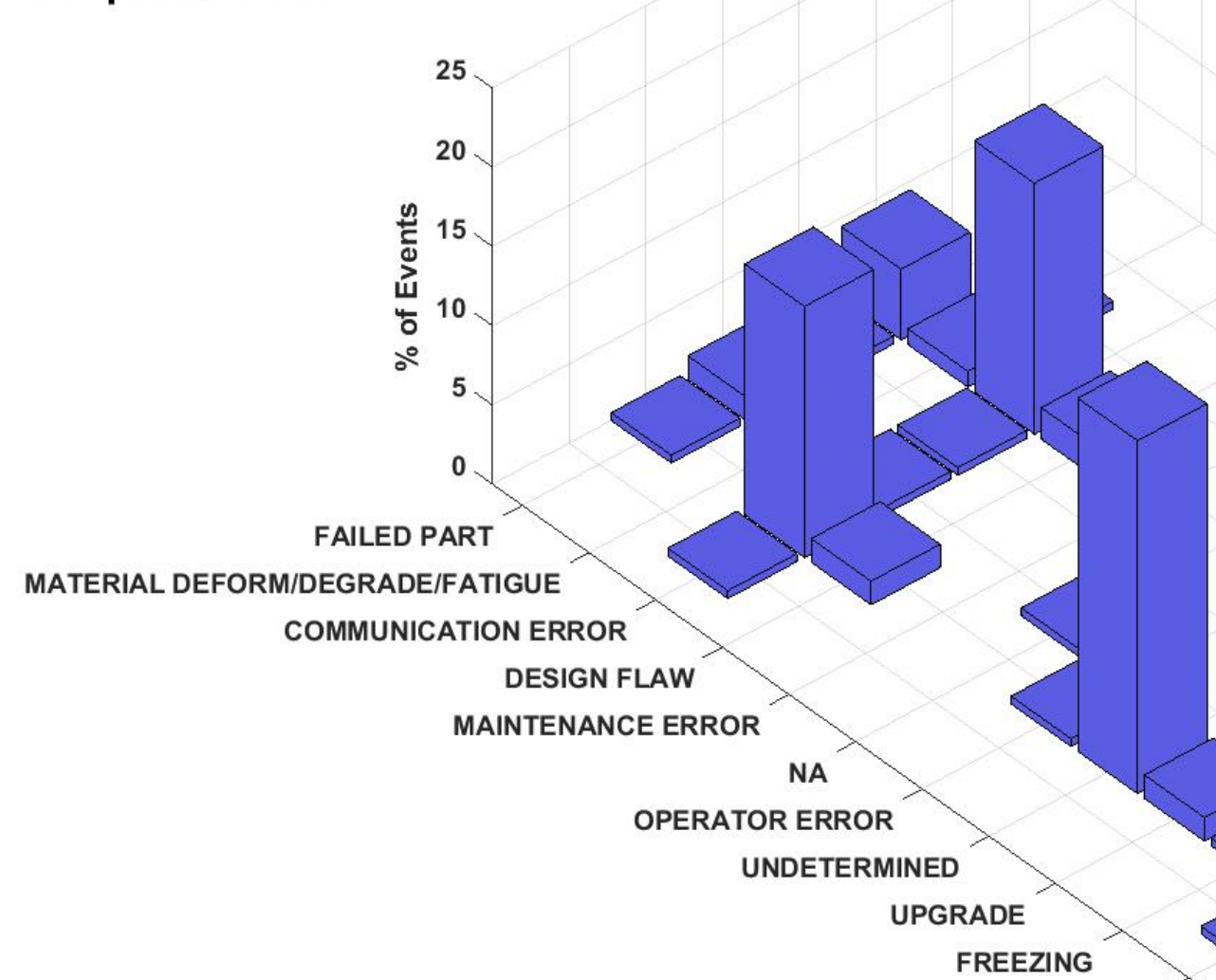

\section{Causes}

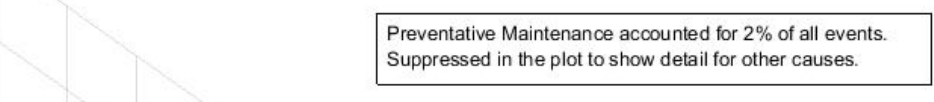




\section{CDP-INFR-70}

\section{Maintenance Causes and Effects: Compressor (Entire)}

Maintenance Causes and Effects - Retail Stations Subsystem: COMPRESSOR

Component: ENTIRE

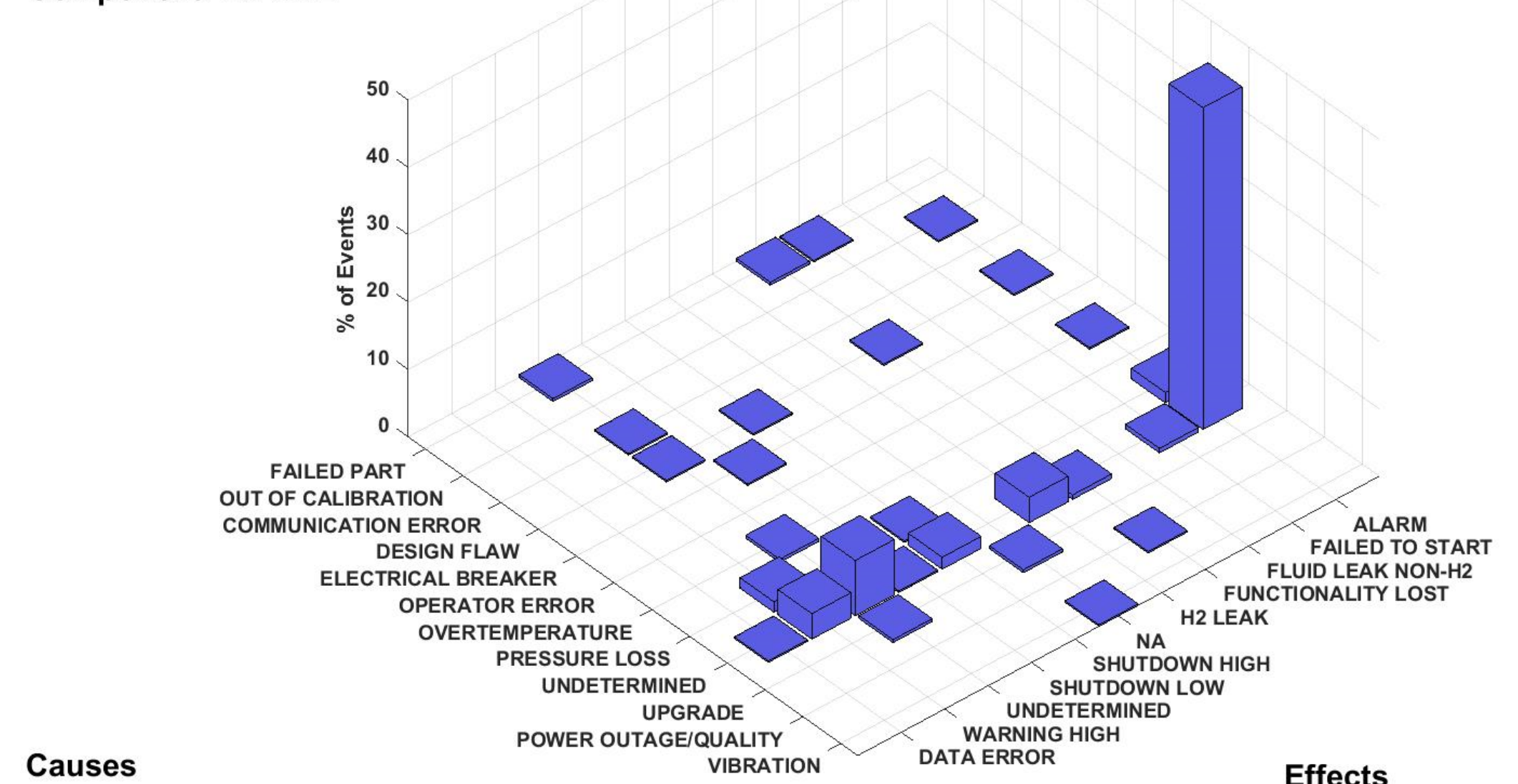

Causes

Created: May-05-18 7:44 AM | Data Range: 2014Q3-2017Q
NREL cdpRETAIL_inf_70

Effects

Preventative Maintenance accounted for $25 \%$ of all events. 


\section{Maintenance Causes and Effects: Compressor (Valve)} \\ Subsystem: COMPRESSOR \\ Component: VALVE}

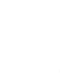

$1+N=$

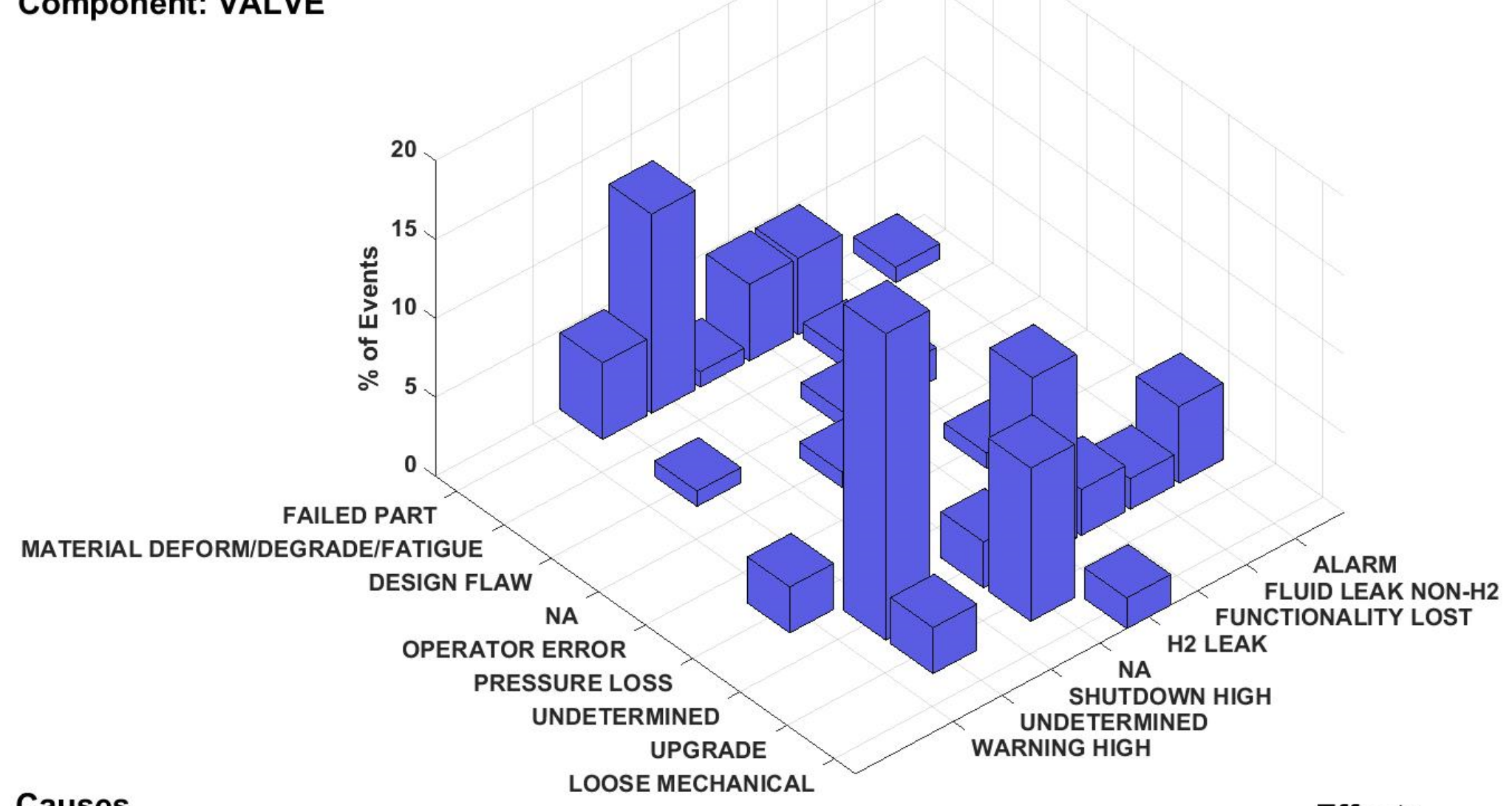

Causes

Effects 


\section{CDP-INFR-72}

\section{Maintenance Causes and Effects: Compressor (Fitting)}

Maintenance Causes and Effects - Retail Stations

Subsystem: COMPRESSOR

Component: FITTING

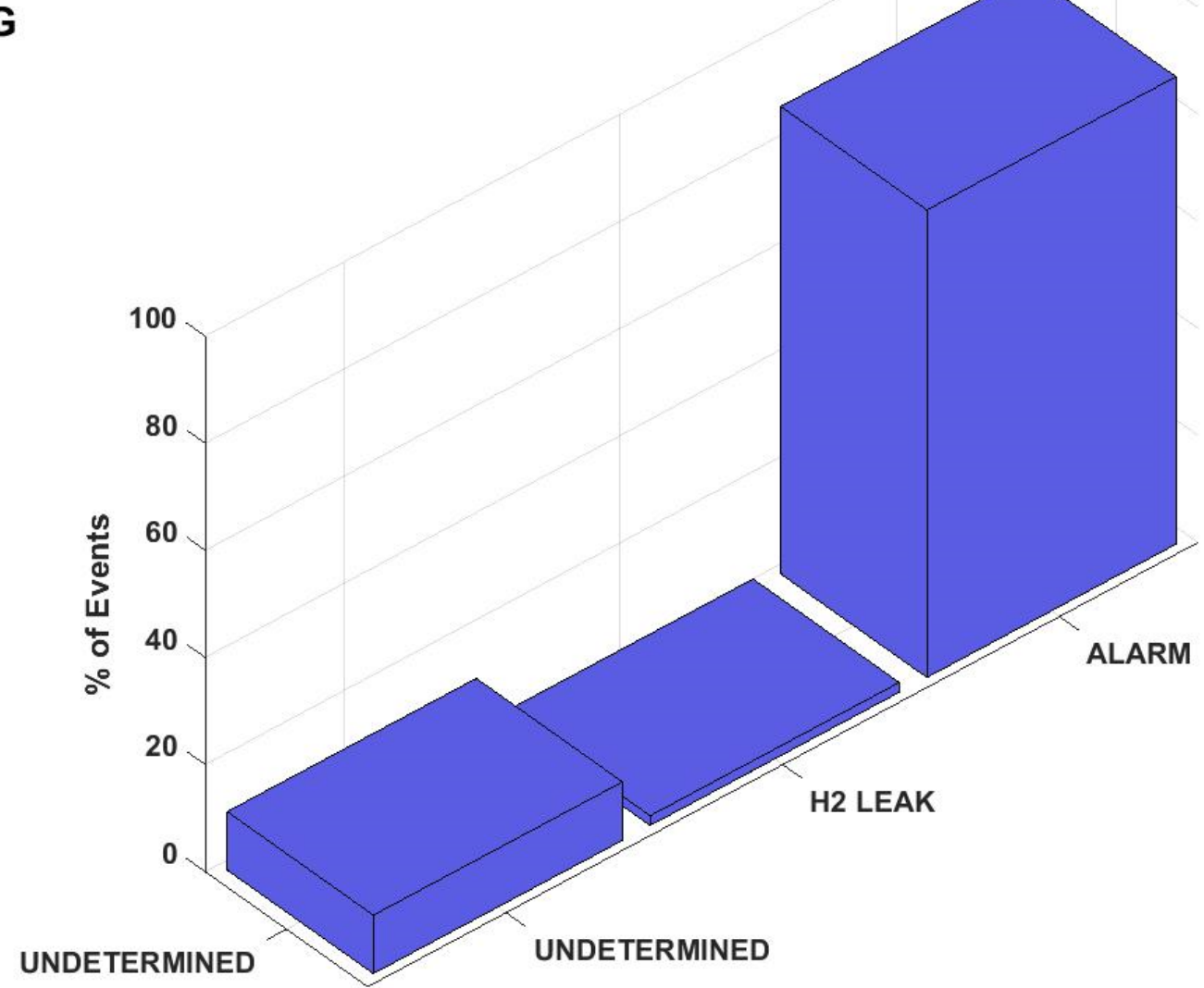

Causes

Effects

NREL cdpRETAIL_infr_72

Created: May-05-18 7:31 AM | Data Range: 2014Q3-2017Q4 
Performance 


\section{CDP-INFR-01 Hydrogen Dispensed by Quarter}

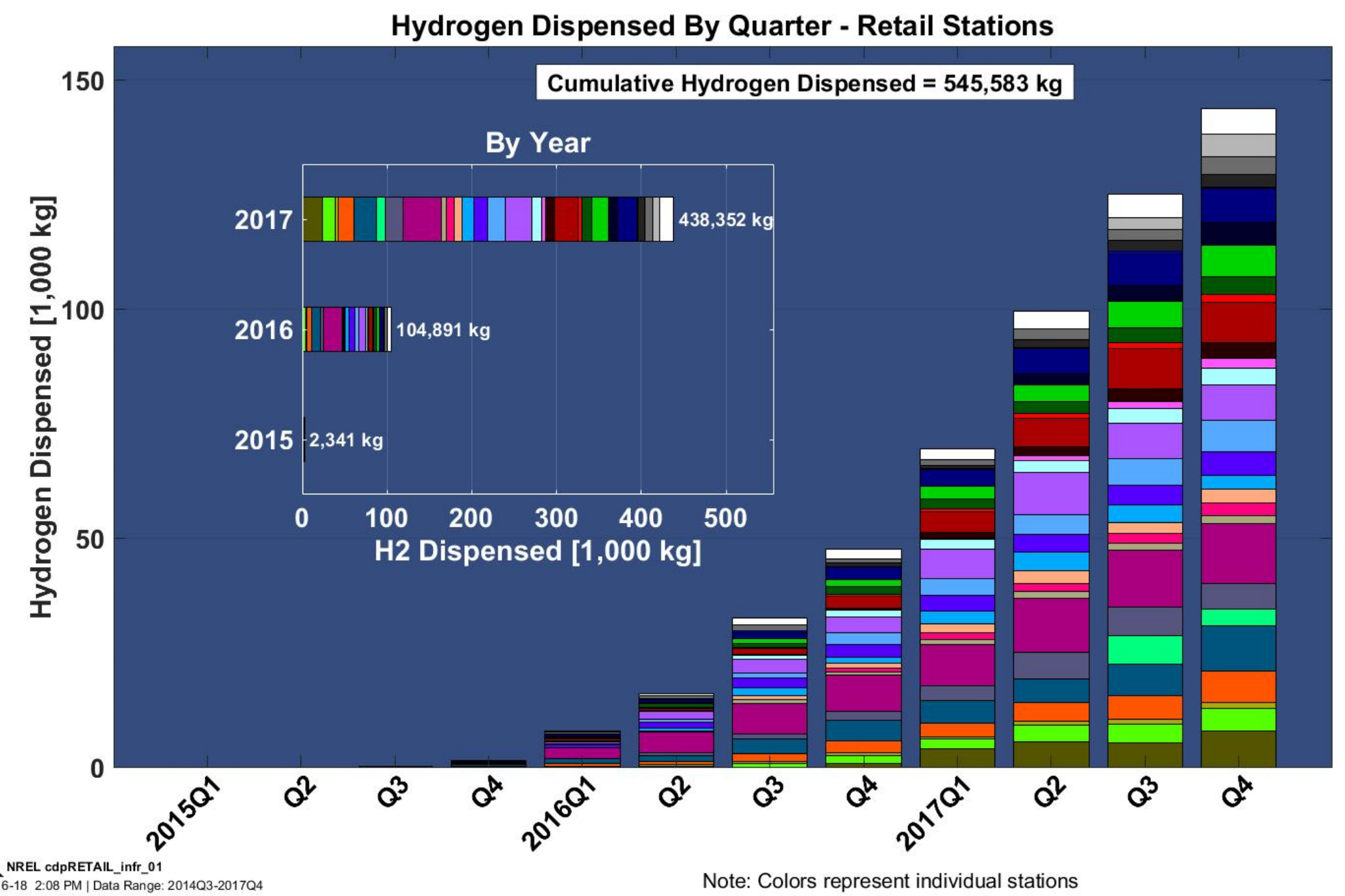




\section{CDP-INFR-58 \\ Hydrogen Fills by Quarter}

Hydrogen Fills By Quarter - Retail Stations

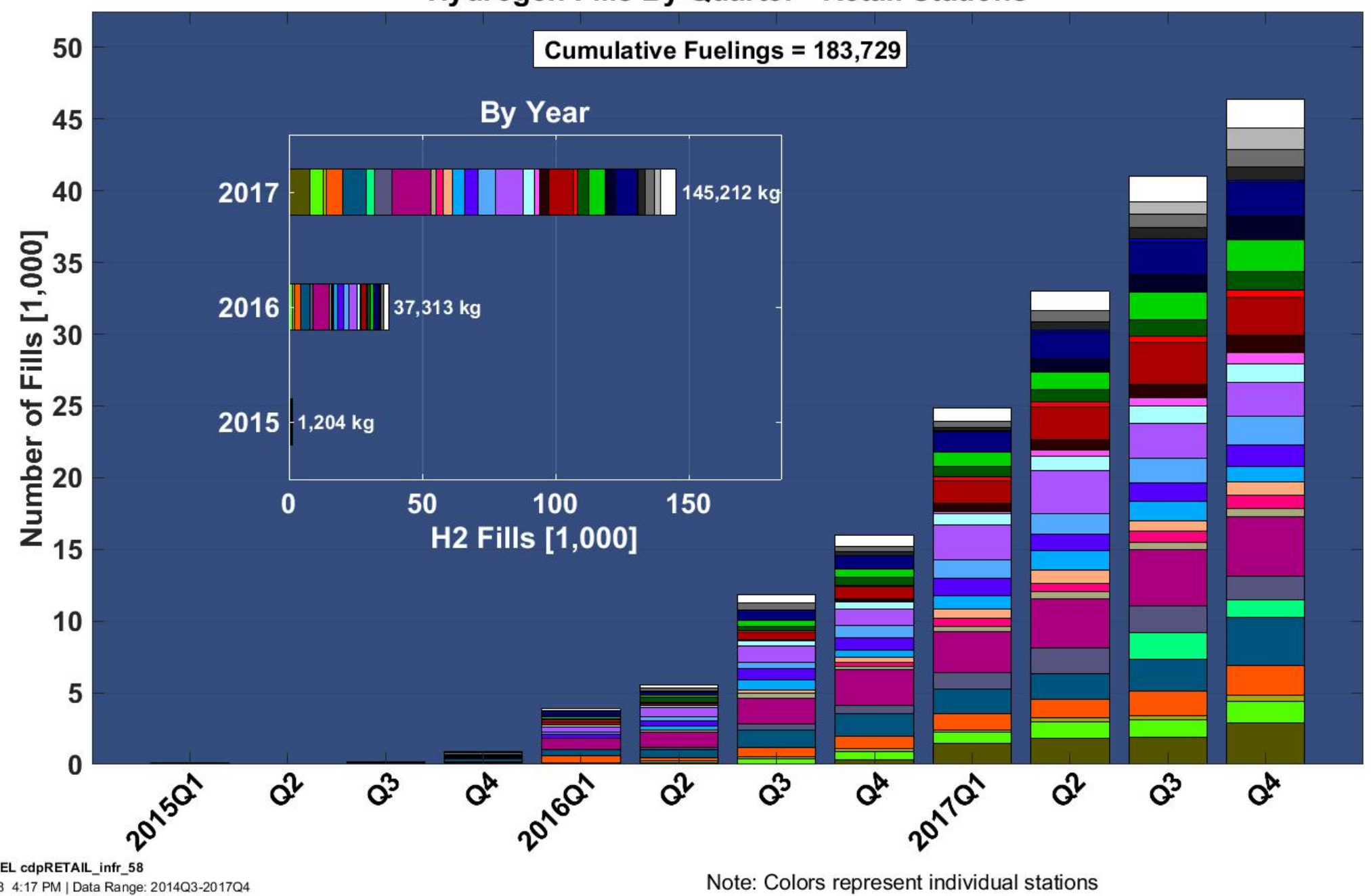




\section{CDP-INFR-81 Hydrogen Dispensed by Region}

Hydrogen Dispensed By Region - Retail Stations

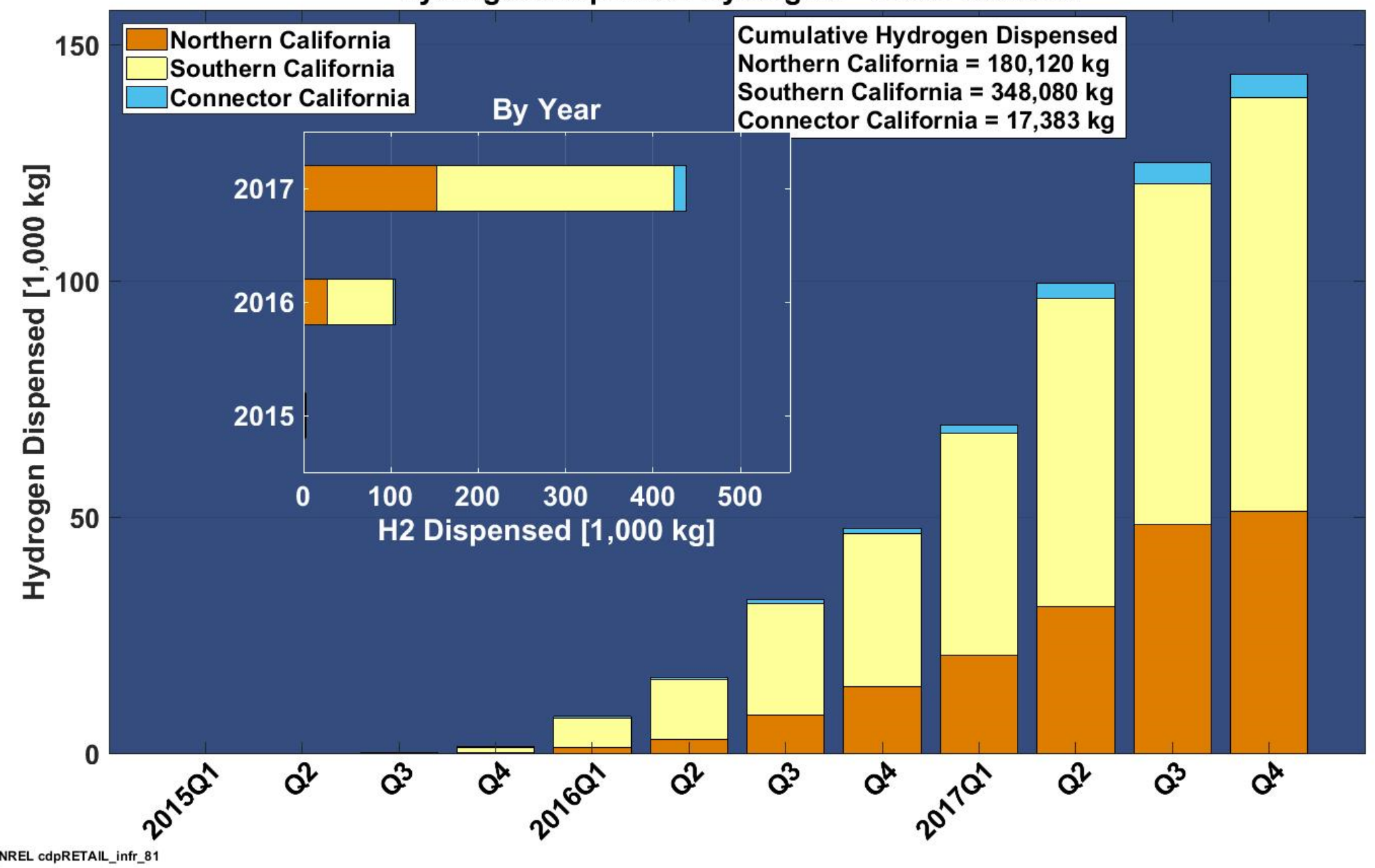




\section{CDP-INFR-02 Histogram of Fueling Rates}

Histogram of Fueling Rates

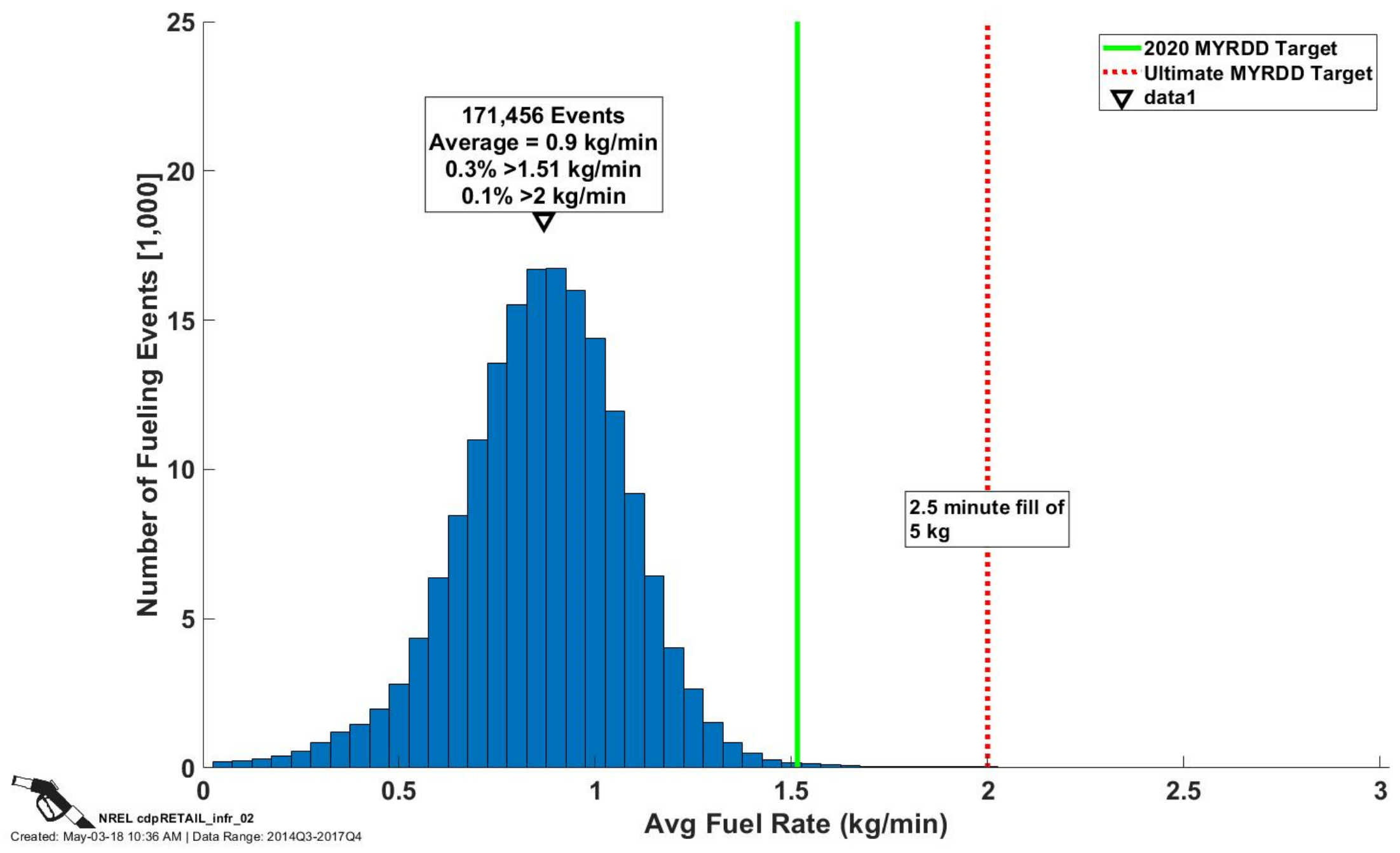




\section{CDP-INFR-03 Histogram of Fueling Times}

\section{Histogram of Fueling Times}

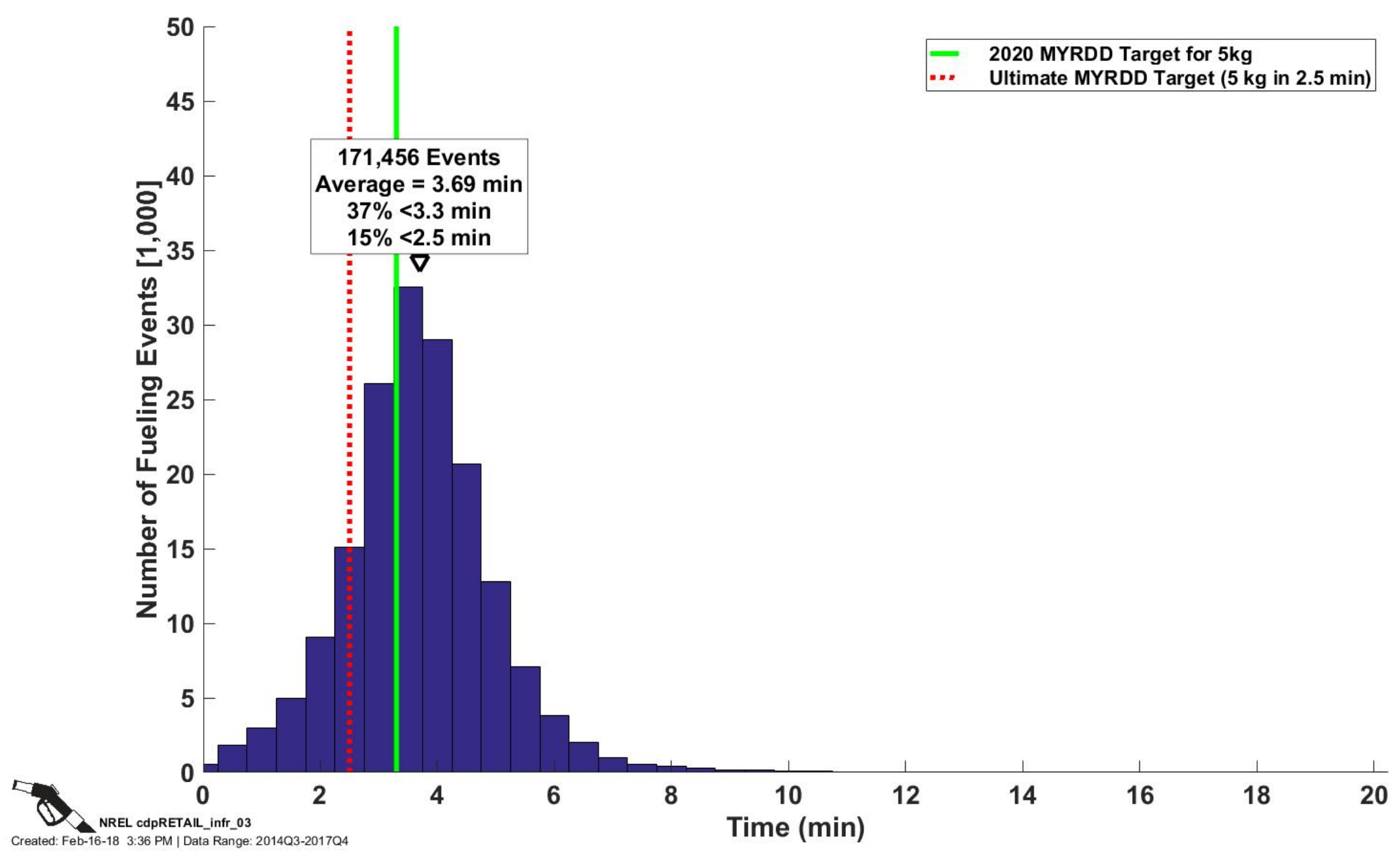




\section{CDP-INFR-04 \\ Histogram of Fueling Amounts}

\section{Histogram of Fueling Amounts}

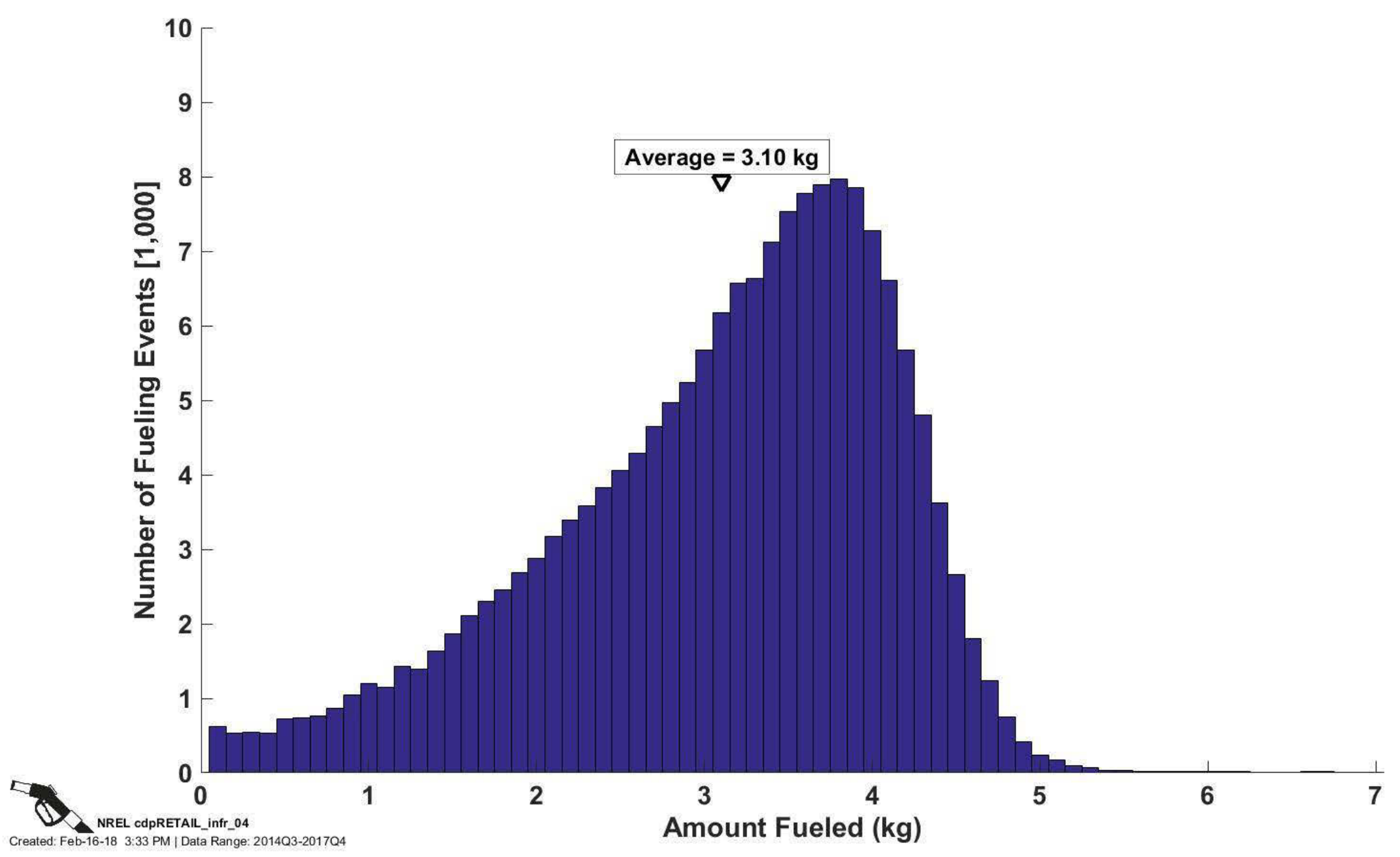




\section{CDP-INFR-08 \\ Time Between Fueling}
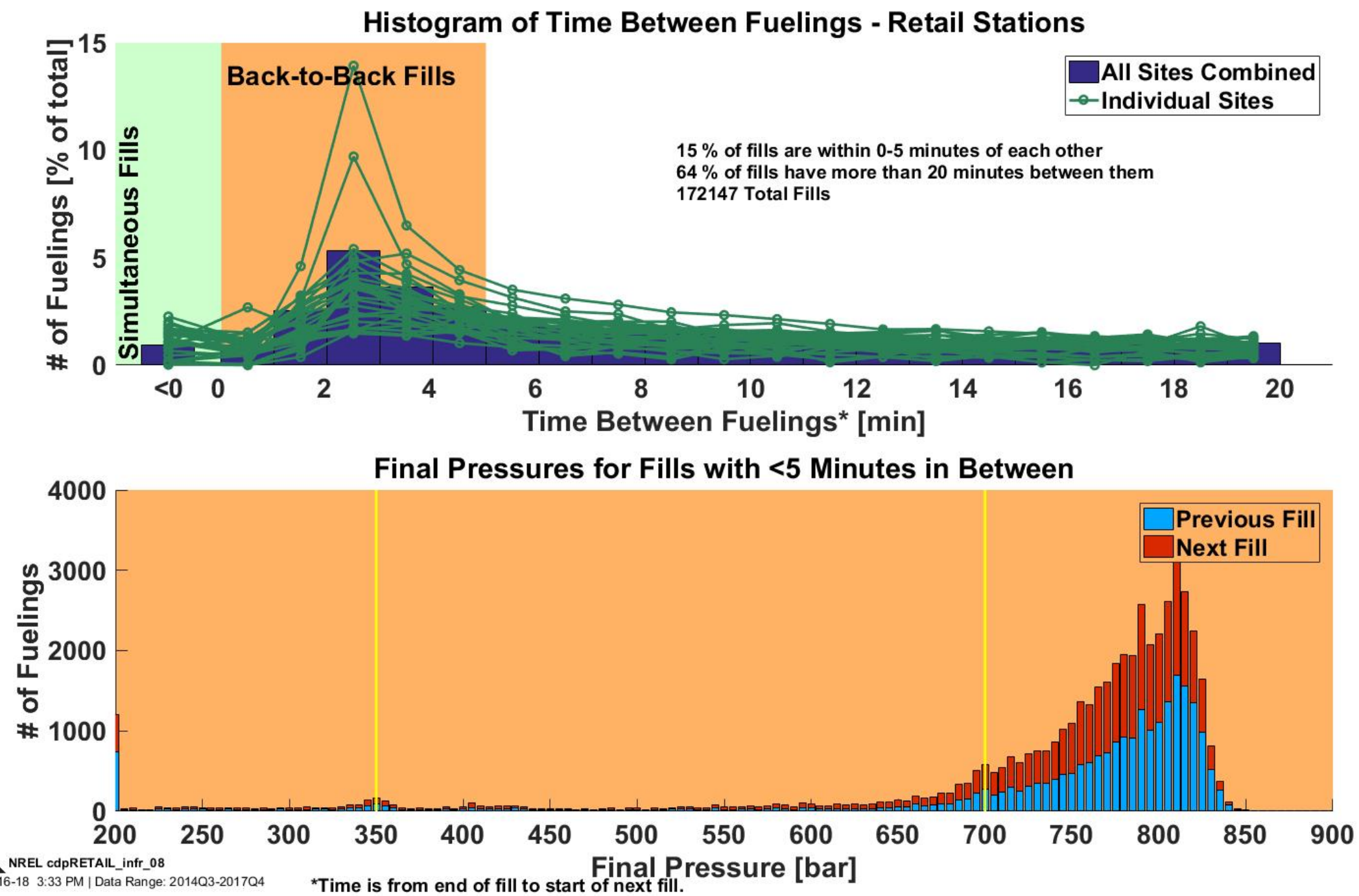


\section{CDP-INFR-09 \\ Fueling Final Pressures}

Fueling Final Pressures - Retail Stations

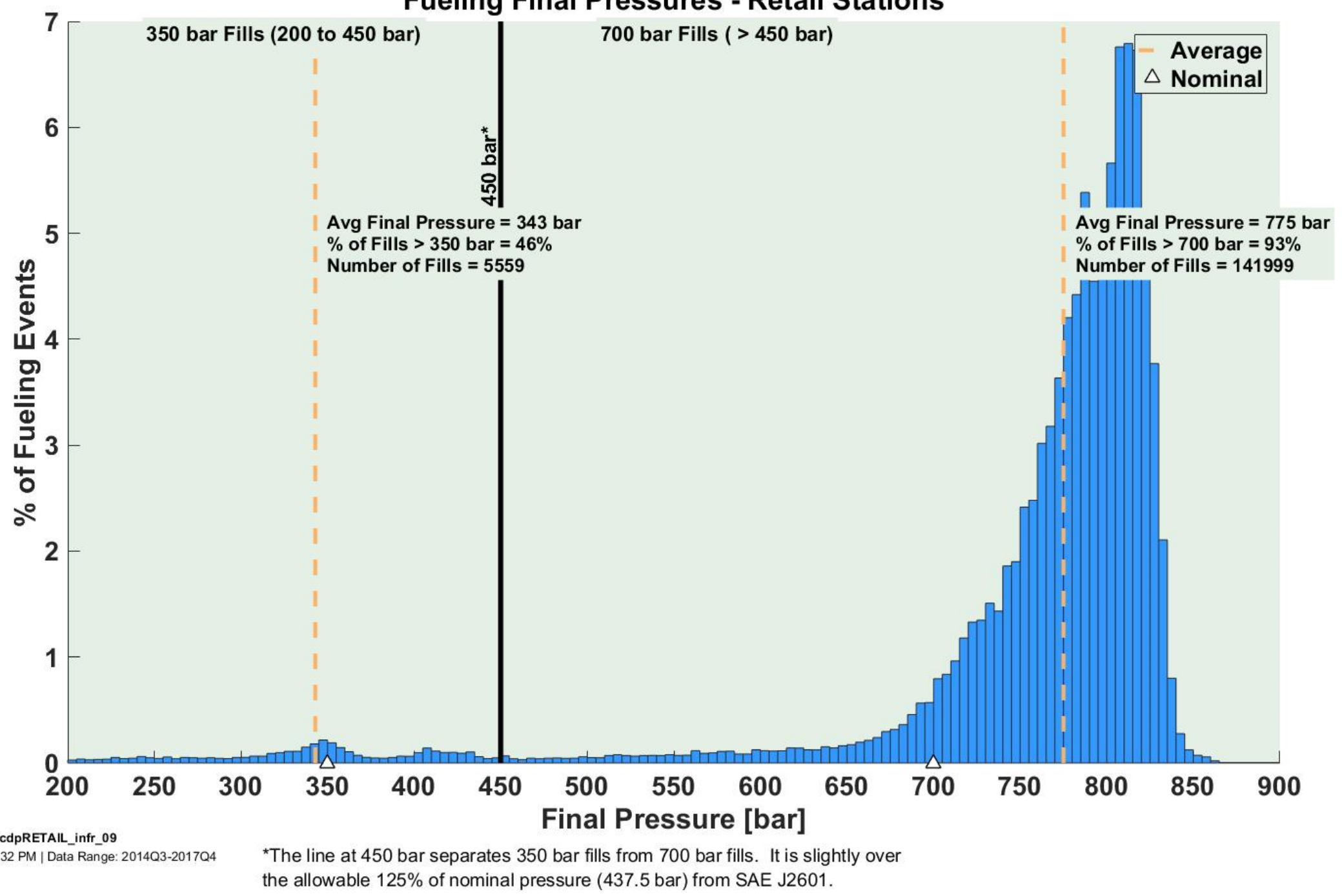




\section{CDP-INFR-12 \\ Fueling Rates 350 bar vs. 700 bar}

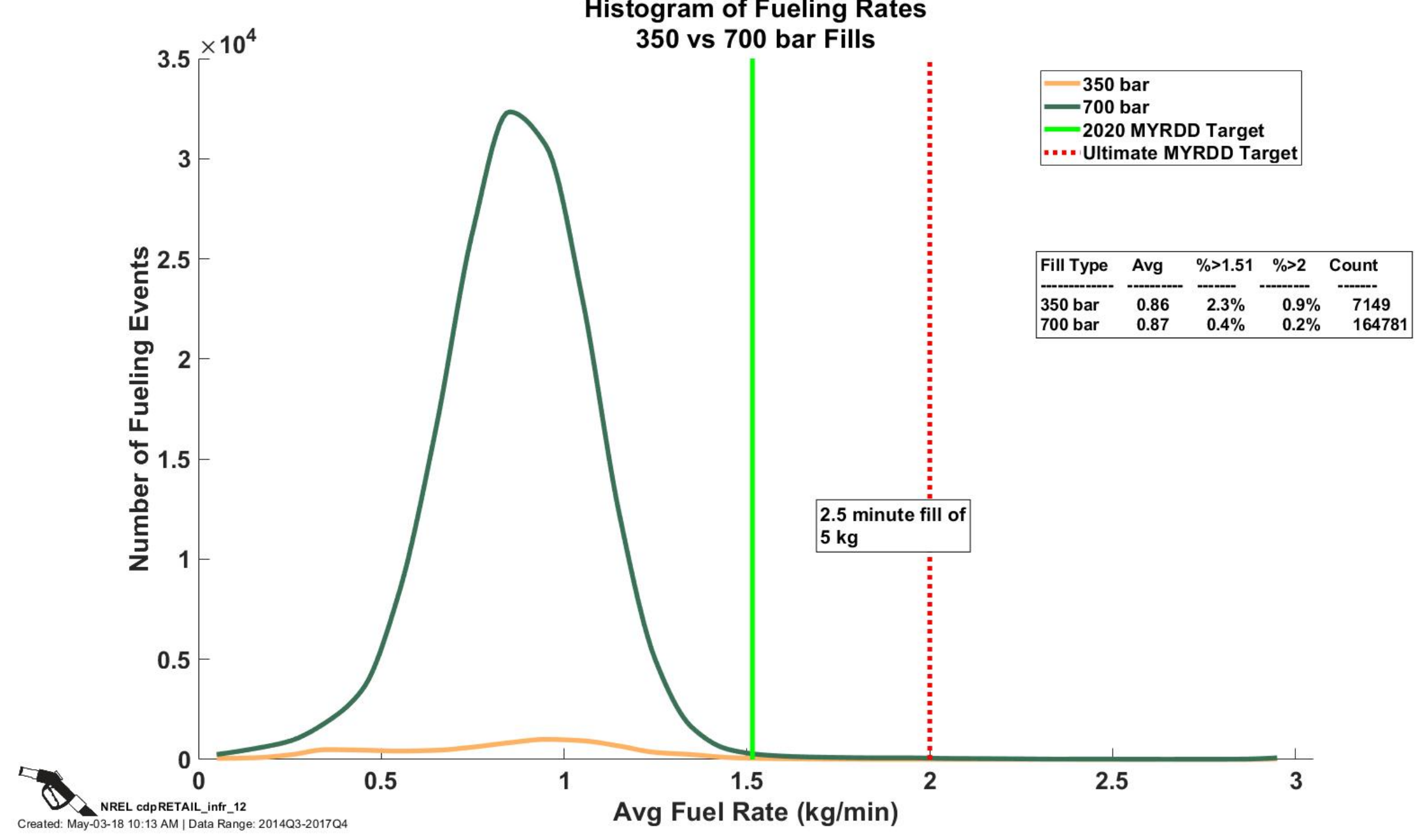




\section{Hydrogen Dispensed by Quarter and Pressure}

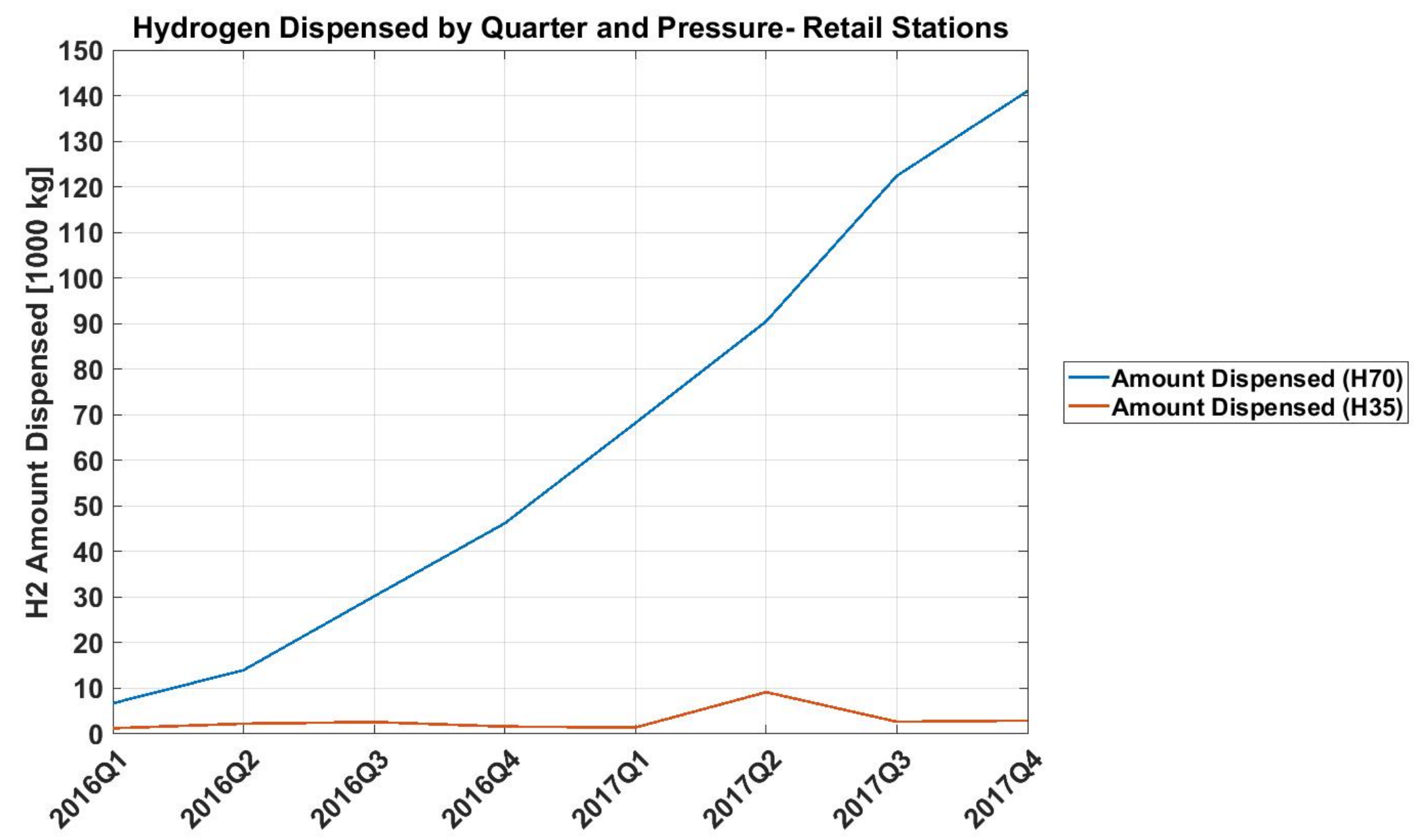




\section{Number of Fueling Events per Hour}

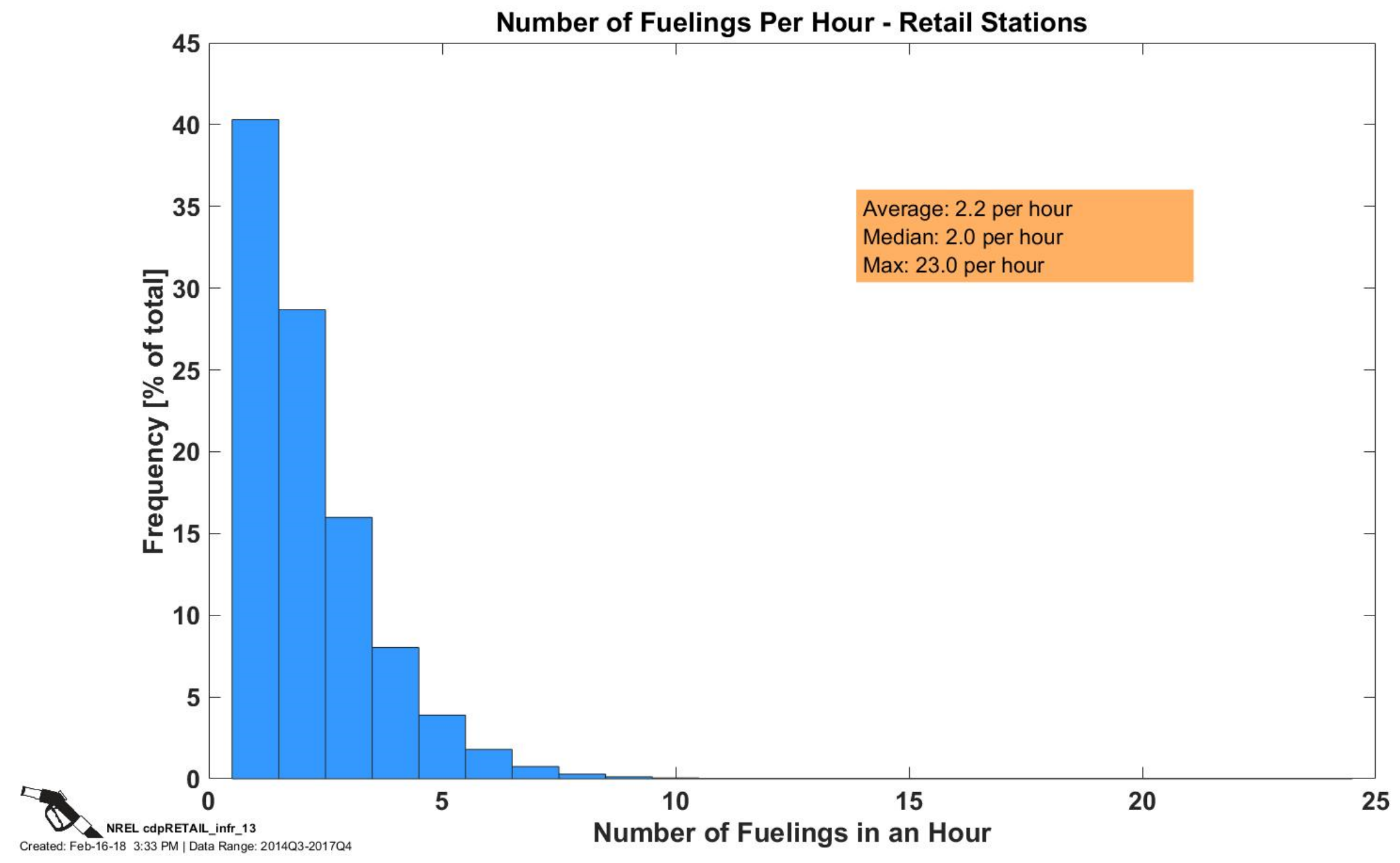




\section{Hydrogen Dispensed per Hour}

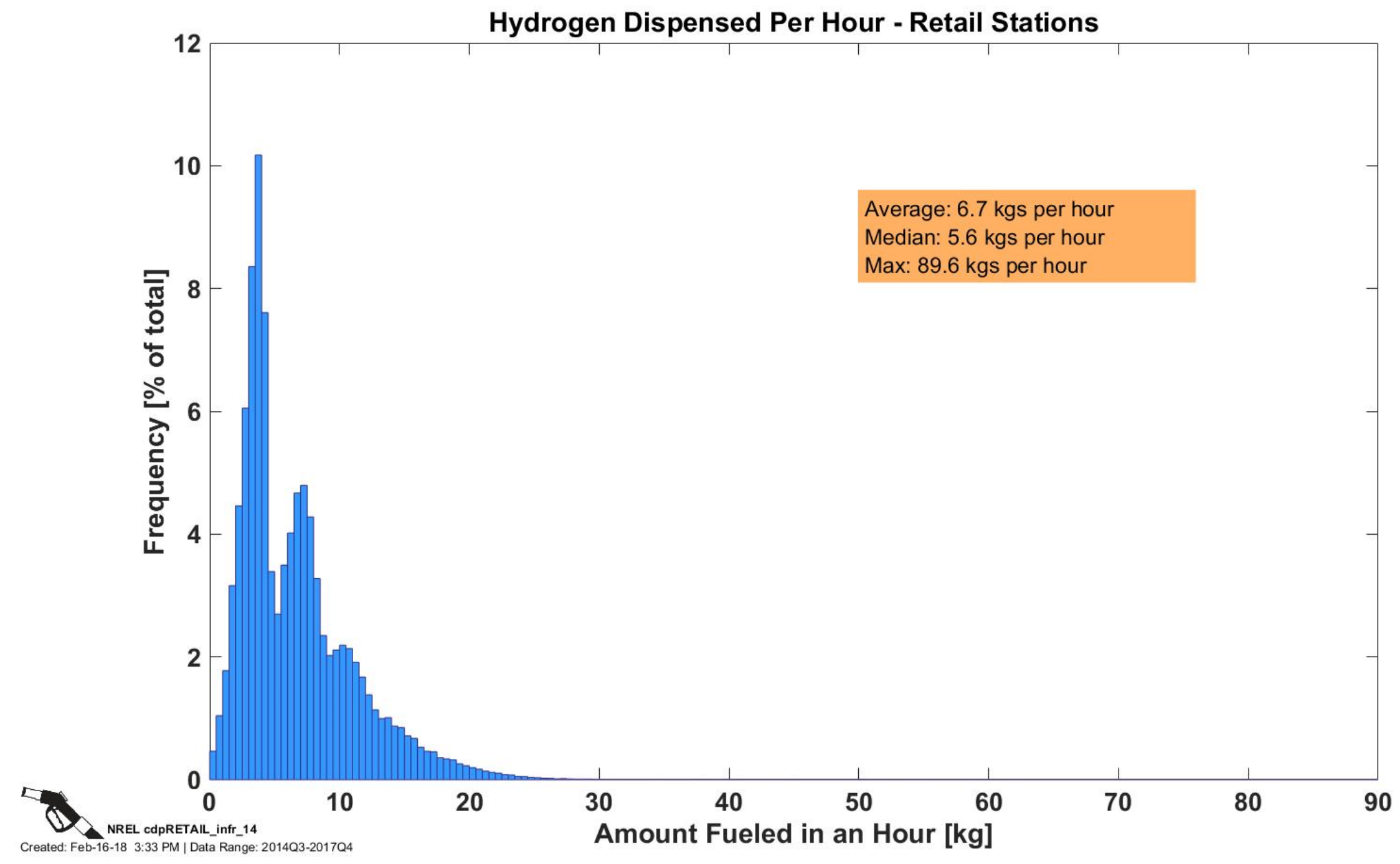




\section{Number of Fills by Time of Day}

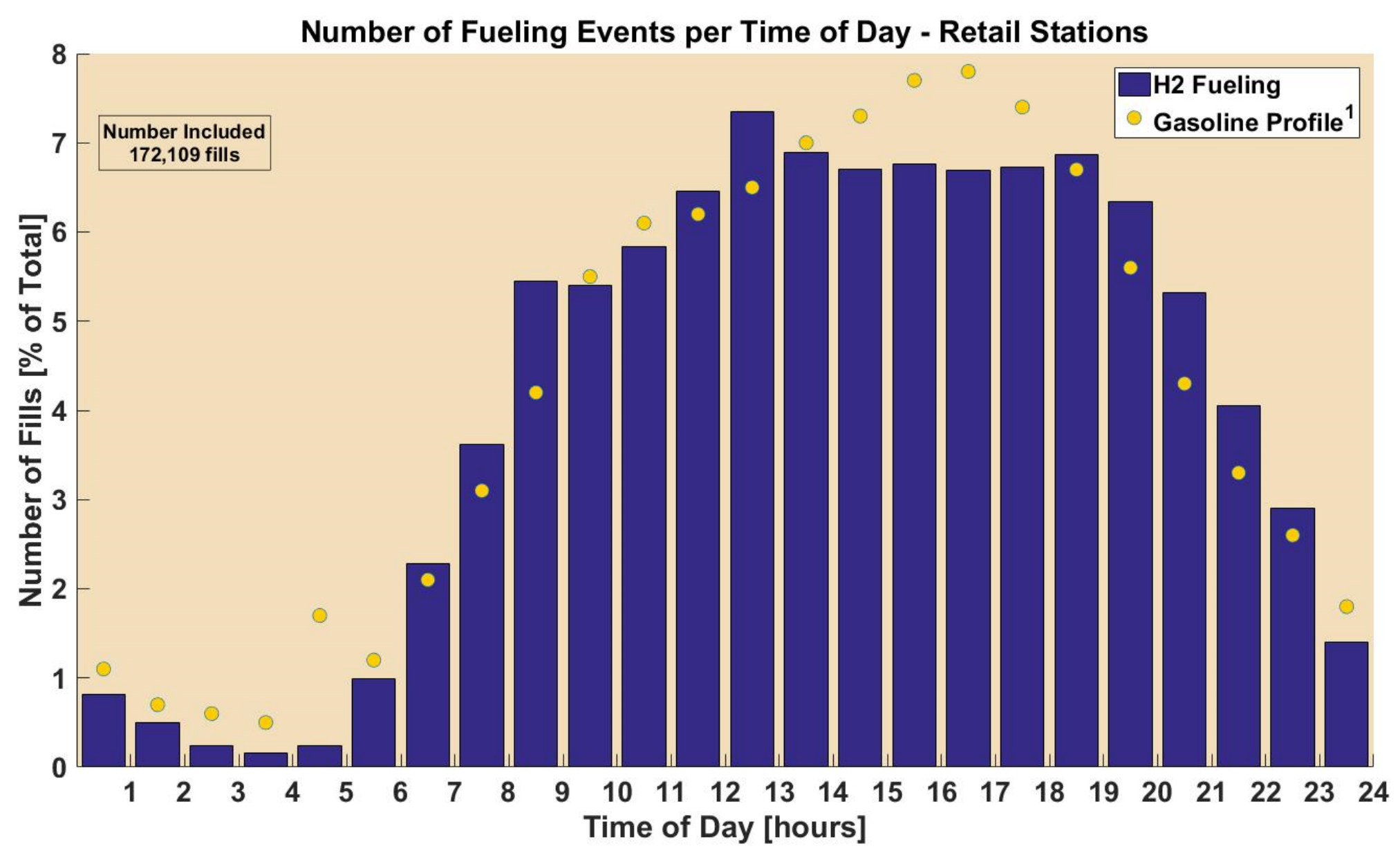




\section{CDP-INFR-16}

\section{Fueling Amounts per Time of Day}

Fueling Amounts per Time of Day - Retail Stations

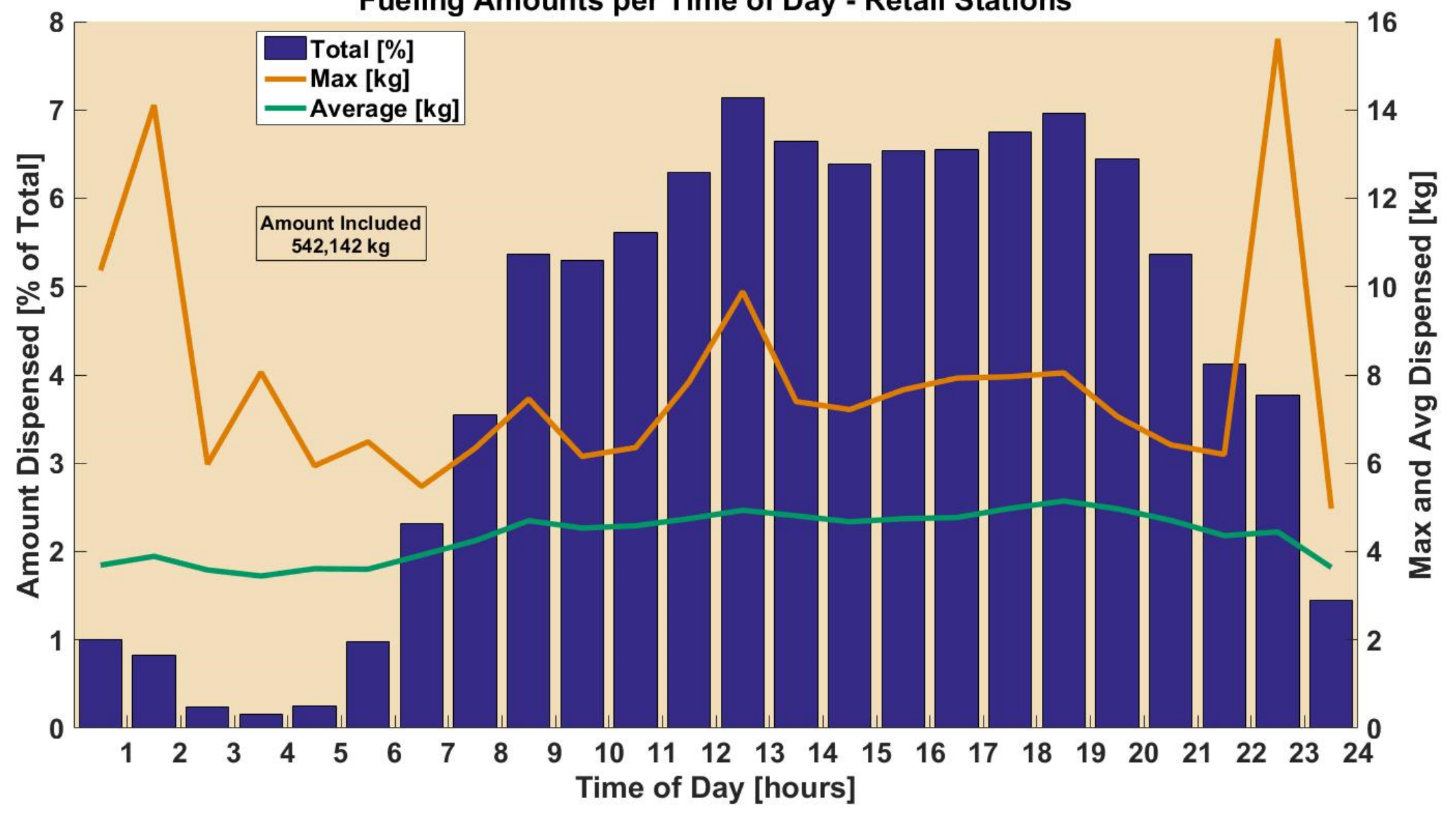

NREL cdpRETAIL_infr_16 


\section{CDP-INFR-88a Fueling Profile by Day and Hour Connector/Destination Stations}

Fueling Amounts by Day and Hour - Retail Stations - Connector/Destination California
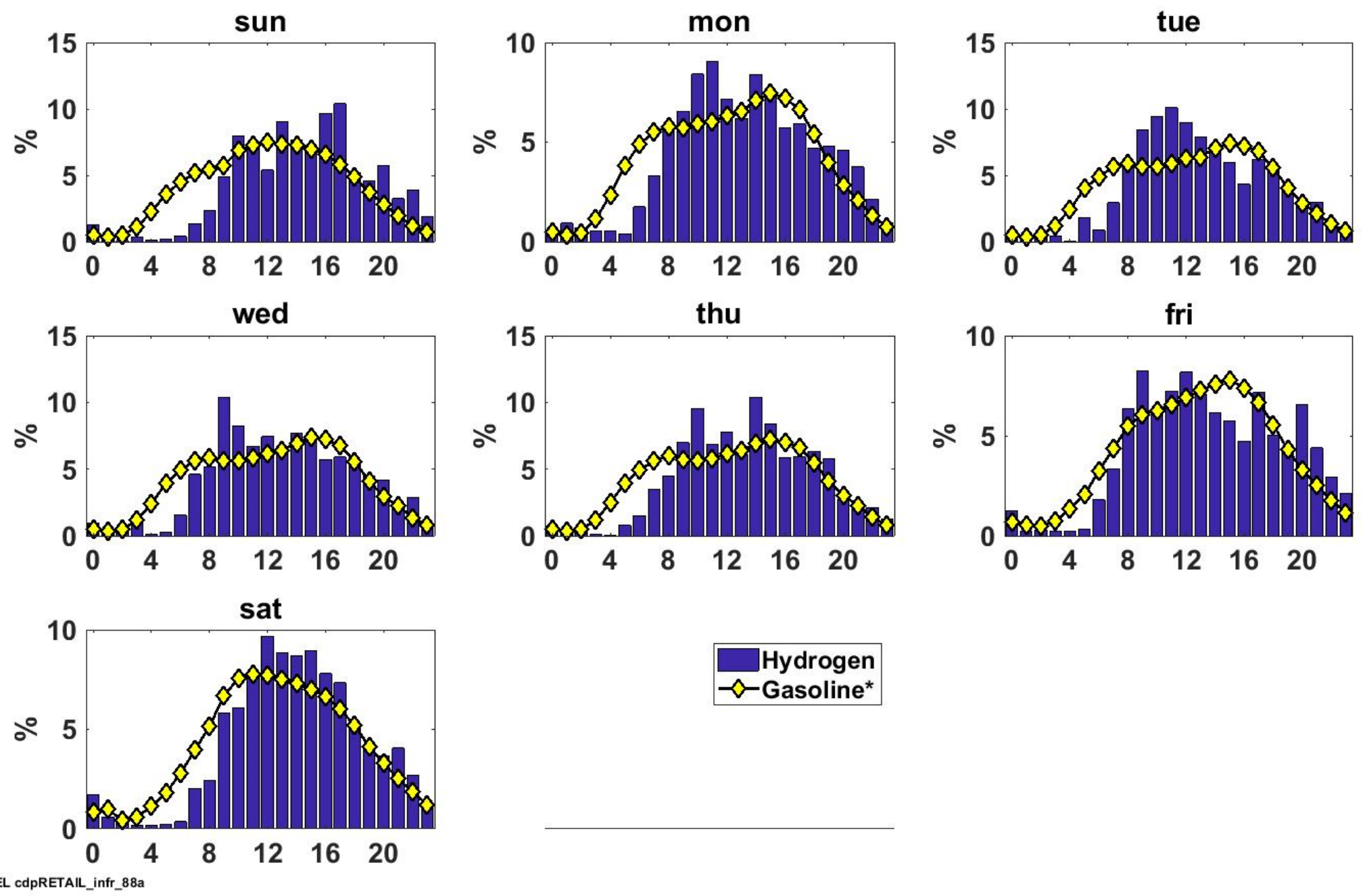


\section{CDP-INFR-88b Fueling Profile by Day and Hour Northern California}

Fueling Amounts by Day and Hour - Retail Stations - Northern California
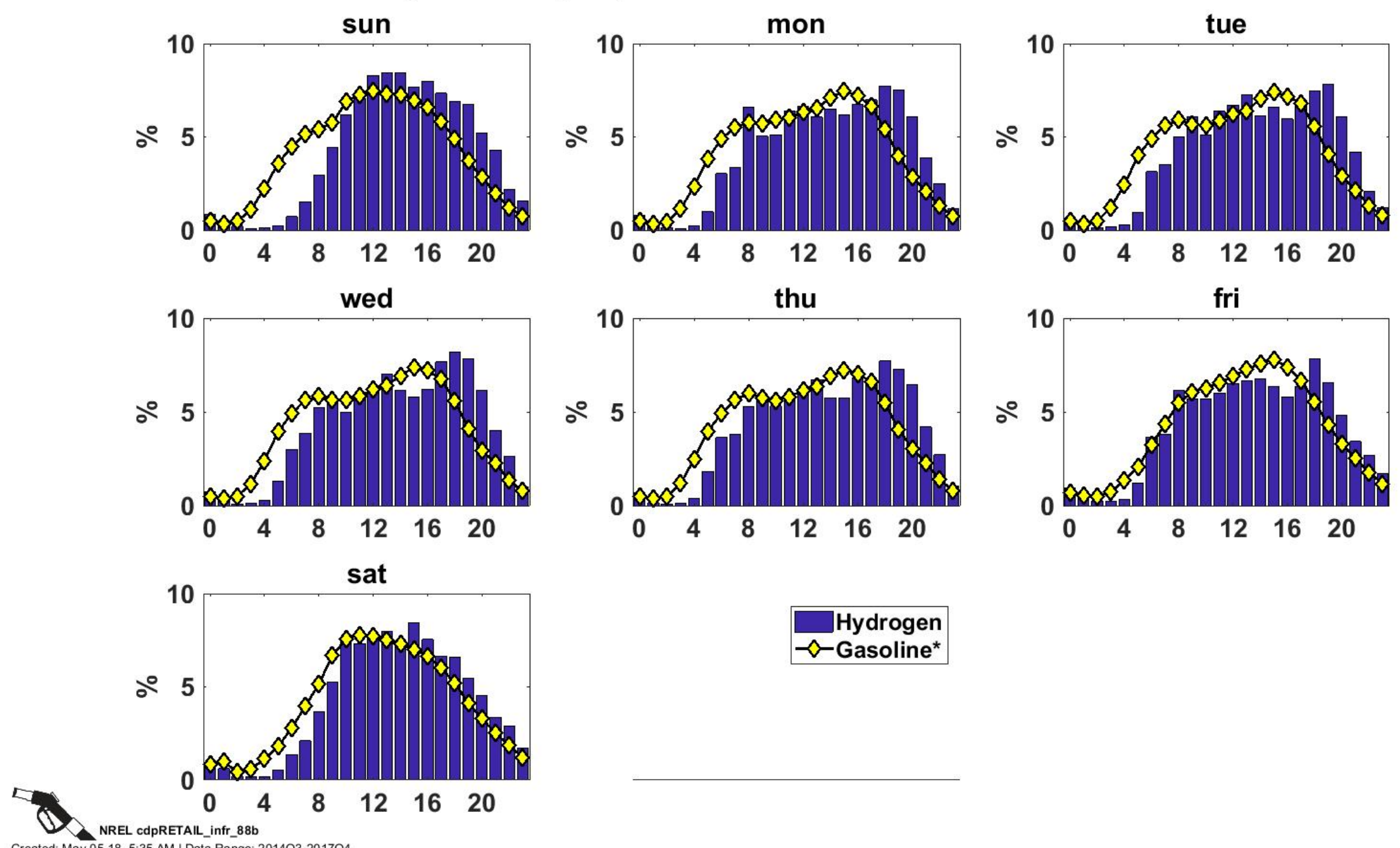


\section{CDP-INFR-88c Fueling Profile by Day and Hour Southern California}

Fueling Amounts by Day and Hour - Retail Stations - Southern California
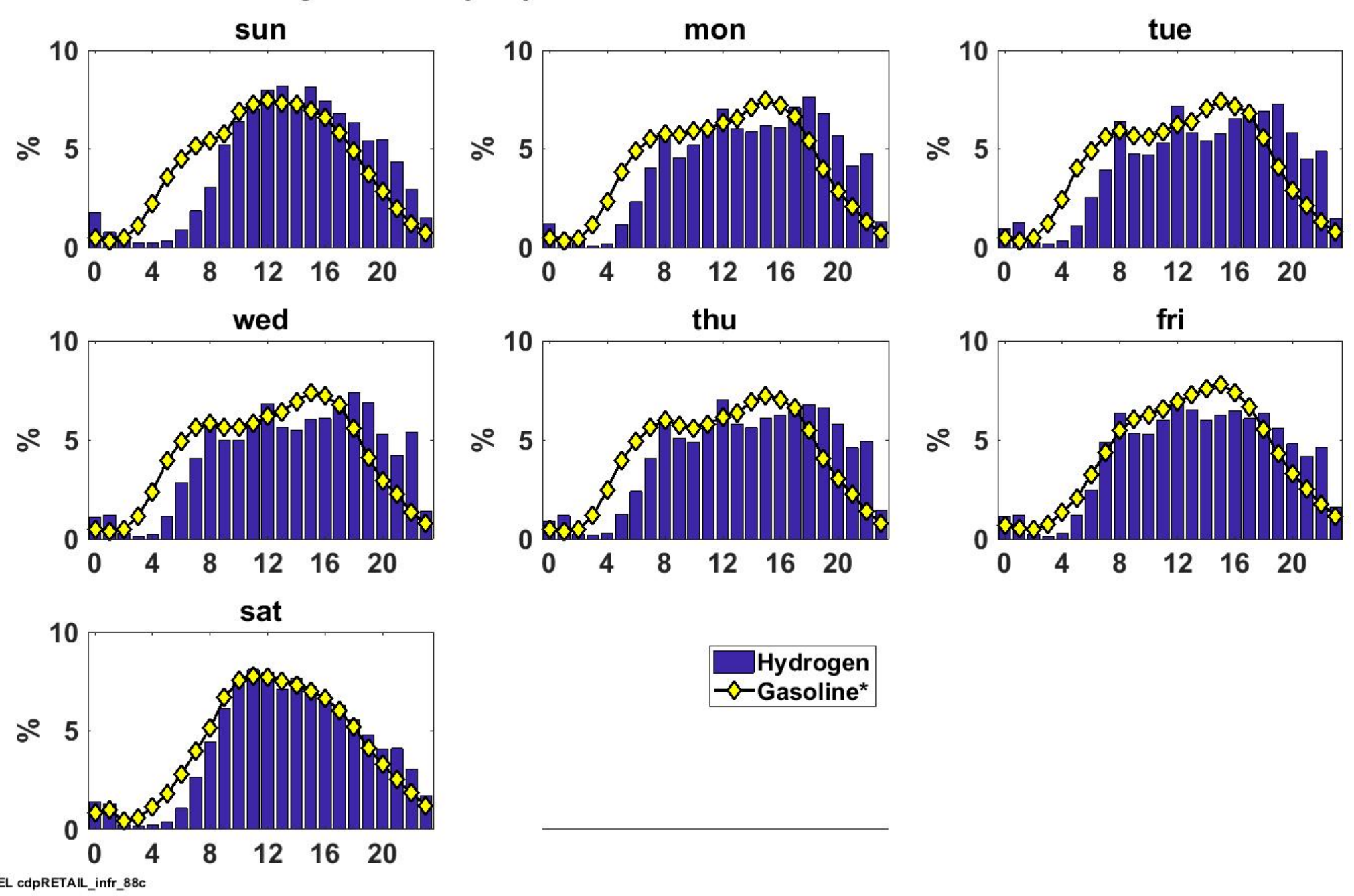


\section{CDP-INFR-91 \\ Missed Fuel Opportunity}

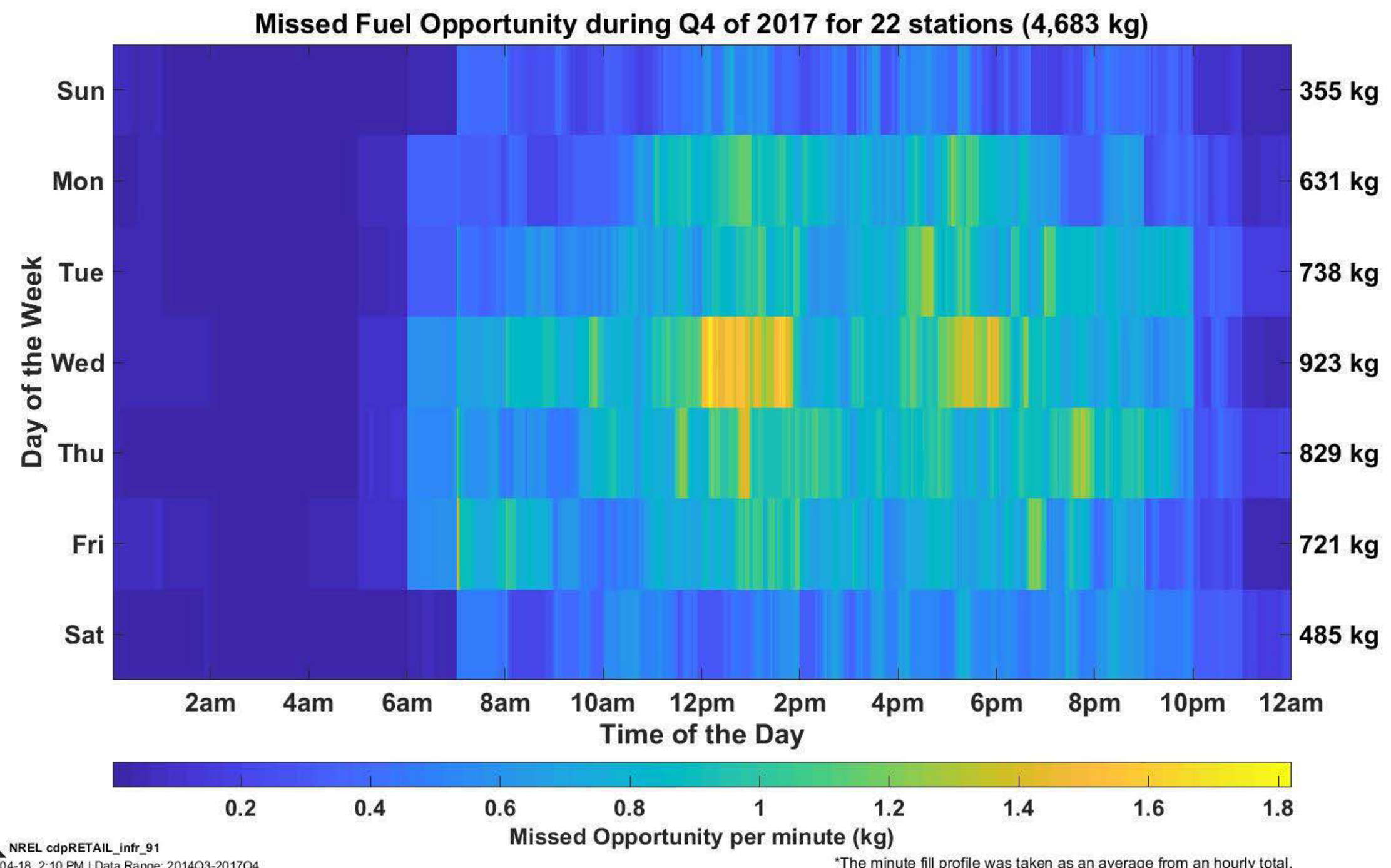

*The minute fill profile was taken as an average from an hourly total. 


\section{CDP-INFR-17}

\section{Fueling Rates by Amount Filled}

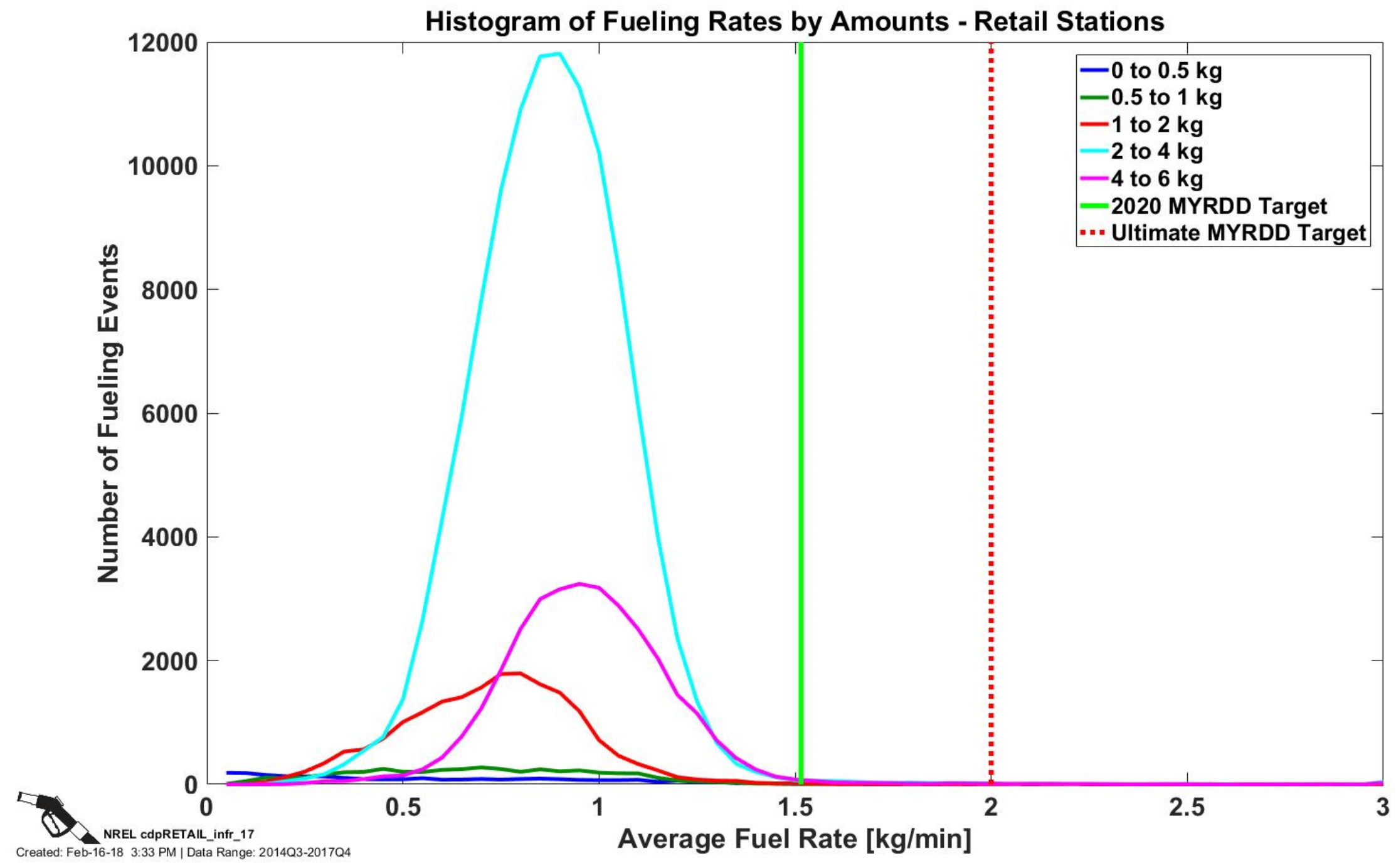




\section{CDP-INFR-18}

\section{Fueling Amount vs. Time to Fill}
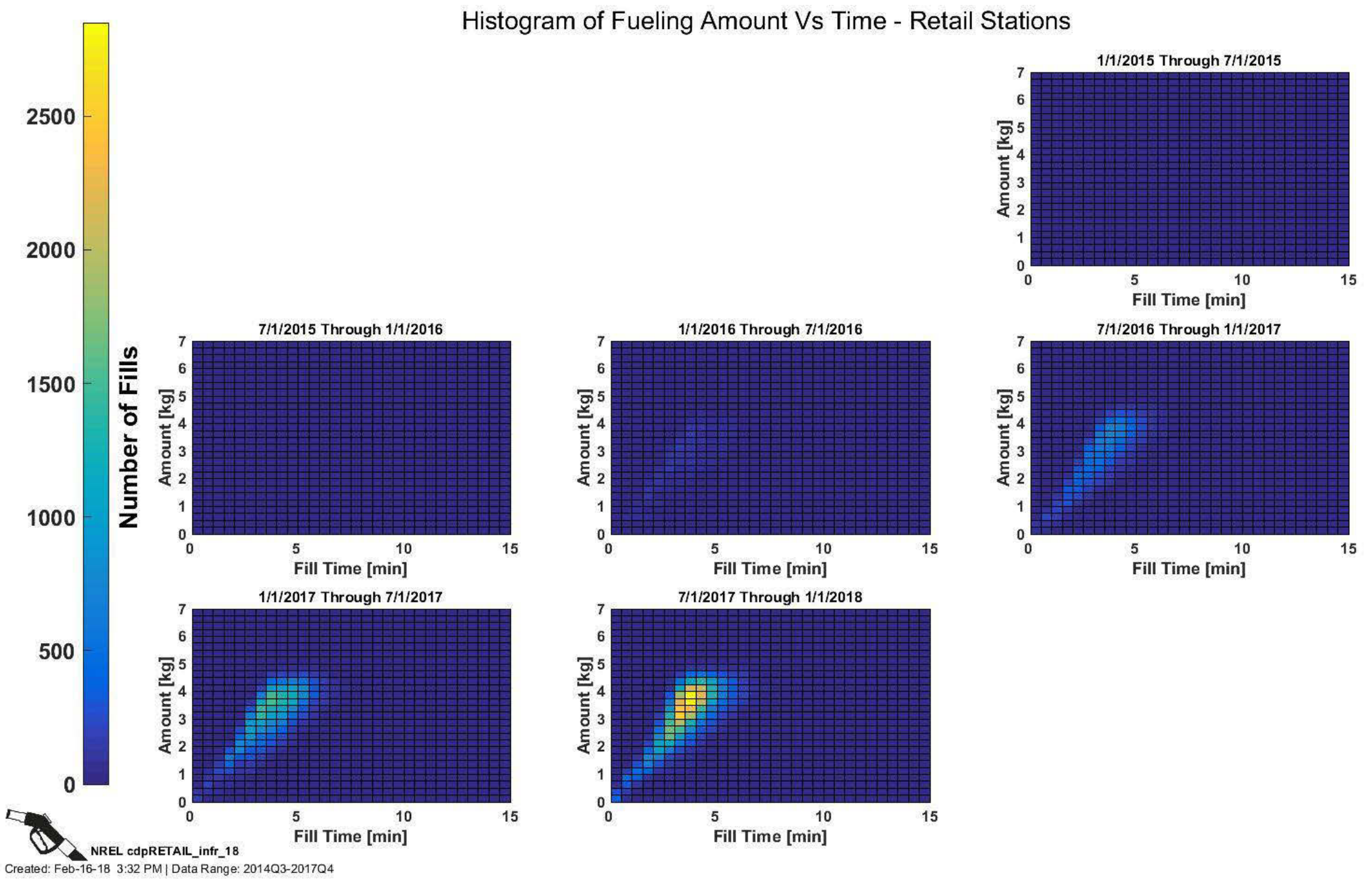


\section{CDP-INFR-56 \\ Fueling Rates by Year}

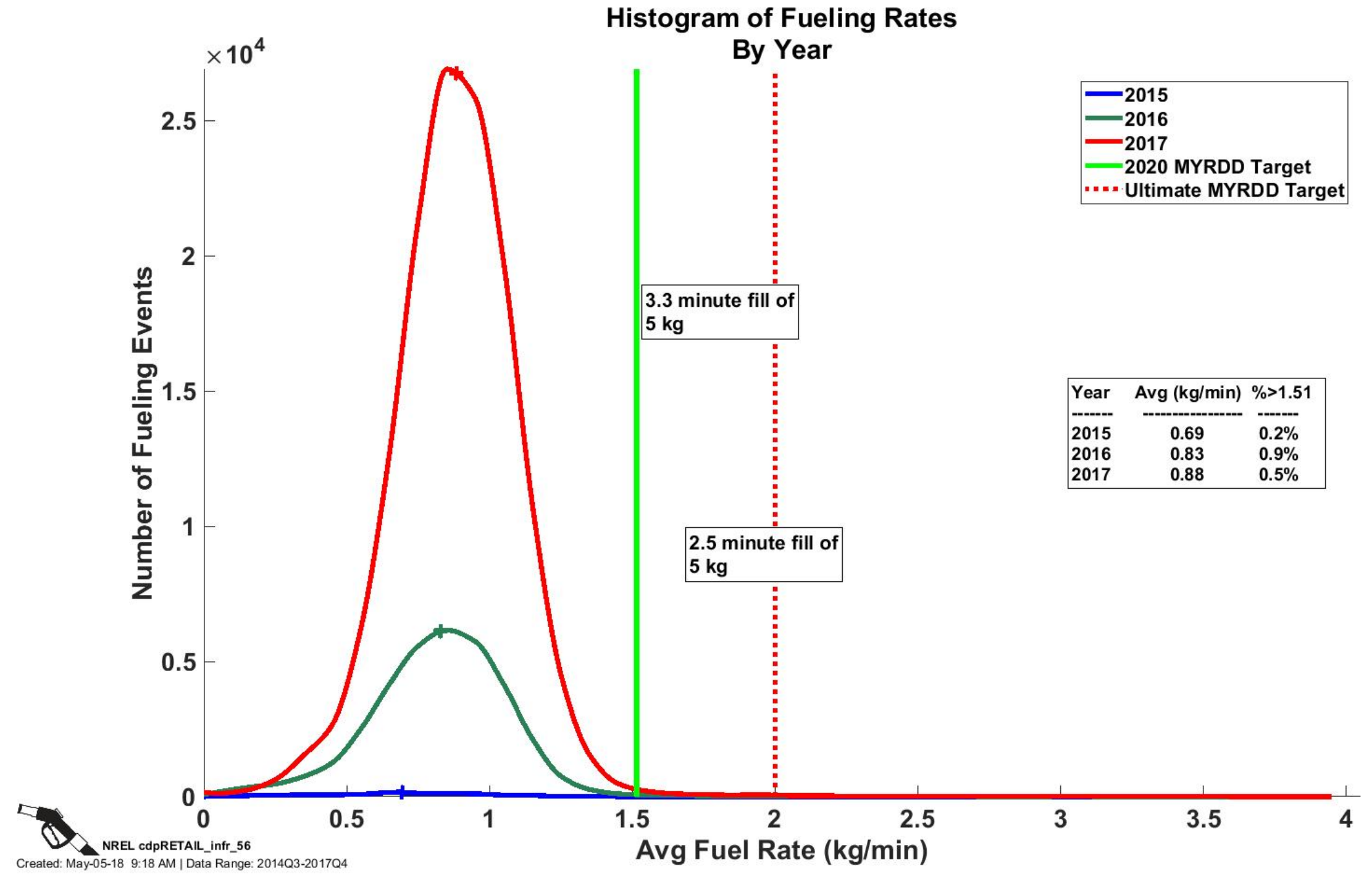




\section{CDP-INFR-55 \\ Monthly Averages: All Fills}
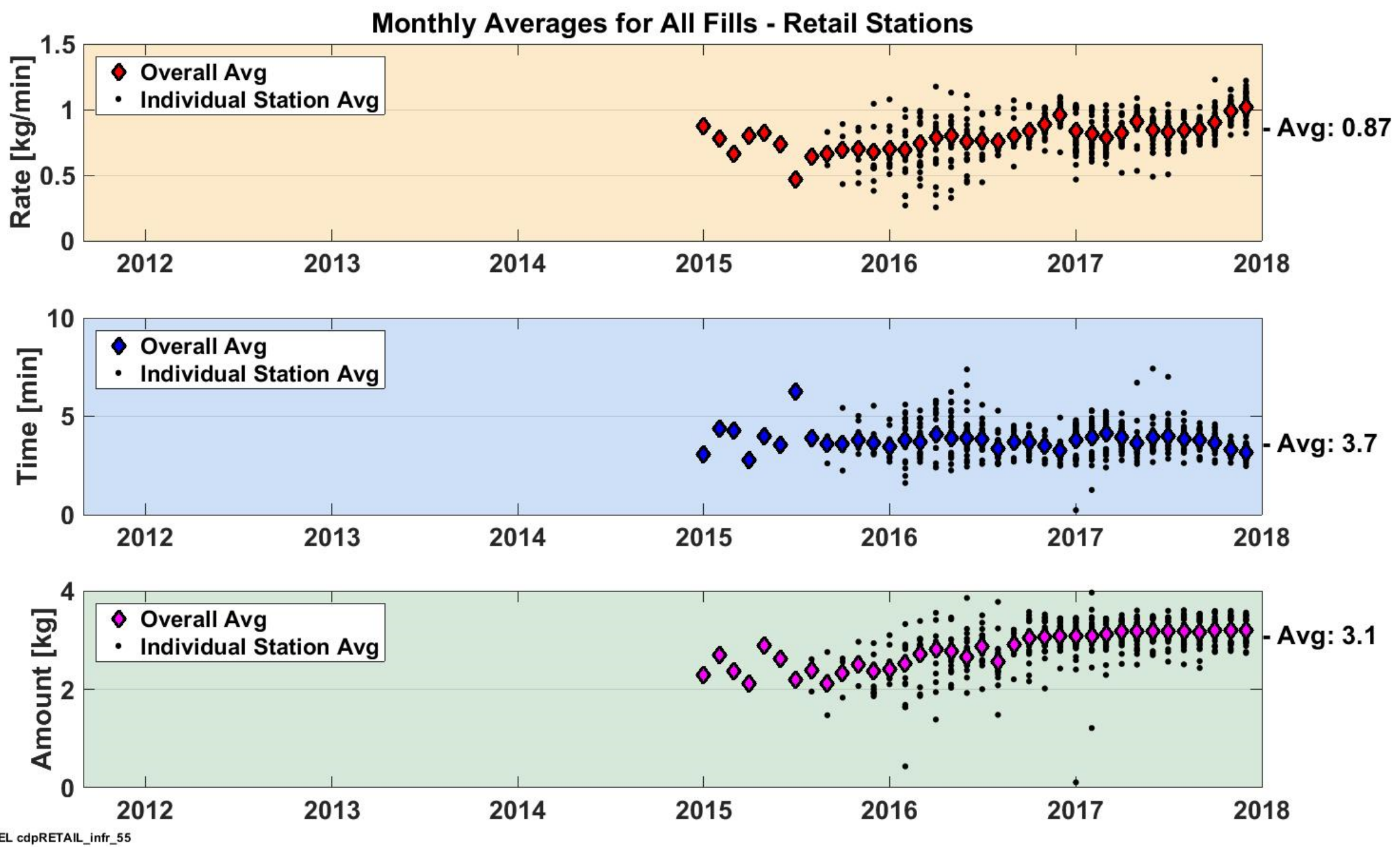


\section{CDP-INFR-57}

\section{Monthly Averages: 700 bar Fills $>1 \mathrm{~kg}$ with Pre-Cool of $-40^{\circ} \mathrm{C}$}
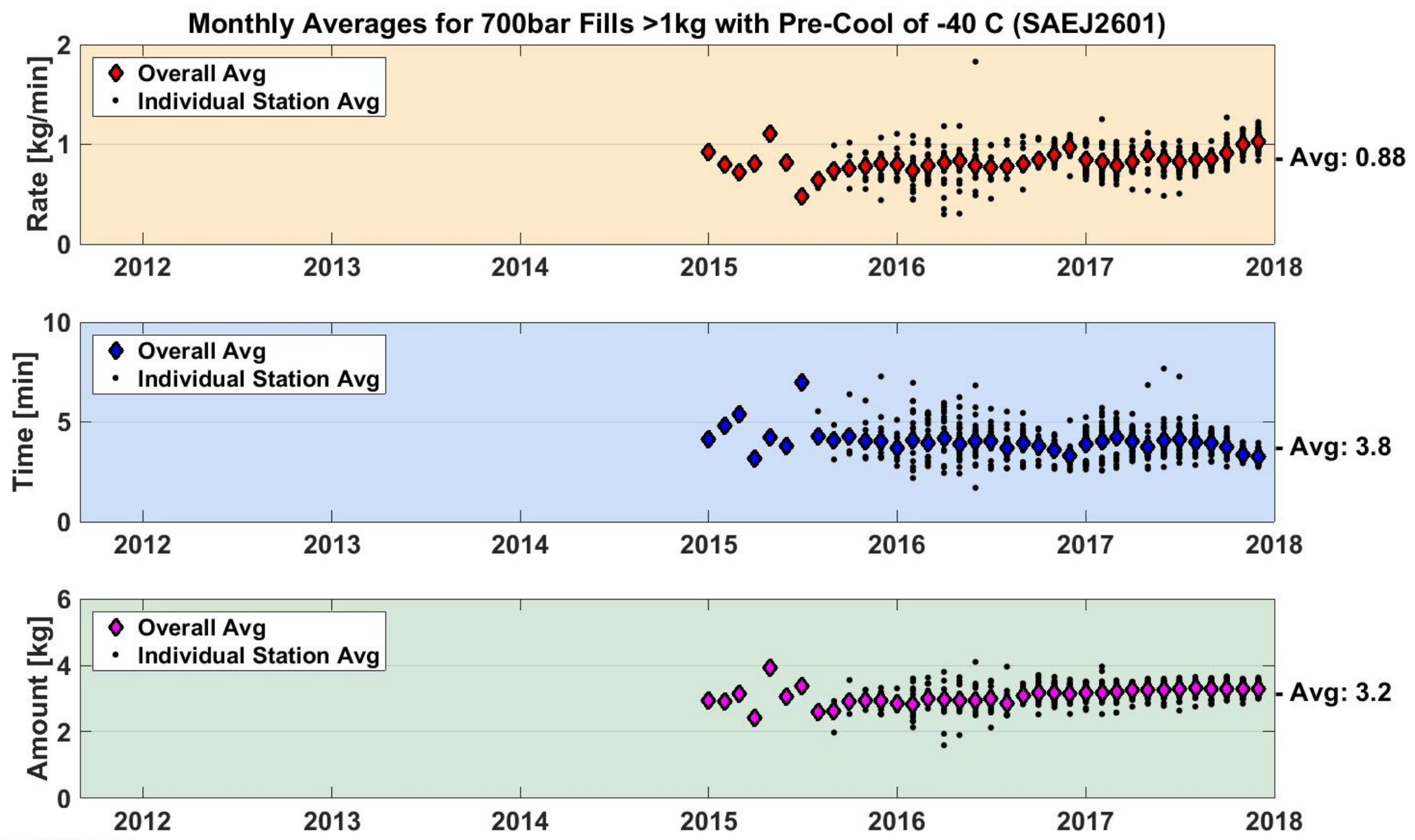


\section{Cost}

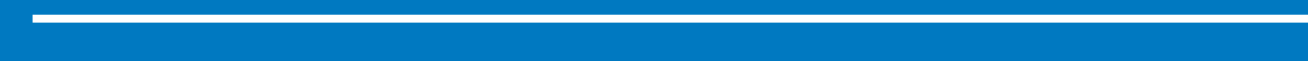




\section{Compressor Operation Cost}

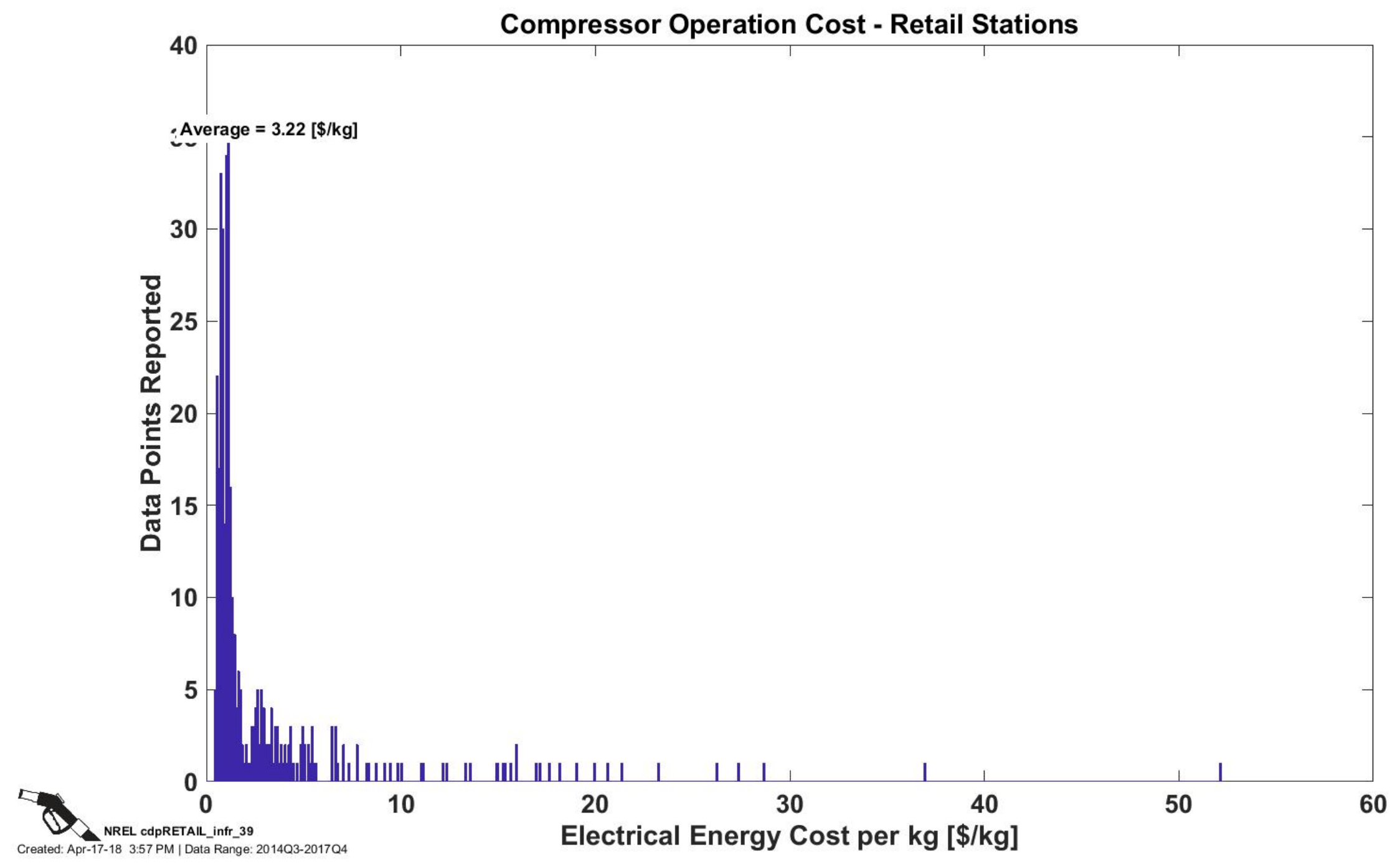




\section{CDP-INFR-40 \\ Station Cost by Daily Capacity}

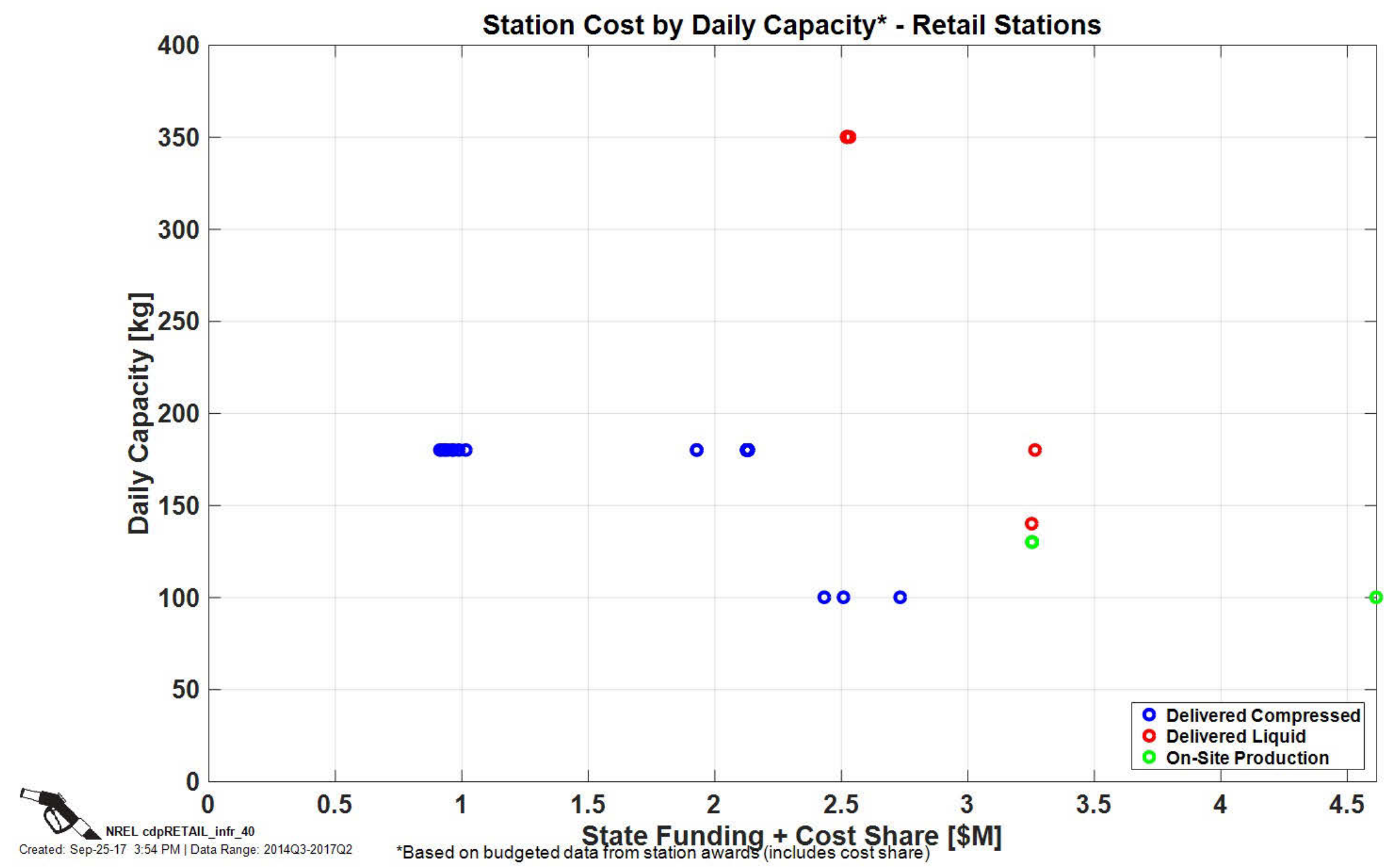




\section{CDP-INFR-41 Average Station Cost by Category}

\section{Average Station Cost by Category - Retail Stations}

\section{Budget Amounts* (Avg Total $=\$ 2.17 \mathrm{M}), 43$ Stations}
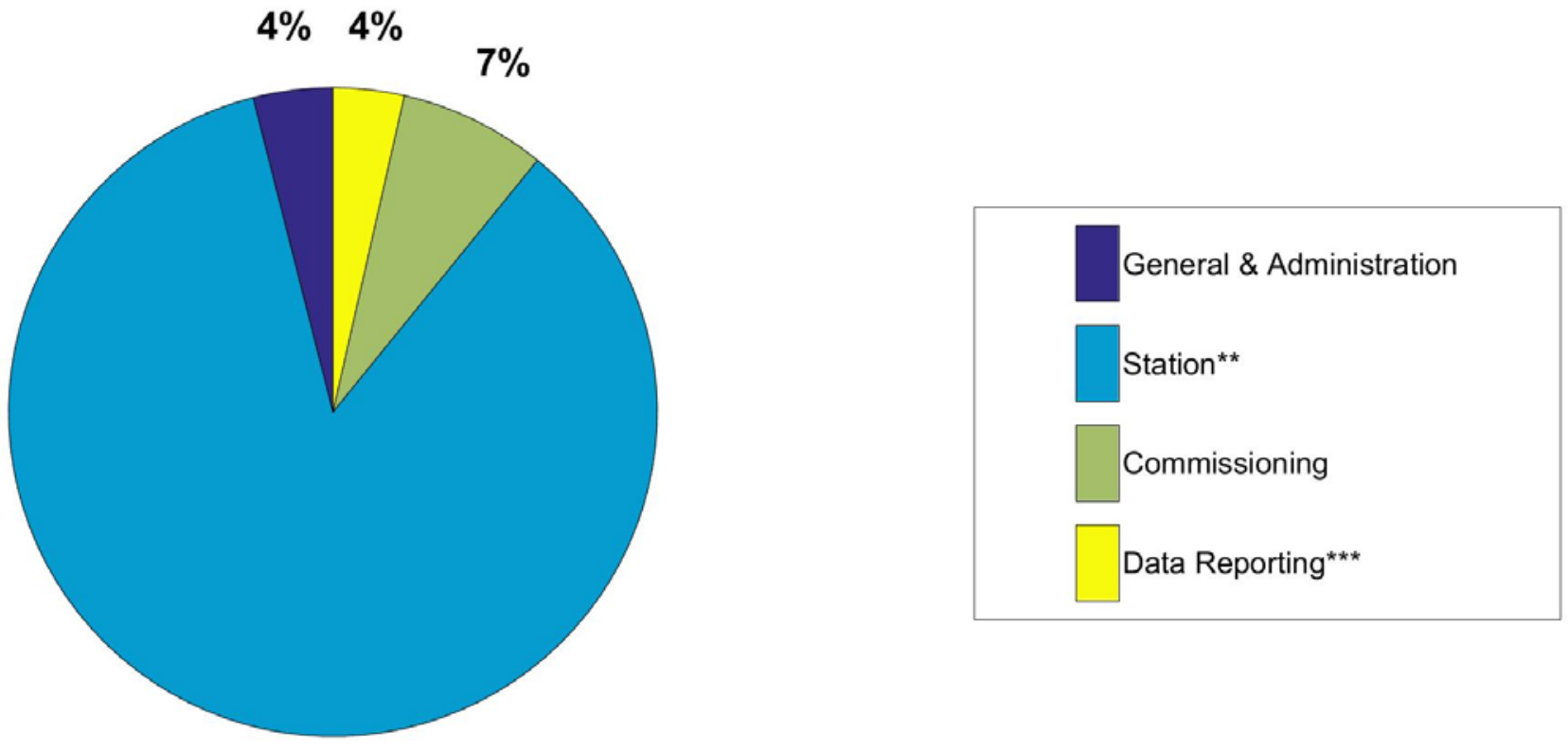

$85 \%$

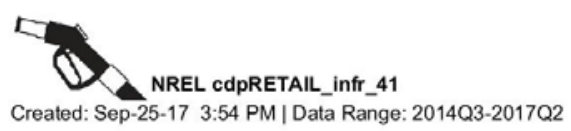

*Based on budgeted data from station awards (includes cost share)

${ }^{\star *}$ Station includes: Hydrogen Equipment and Station Engineering, Design, Fabrication, Procurement, Site Preparation, Installation, and Construction

${ }^{\star \star *}$ Data Reporting includes quarterly reporting on performance, operation and maintenance 


\section{CDP-INFR-42 Station Cost}

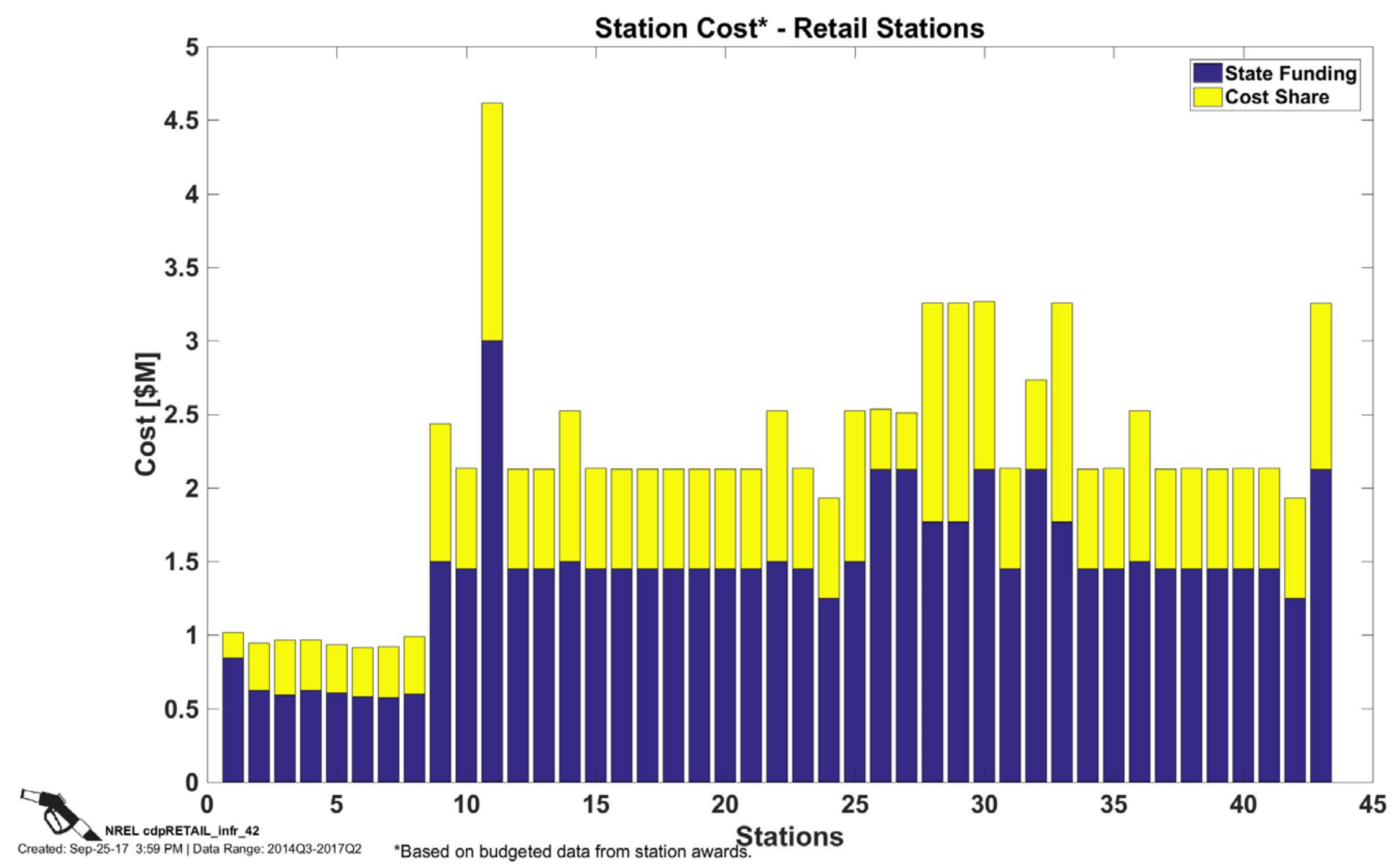




\section{CDP-INFR-43 \\ Station Cost by Type}

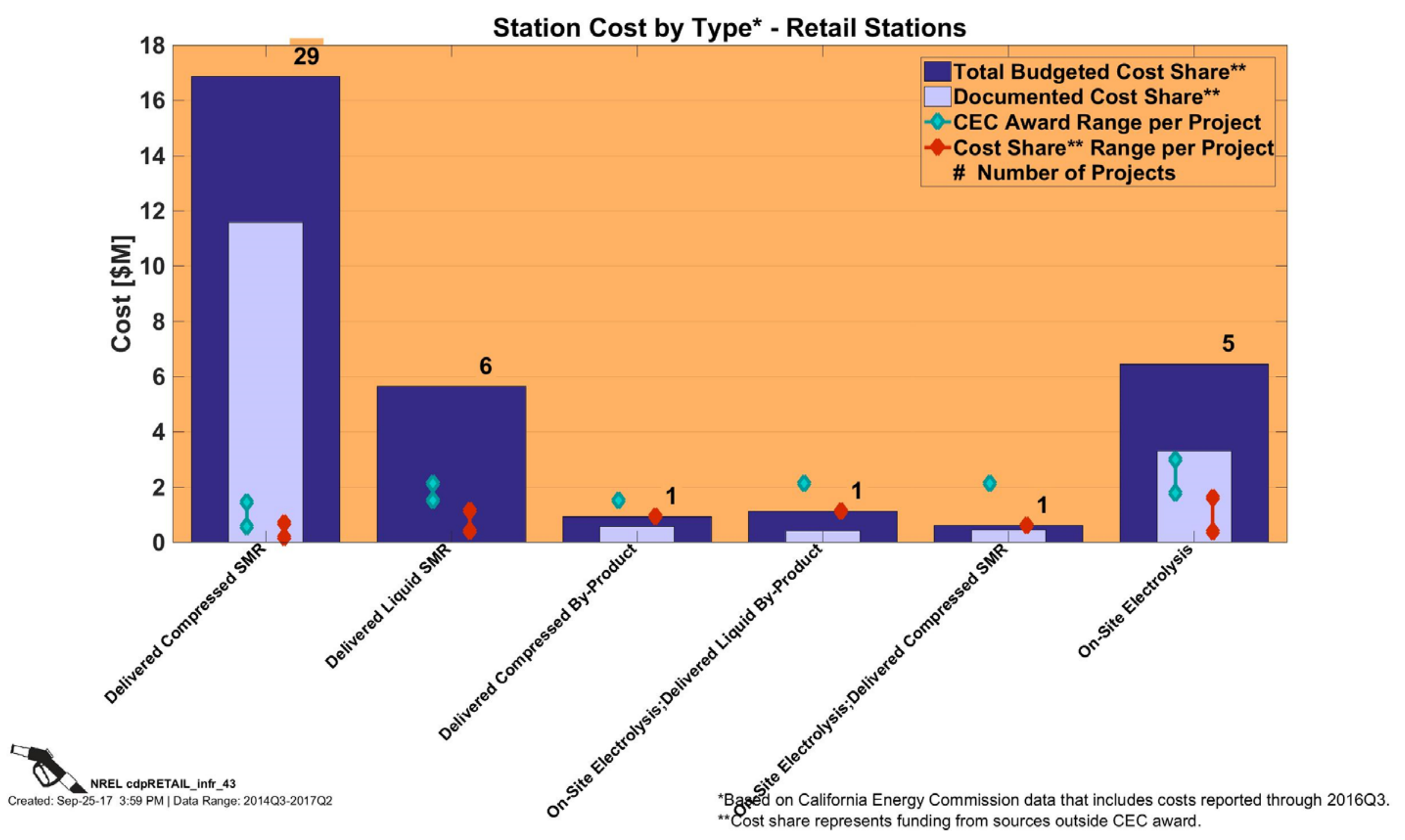




\section{CDP-INFR-53}

\section{Maintenance Cost per kg of Hydrogen Dispensed}

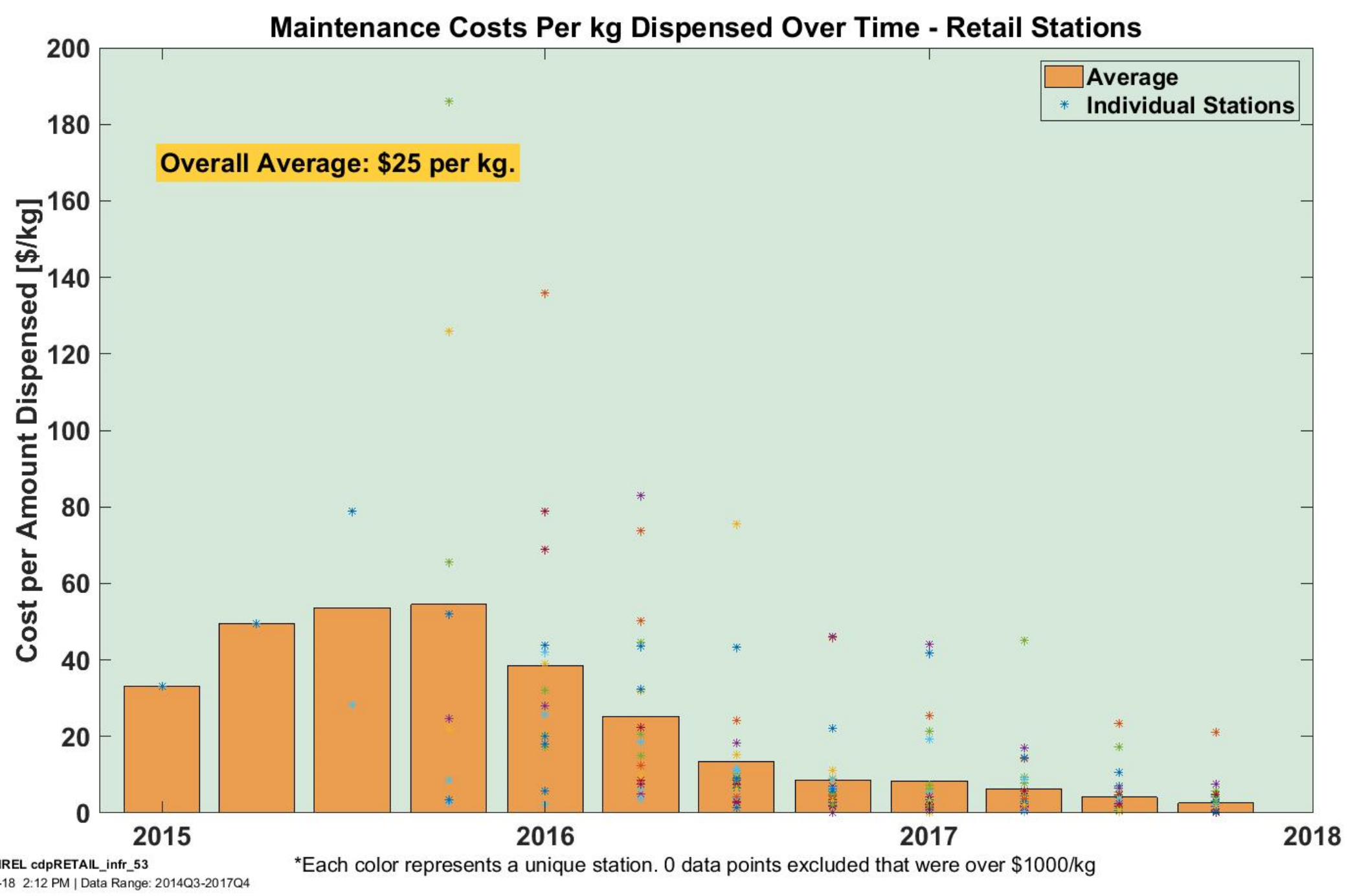




\section{CDP-INFR-73}

\section{Histogram of Monthly Maintenance Costs}

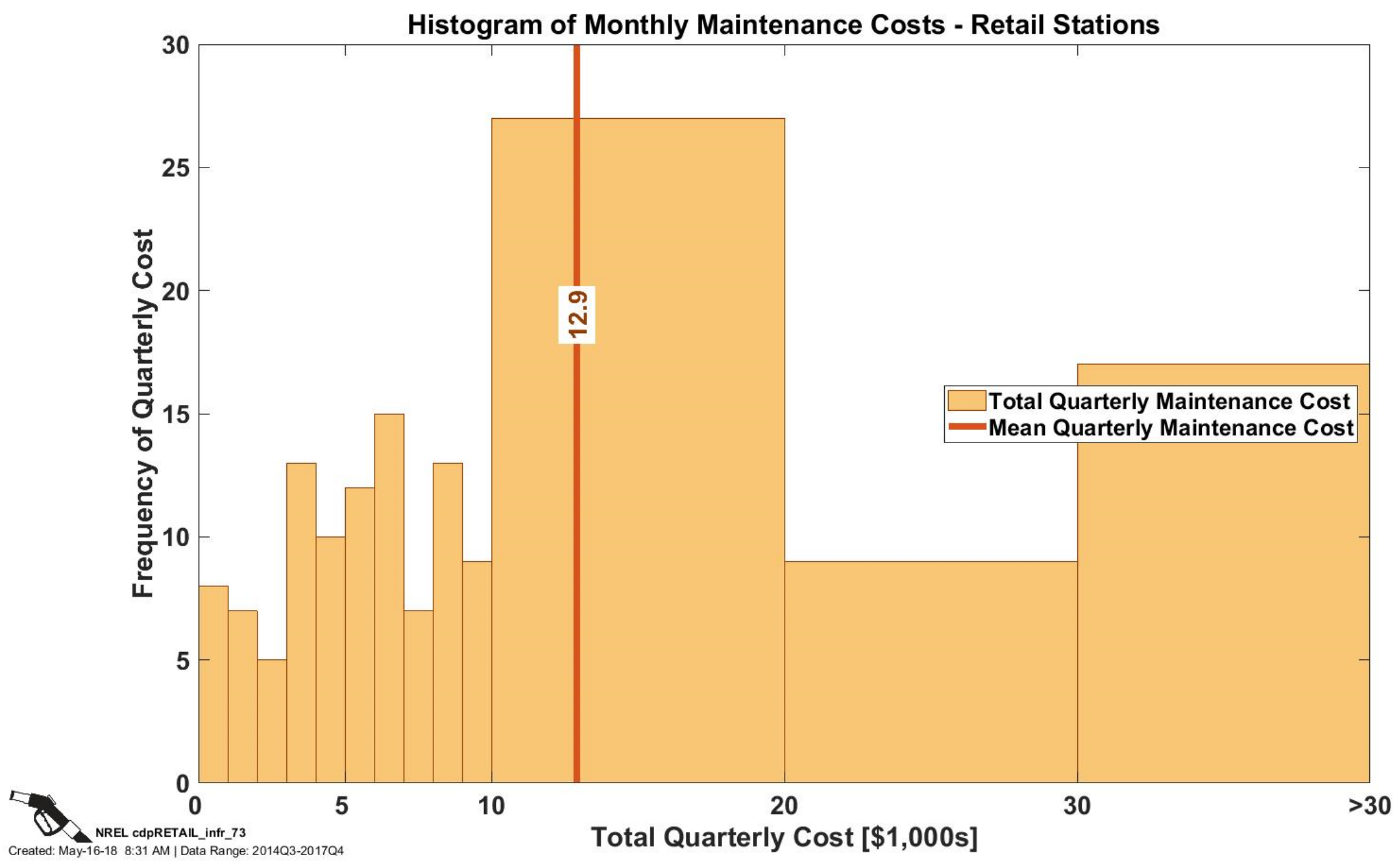




\section{CDP-INFR-89 \\ Hydrogen Price by Quarter}

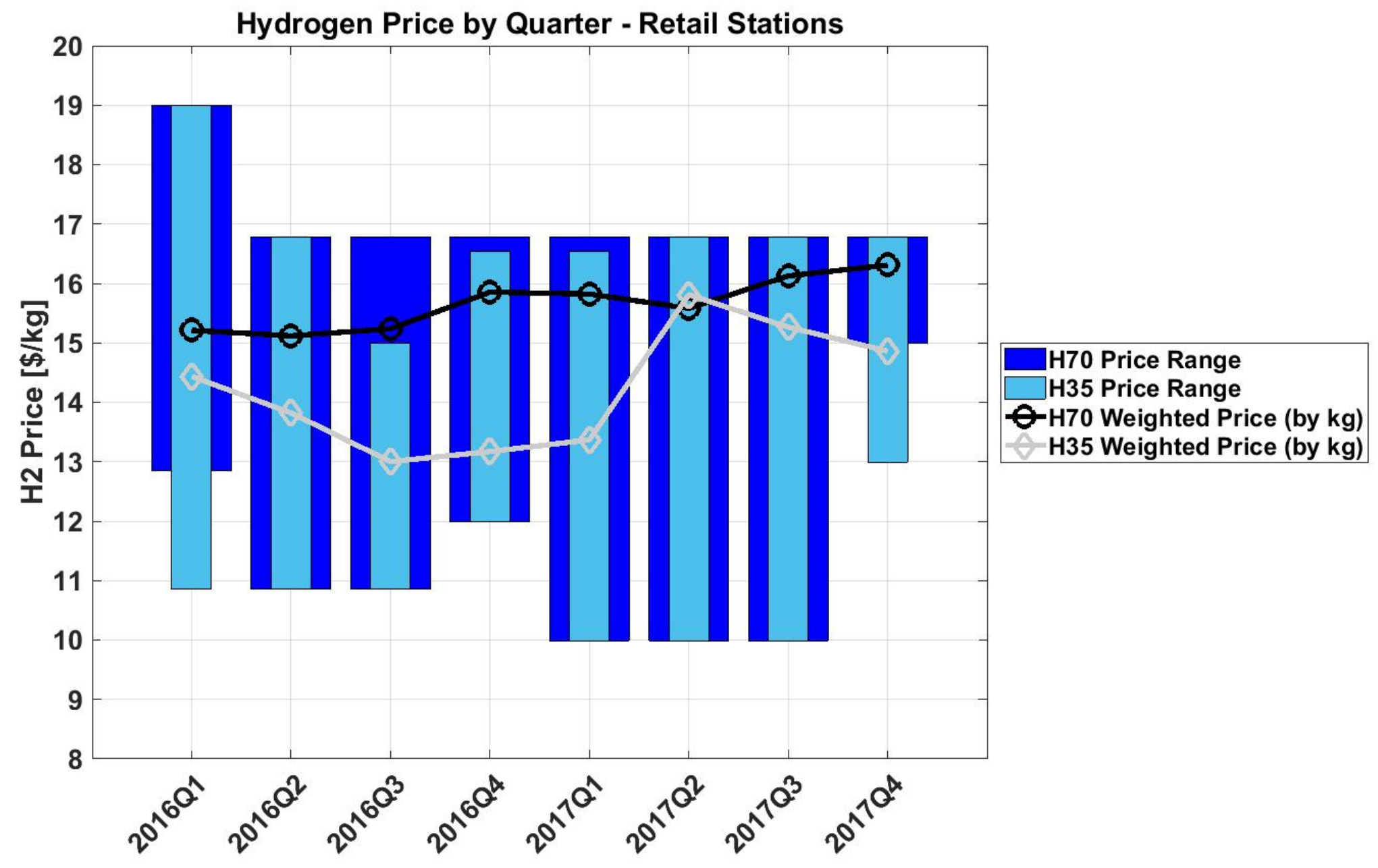

NREL cdpRETAIL_infr_89

Created: May-05-18 5:28 AM | Data Range: 2014Q3-2017Q4 
Utilization 


\section{CDP-INFR-05 \\ Dispensed Hydrogen per Day of Week}

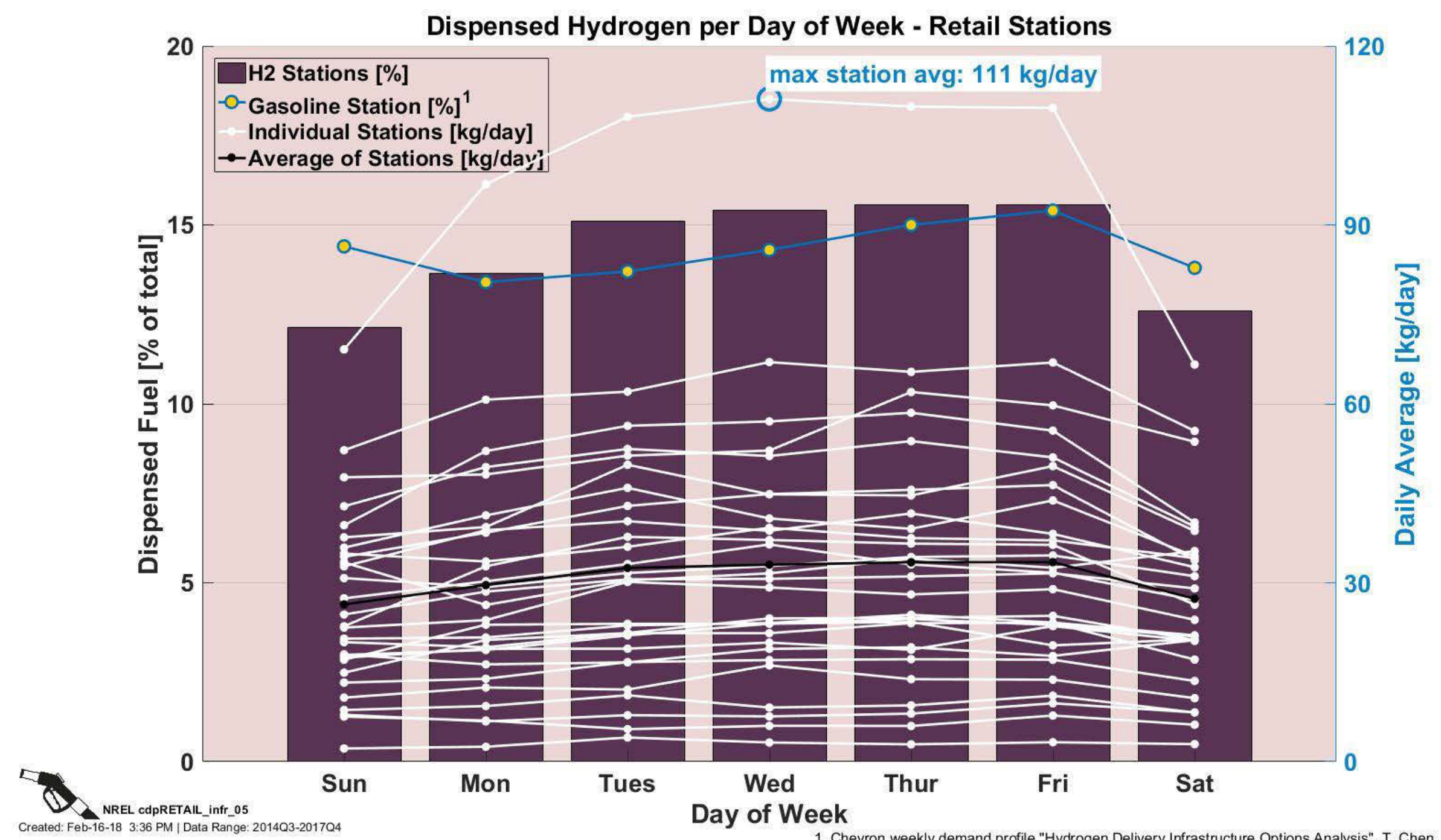




\section{CDP-INFR-06 \\ Station Capacity Utilization}

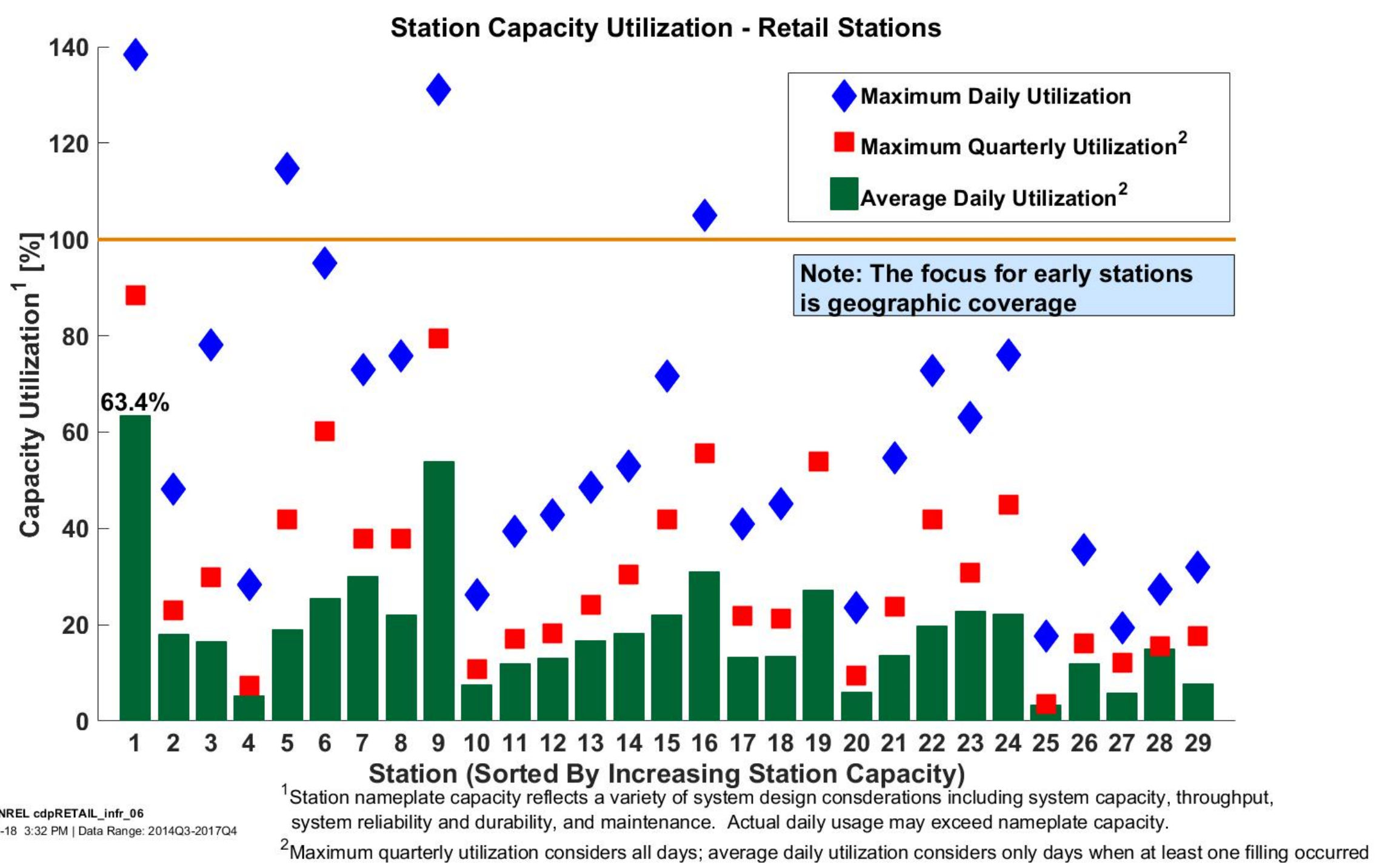




\section{CDP-INFR-07 Station Usage}

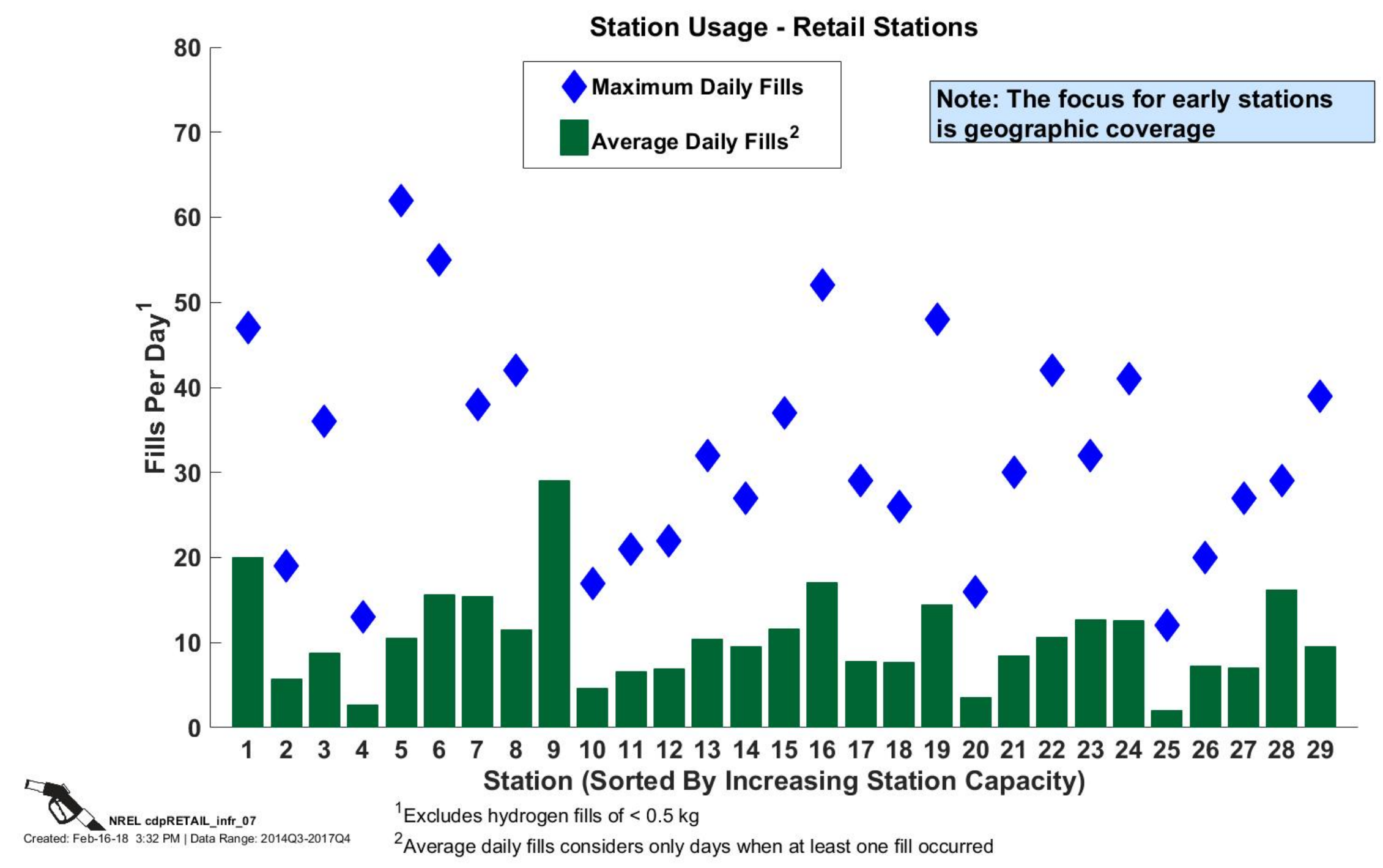




\section{CDP-INFR-19 \\ Hydrogen Dispensed by Month}

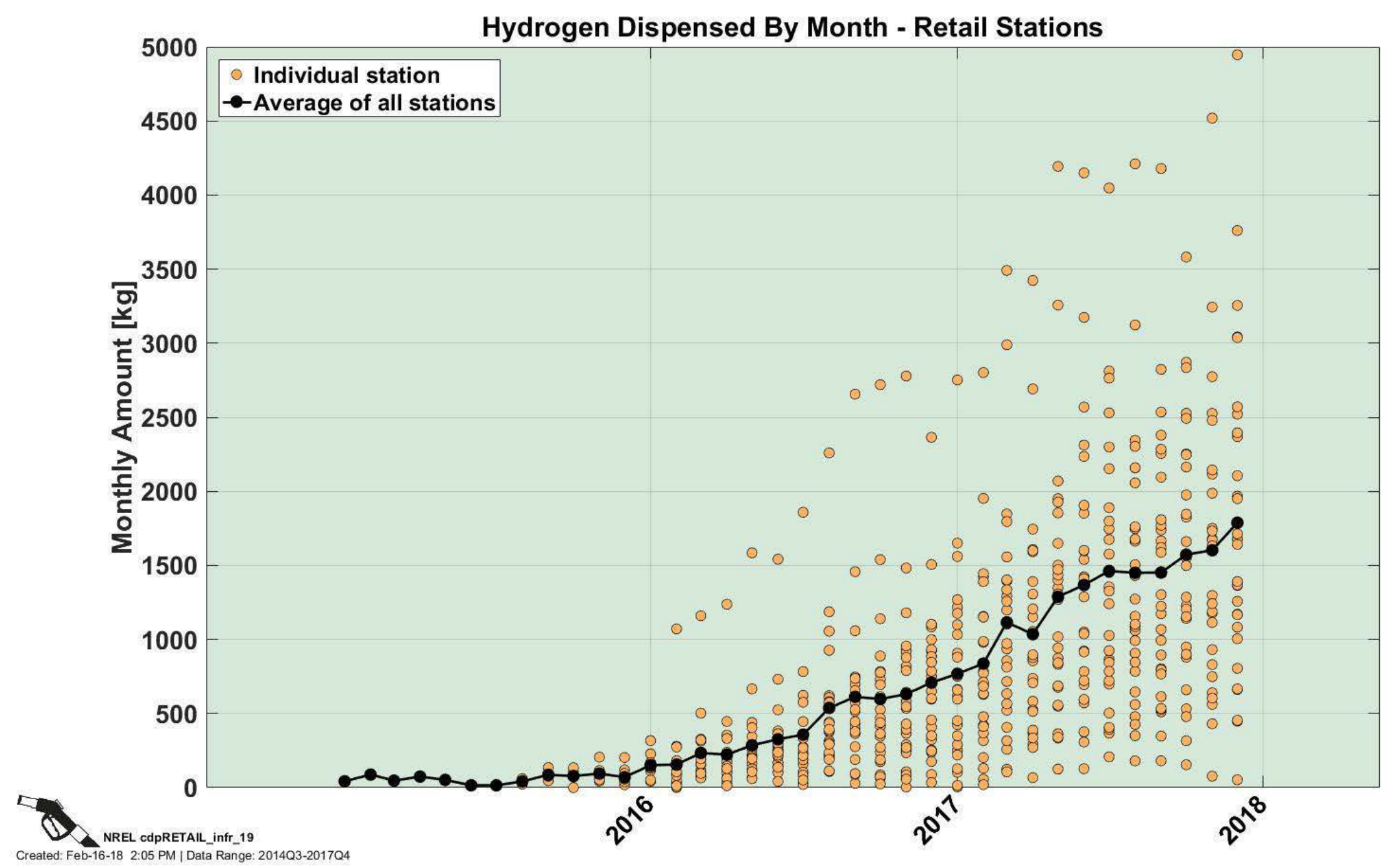




\section{Number of Fills by Month}

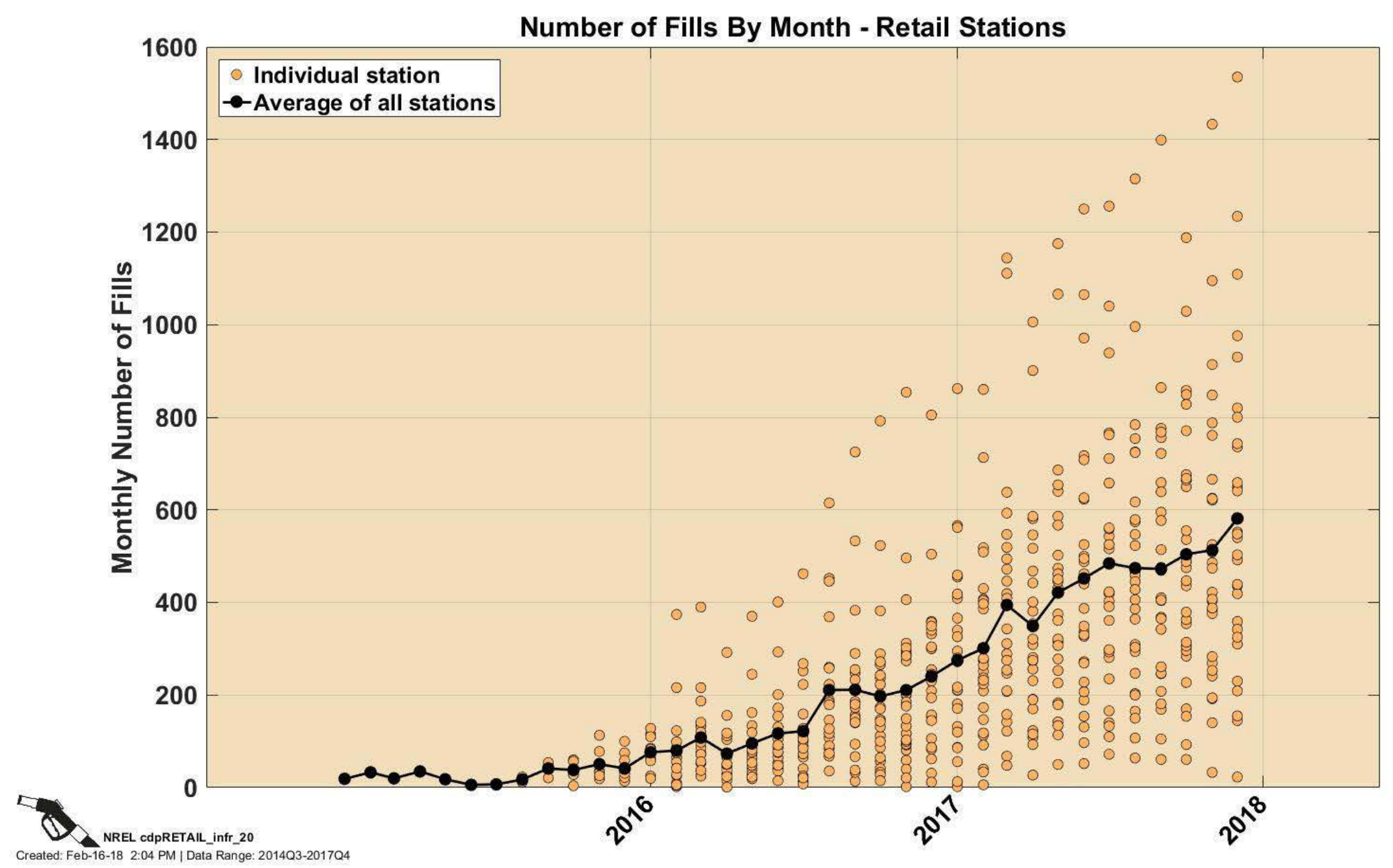




\section{Station Capacity Utilization Trends by Quarter}

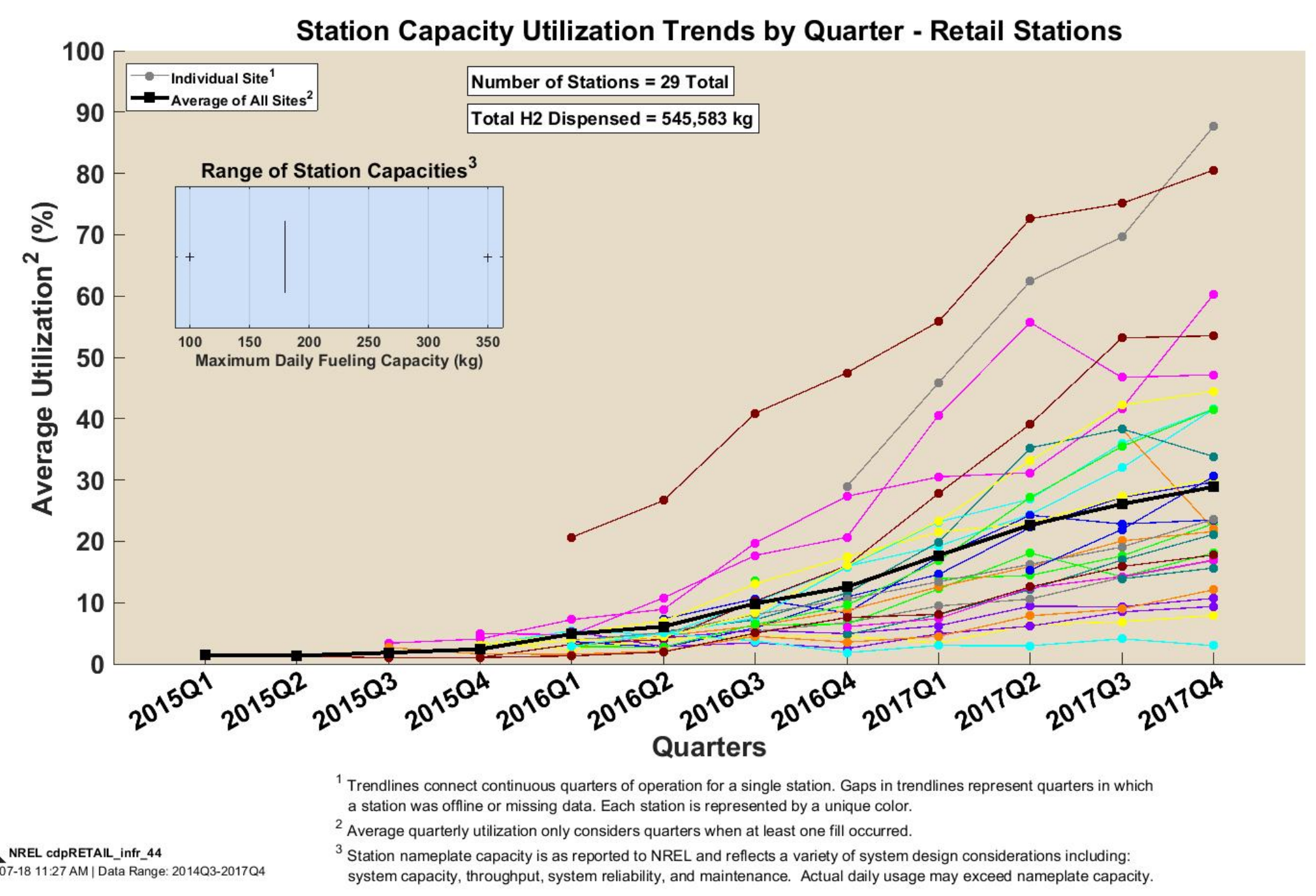




\section{CDP-INFR-45}

\section{Station Amount Dispensed by Quarter}

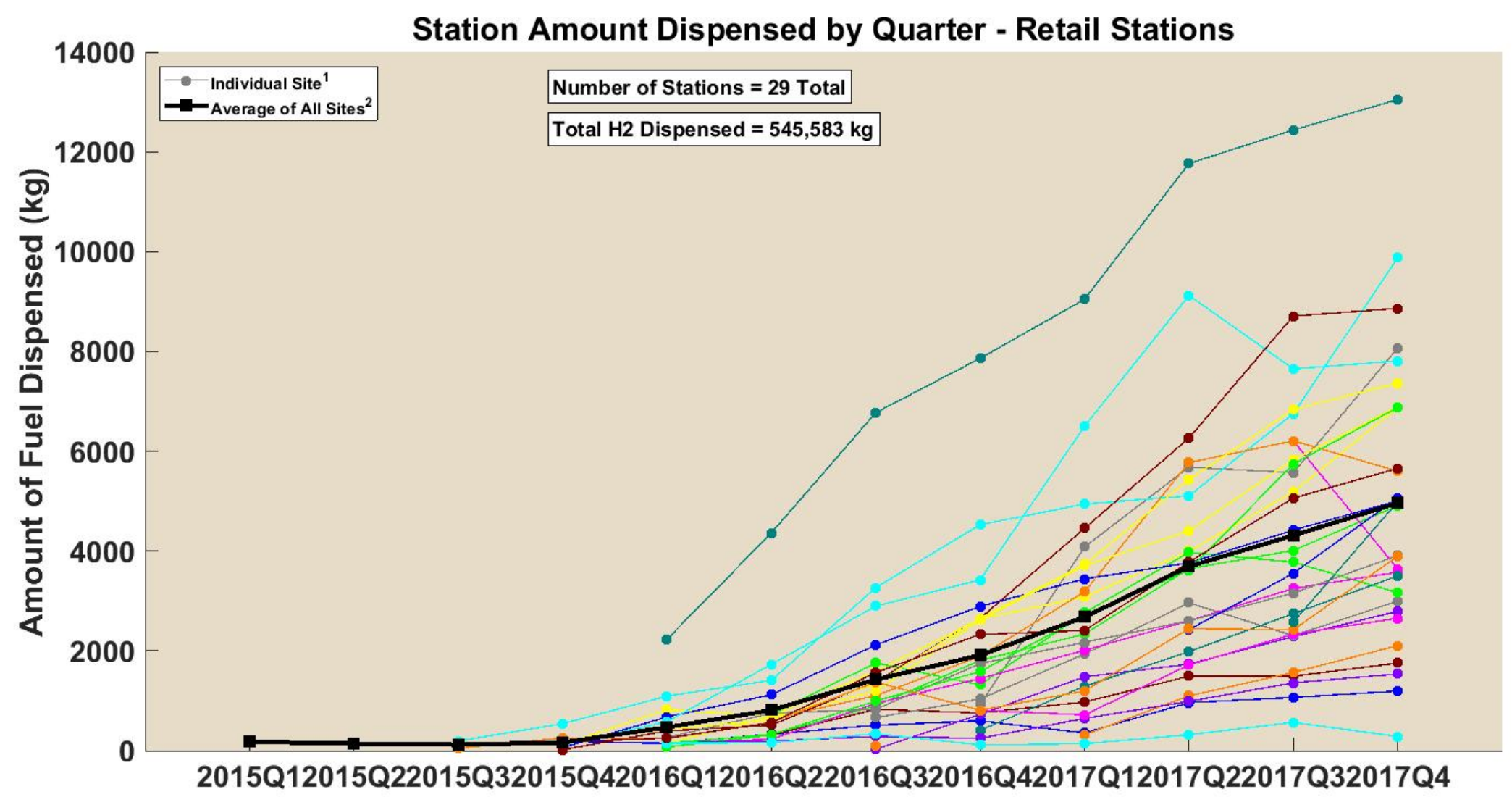

${ }^{2}$ Average quarterly amount only considers quarters when at least one fill occurred. 


\section{CDP-INFR-46 \\ Days with Fills by Quarter}

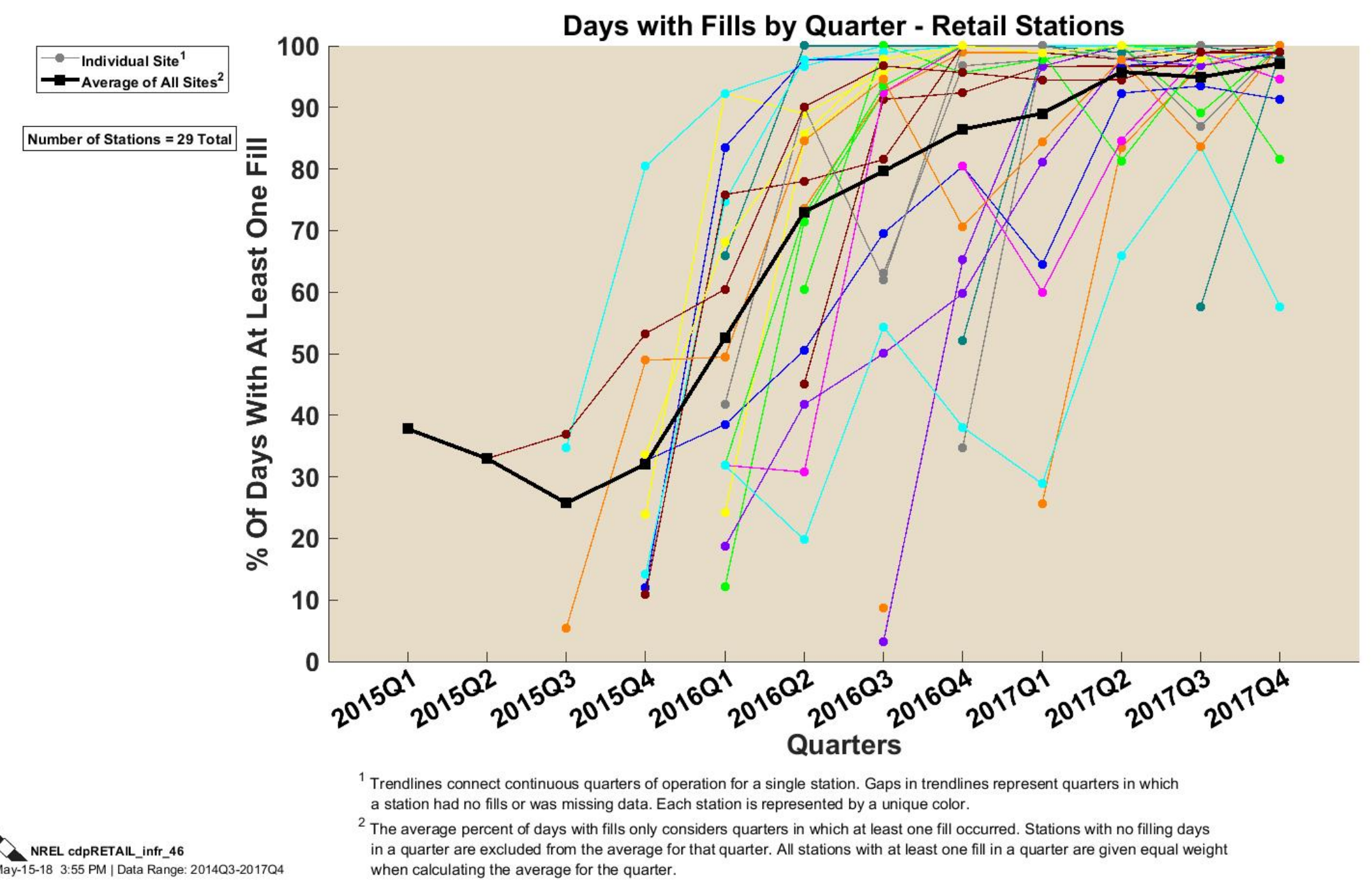




\section{Summary of Station Usage Statistics}

\section{Summary of Station Usage Statistics - Retail Stations ${ }^{4}$}
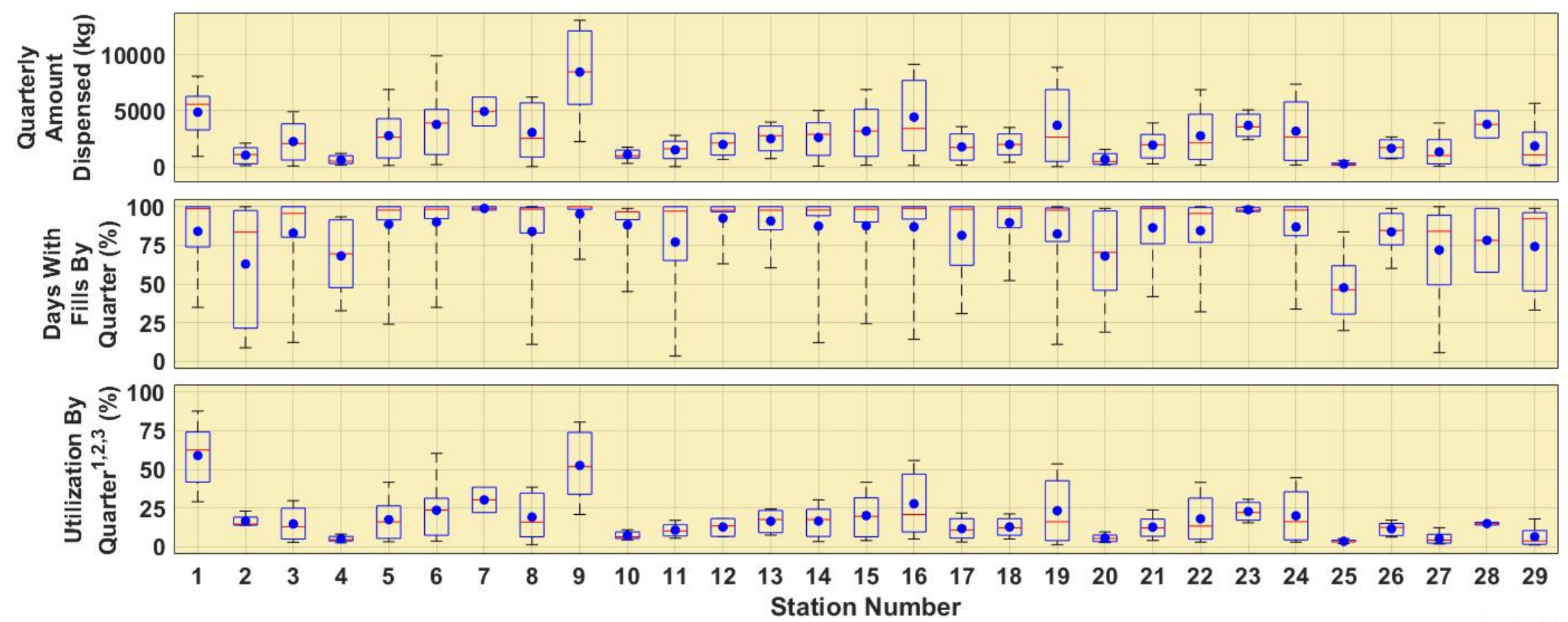

${ }^{1}$ Station nameplate capacity is as reported to NREL and reflects a variety of system design considerations including: system capacity, throughput, system reliability, and maintenance. Actual daily usage may exceed nameplate capacity.

${ }^{2}$ Average quarterly utilization only considers days when at least one fill occured.

${ }^{3}$ Utilization is calculated by dividing the quarterly amount dispensed by the stations nameplate capacity.

${ }^{4}$ Only quarters with fills are included.

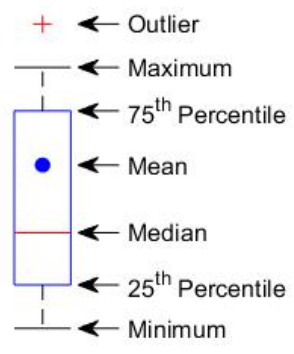




\section{CDP-INFR-80}

\section{Daily Fueling Amounts by Station}
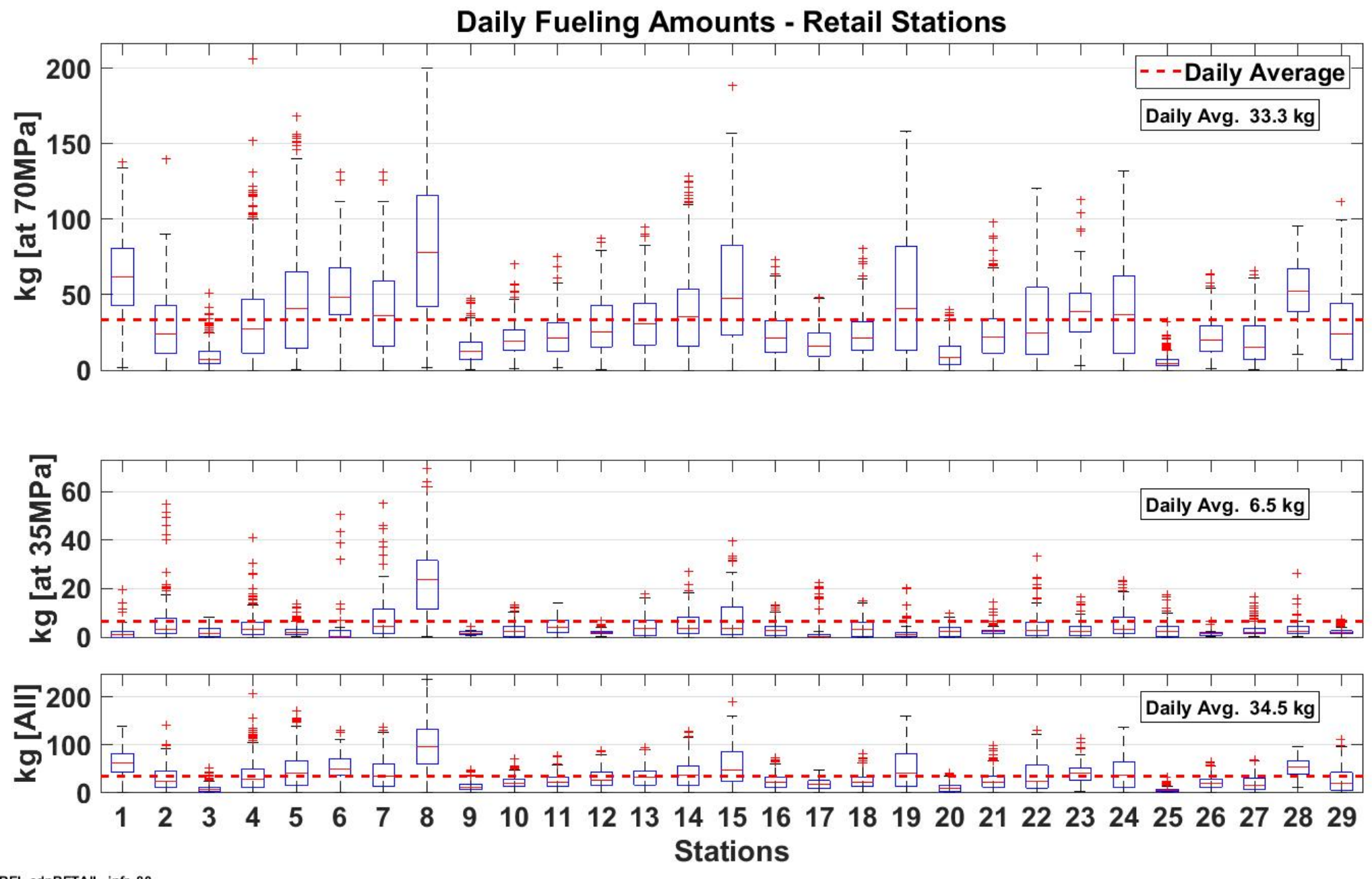

NREL CdPRETAIL_infr_80 


\section{Daily Fueling Amounts by Month}
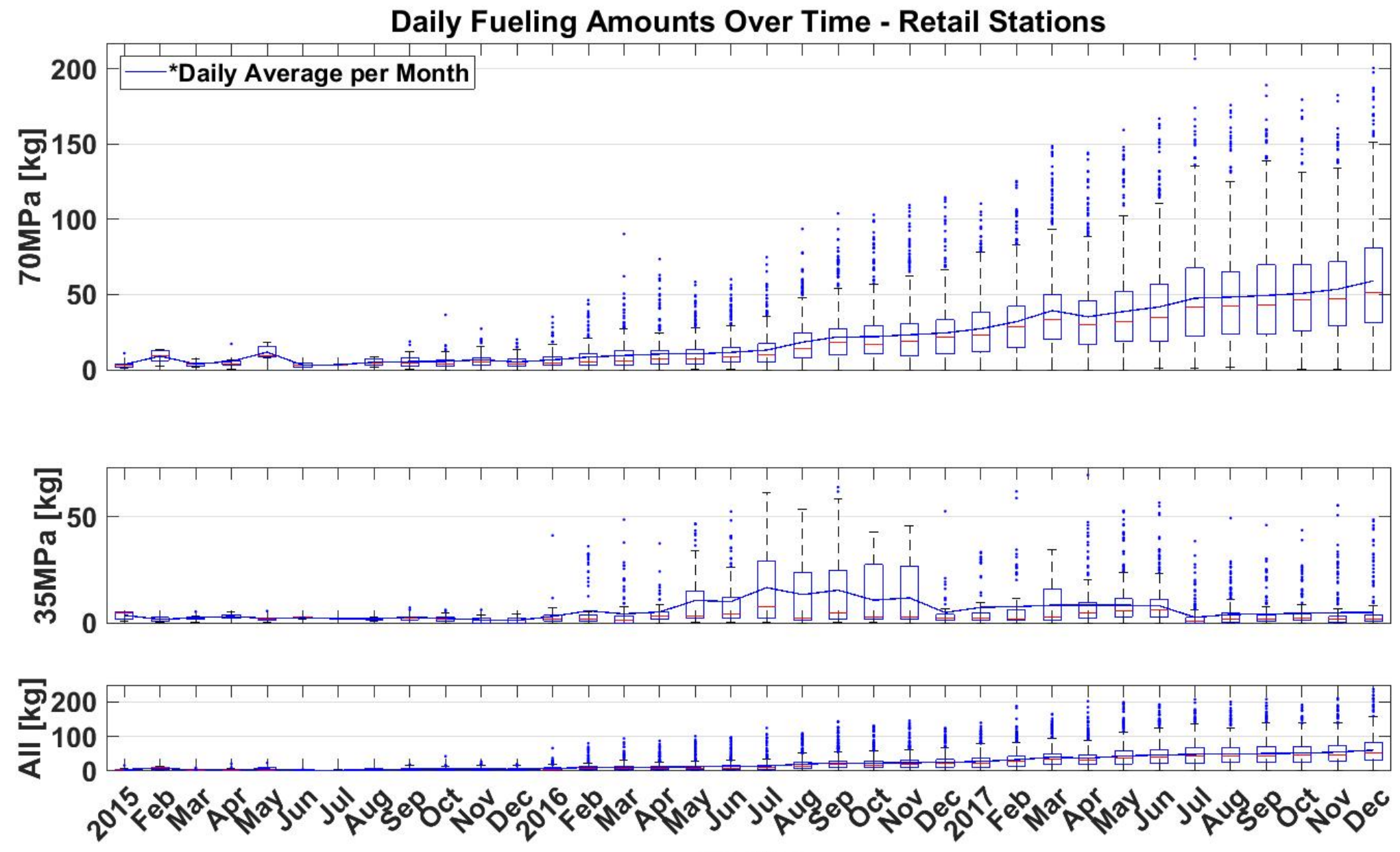

Months 
Hydrogen Quality

(20) 


\section{CDP-INFR-25 \\ Hydrogen Quality}

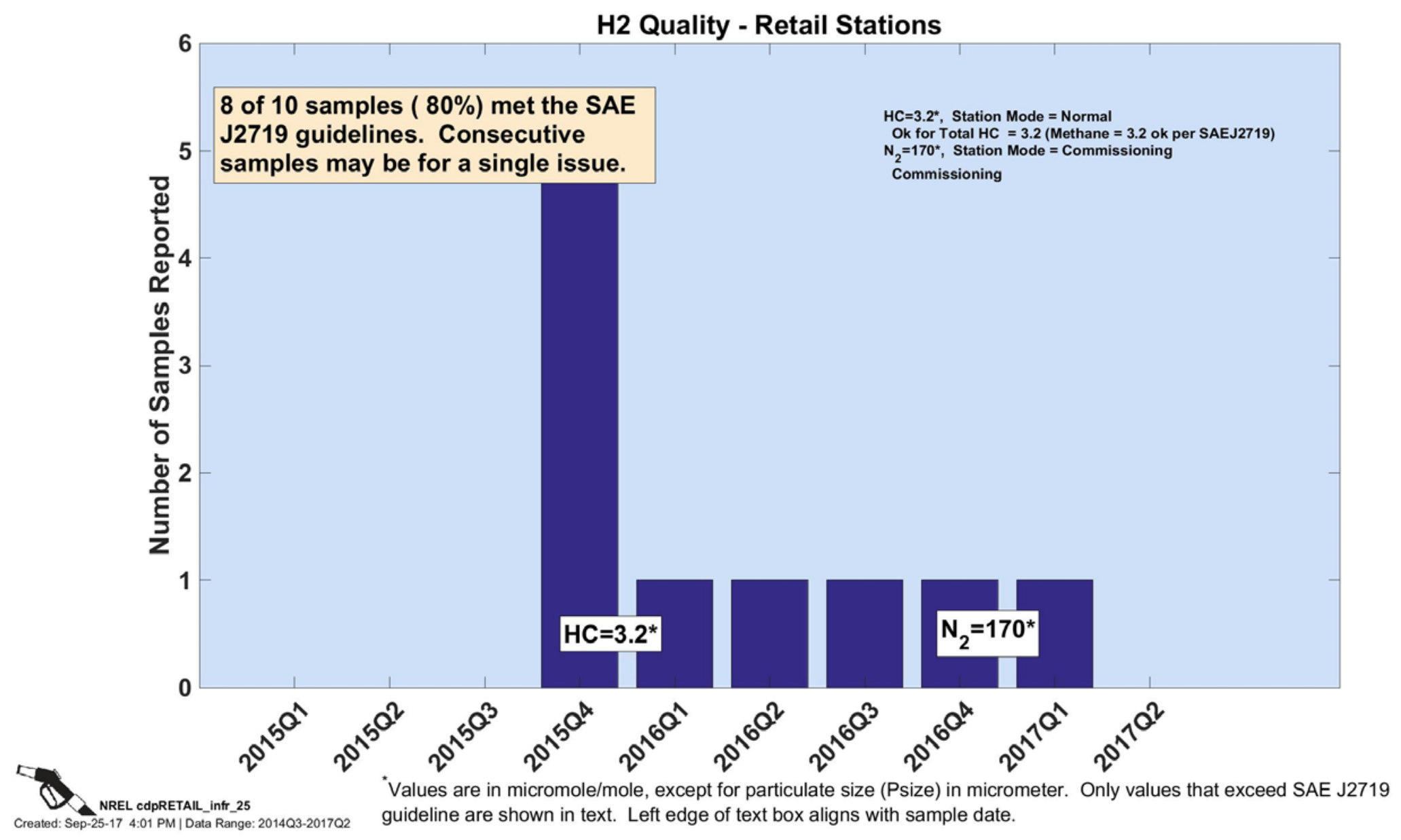




\section{Impurities-Ammonia}

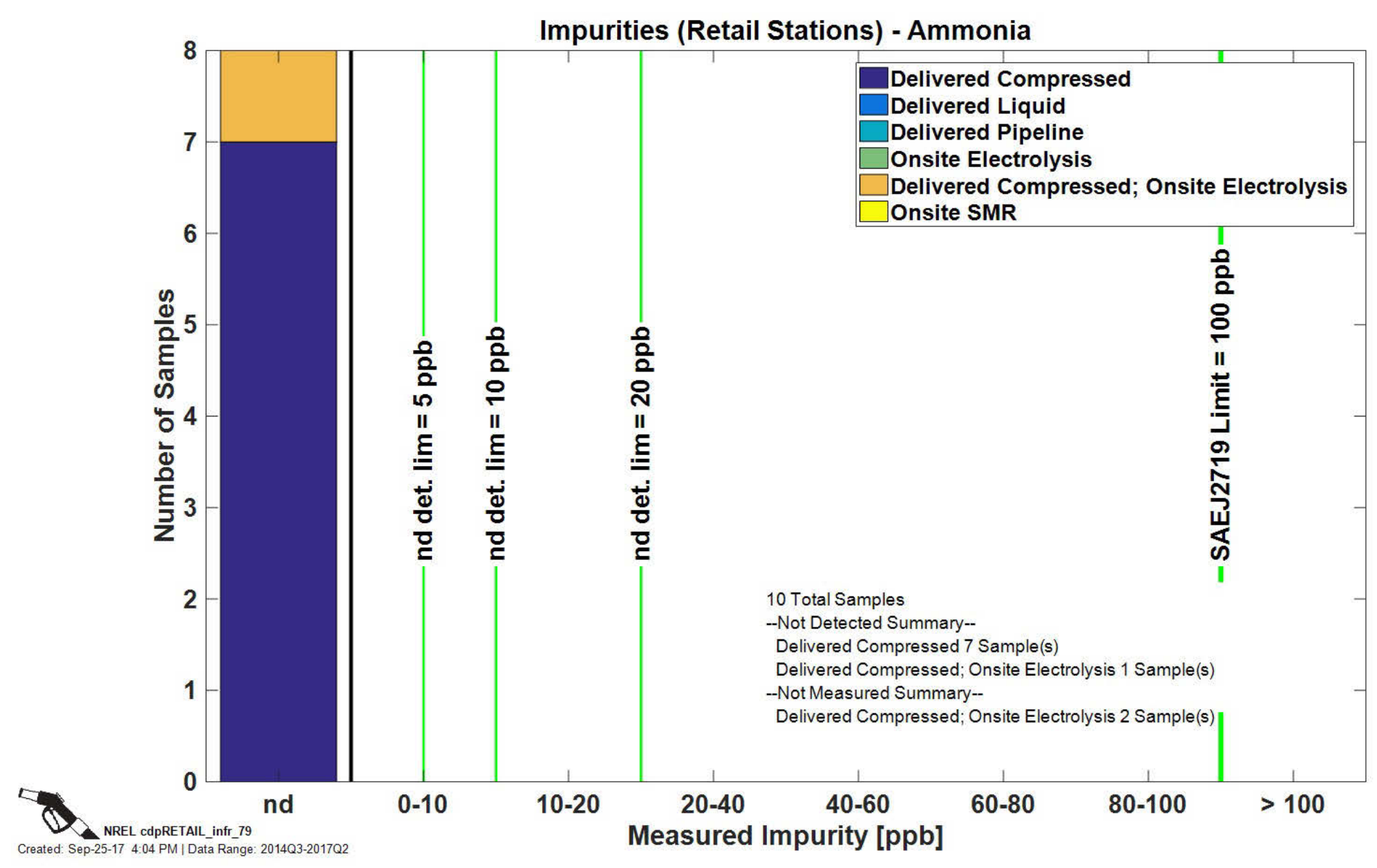




\section{CDP-INFR-79 \\ Impurities-Argon}

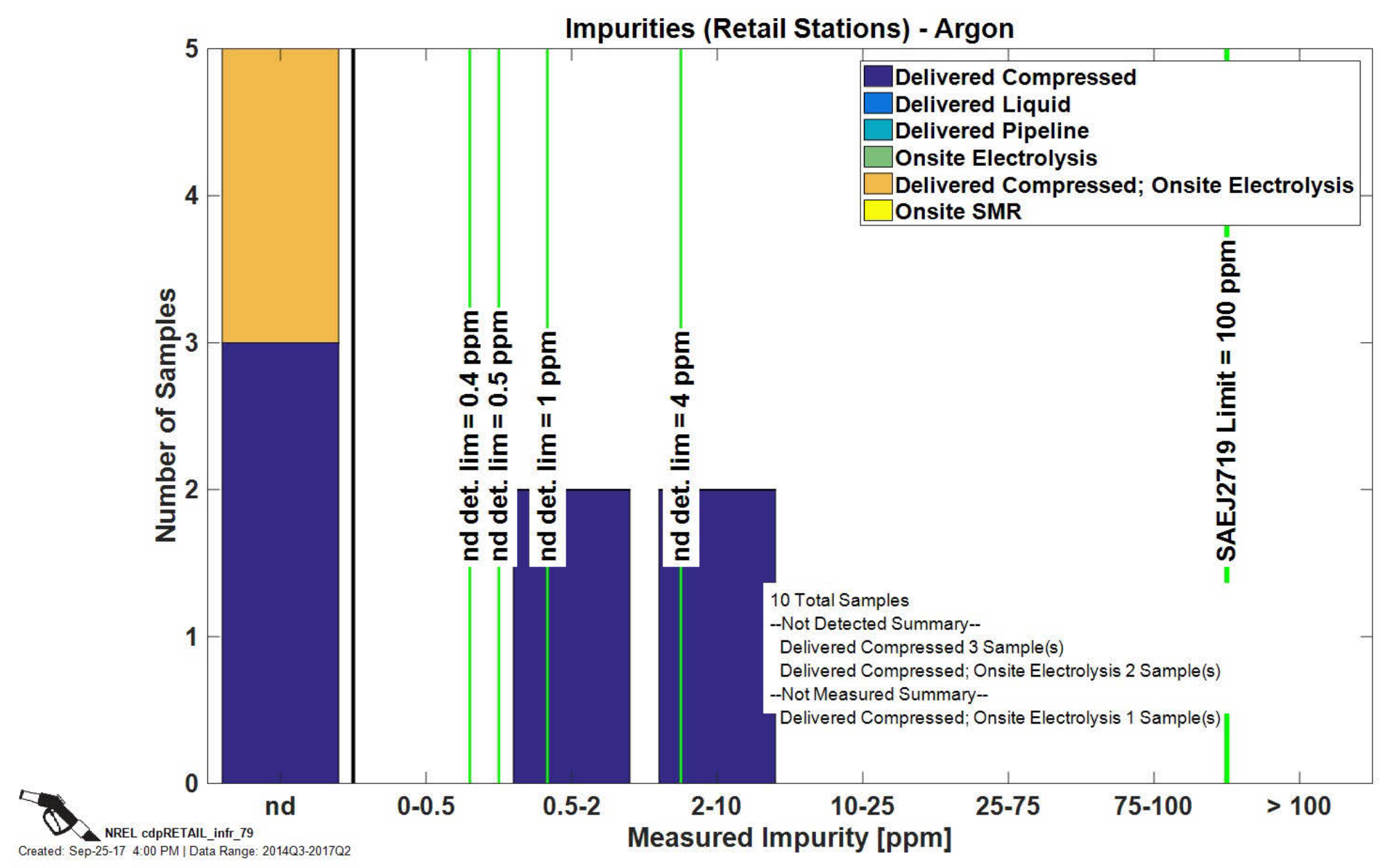




\section{CDP-INFR-79}

\section{Impurities-Carbon Dioxide}

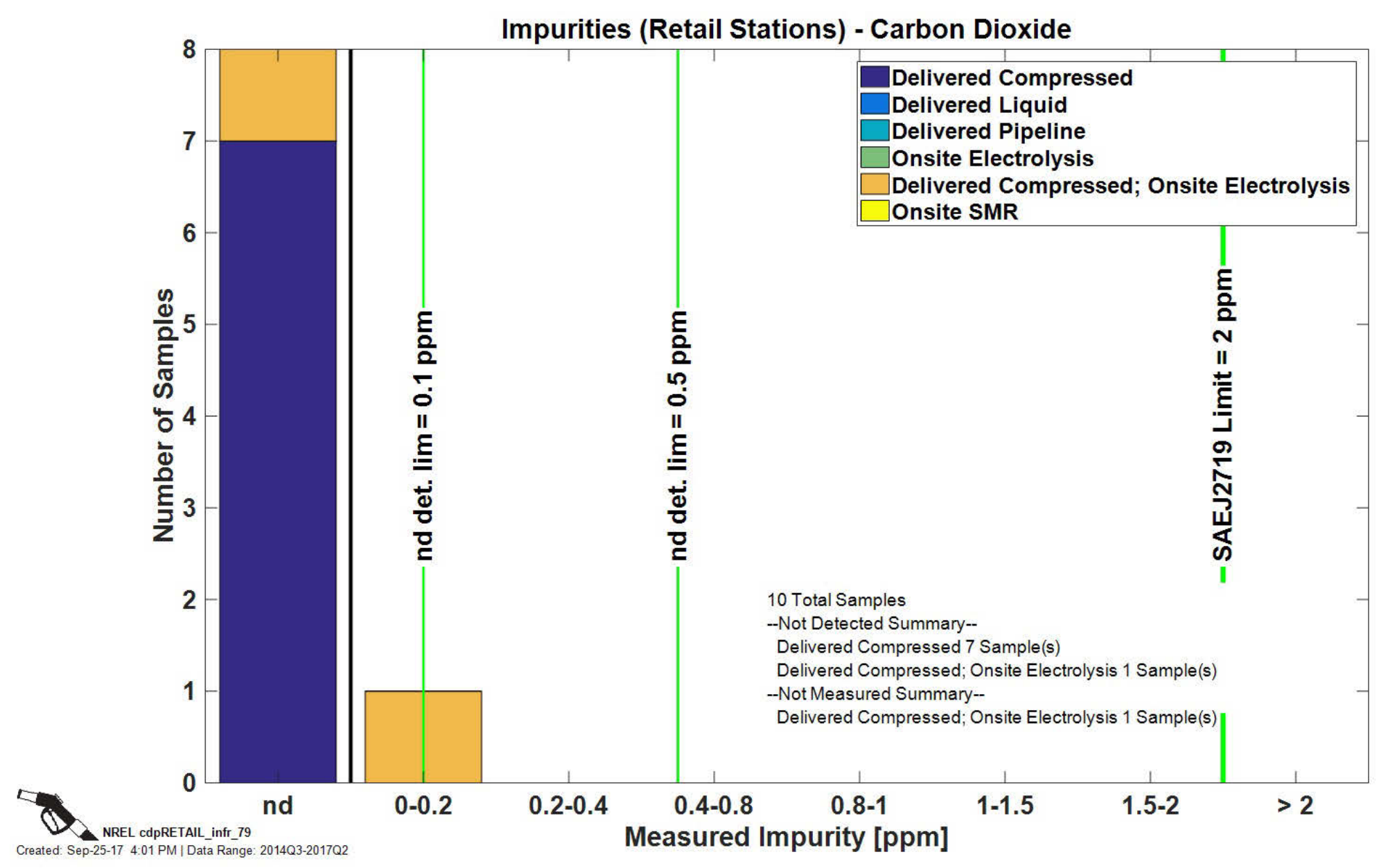




\section{CDP-INFR-79}

\section{Impurities-Carbon Monoxide}

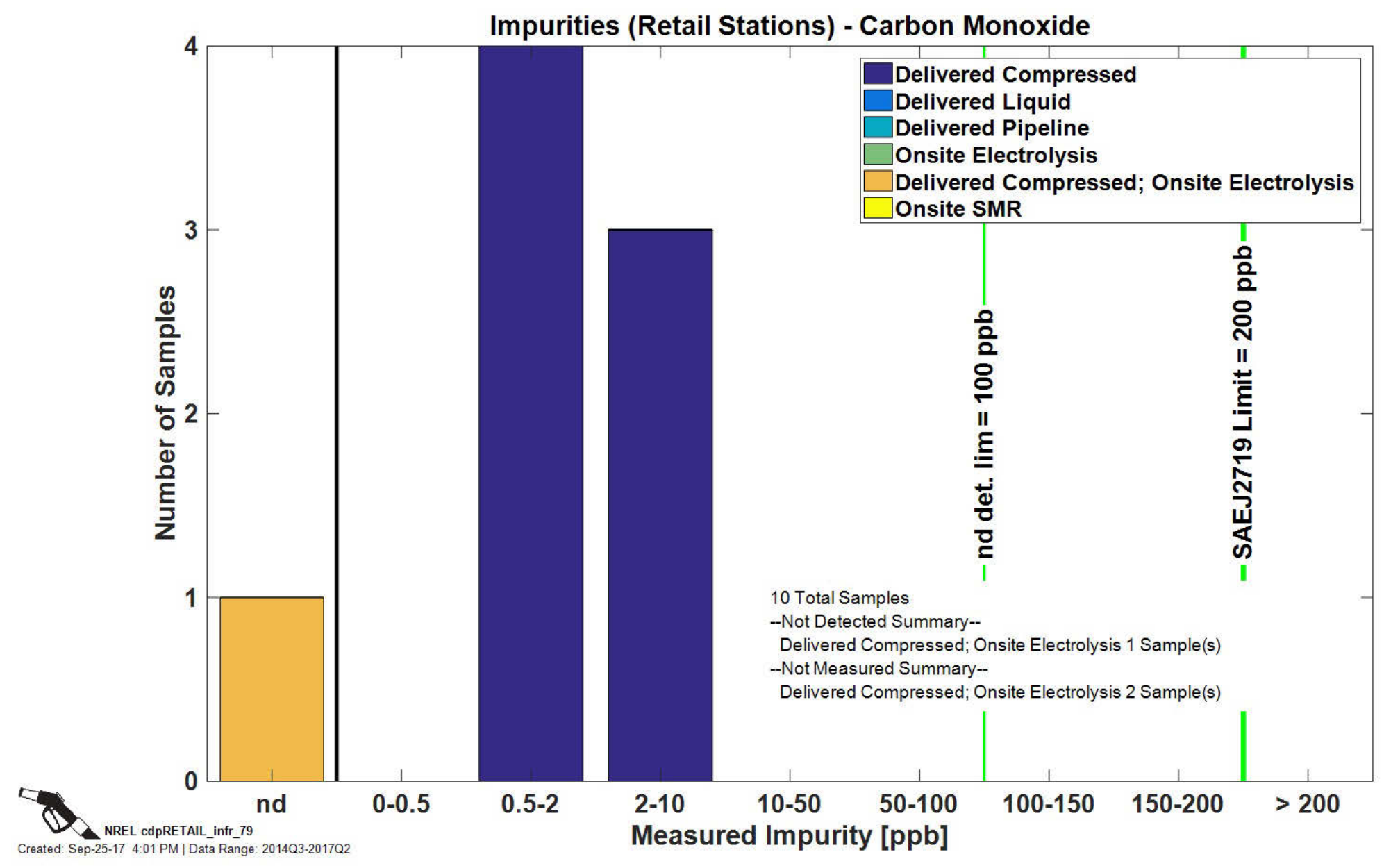




\section{CDP-INFR-79}

\section{Impurities-Formaldehyde}

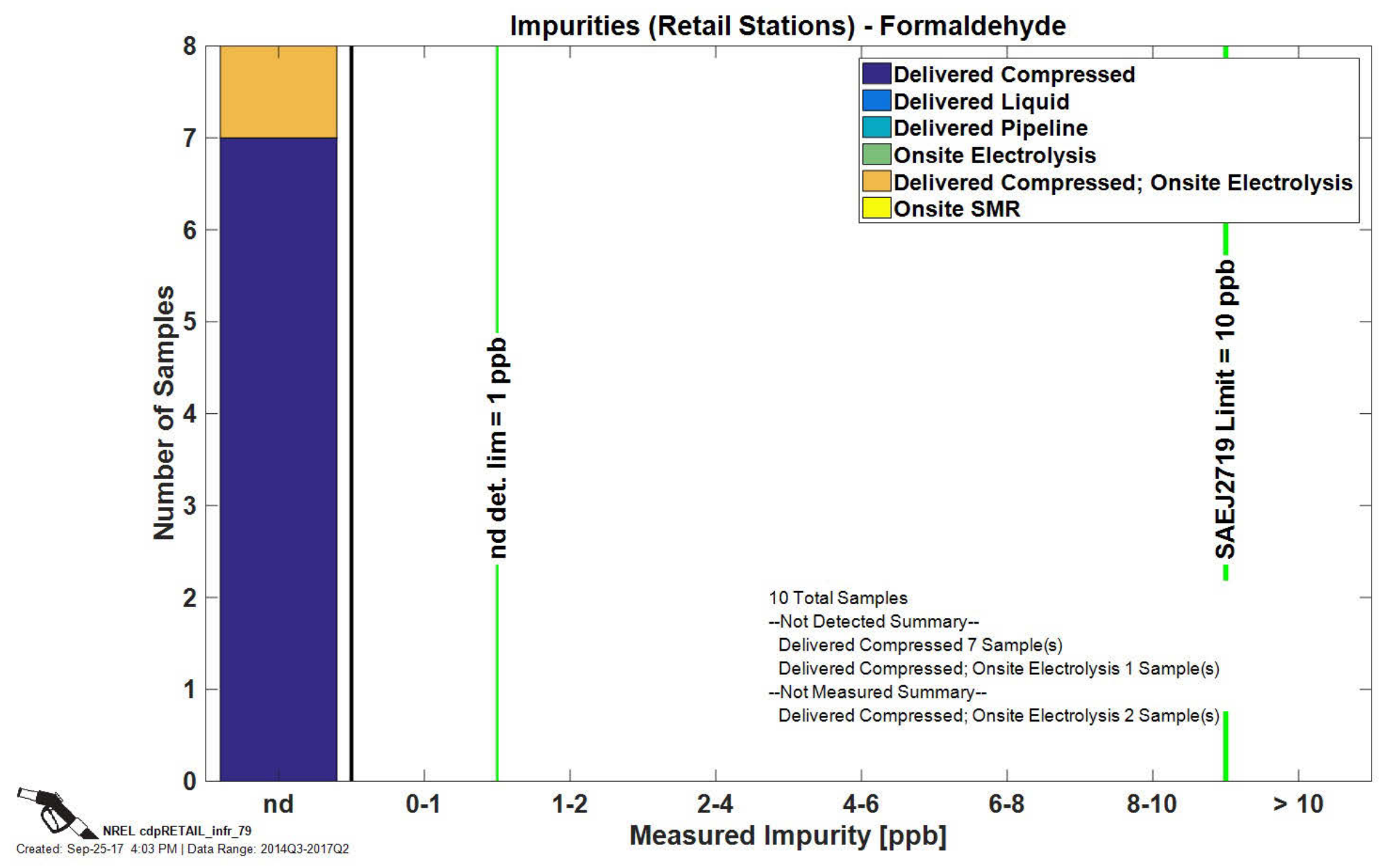




\section{CDP-INFR-79}

\section{Impurities-Formic Acid}

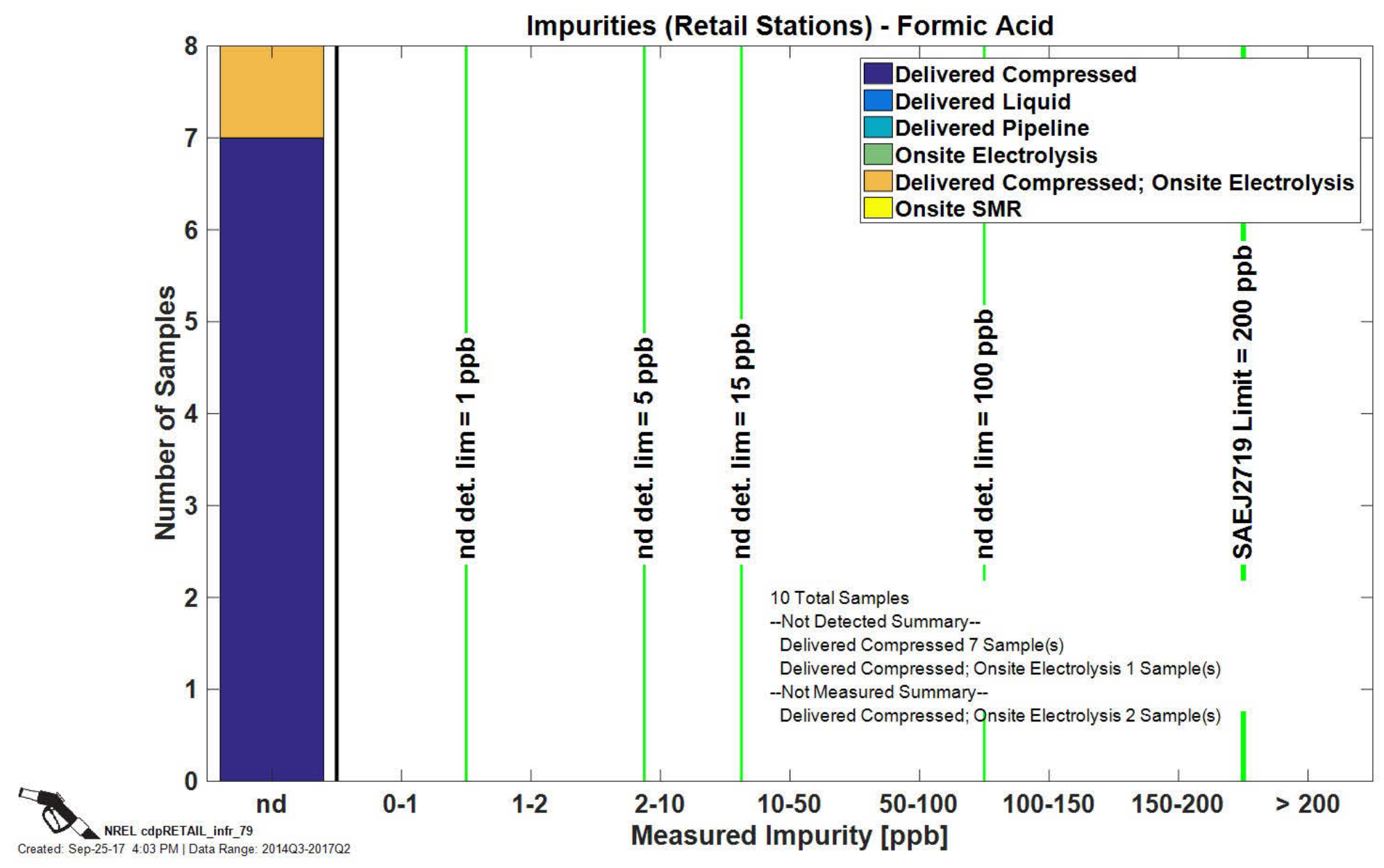




\section{CDP-INFR-79 \\ Impurities-Helium}

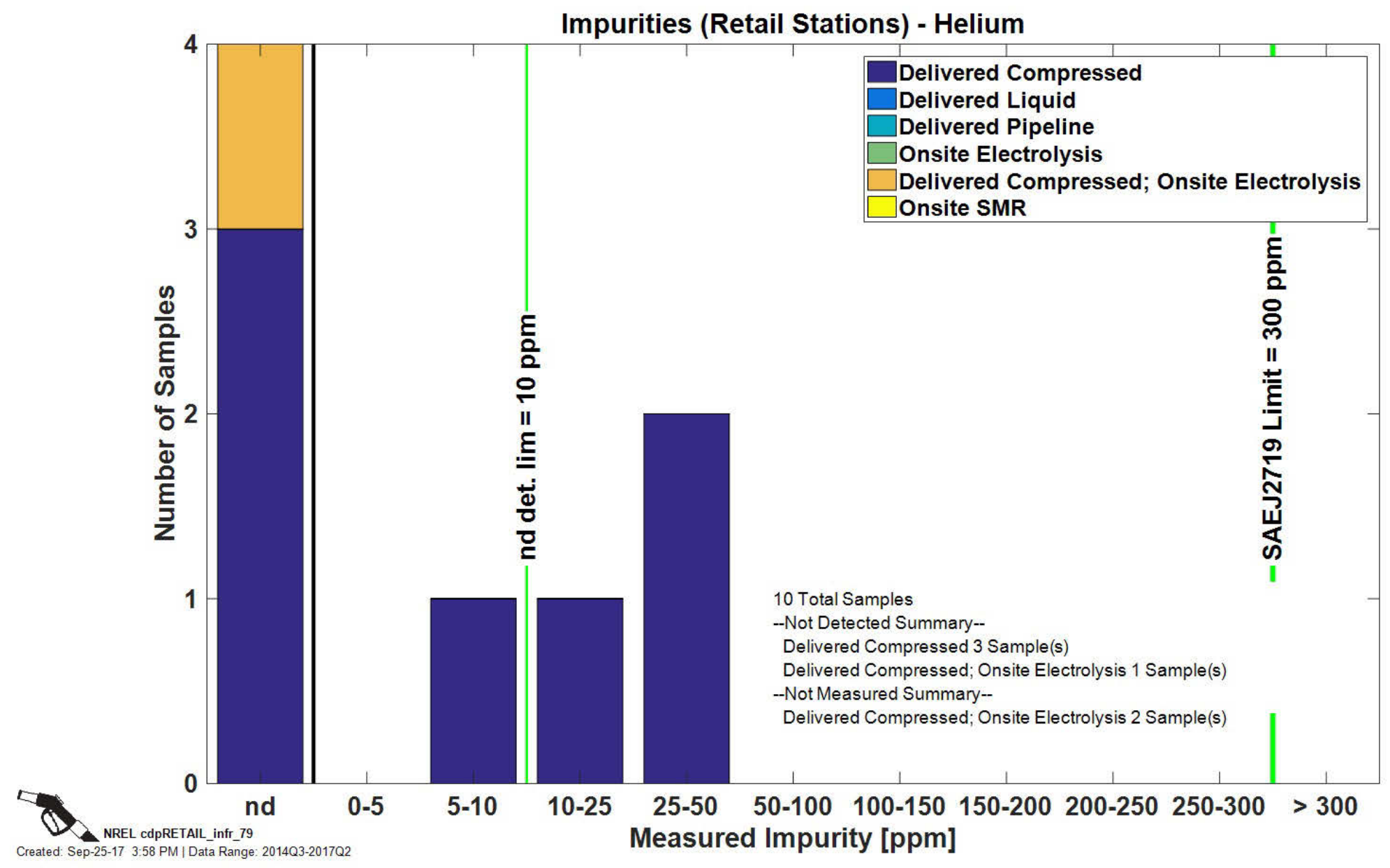




\section{CDP-INFR-79 \\ Impurities-Nitrogen}

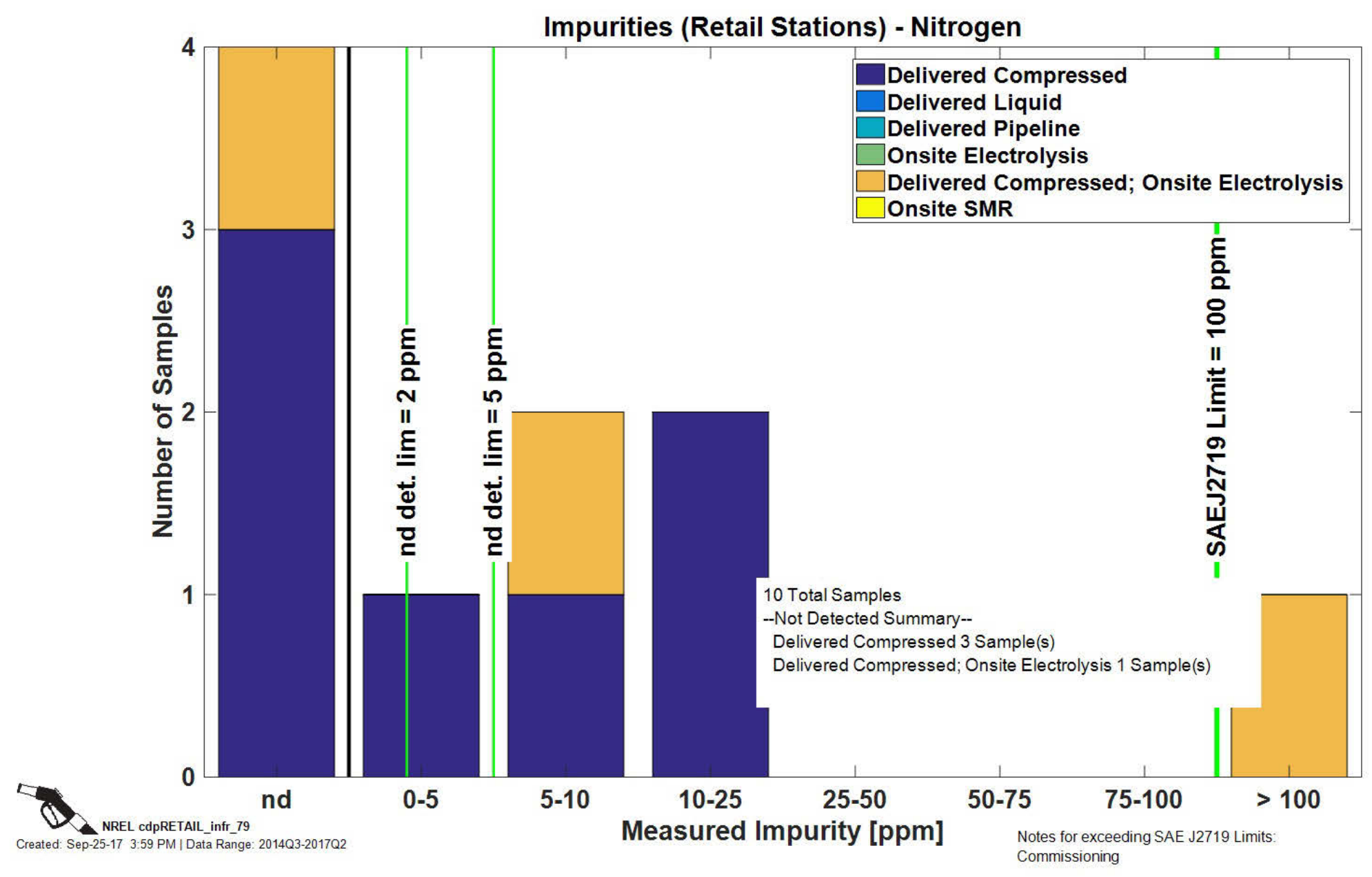




\section{CDP-INFR-79 \\ Impurities-Oxygen}

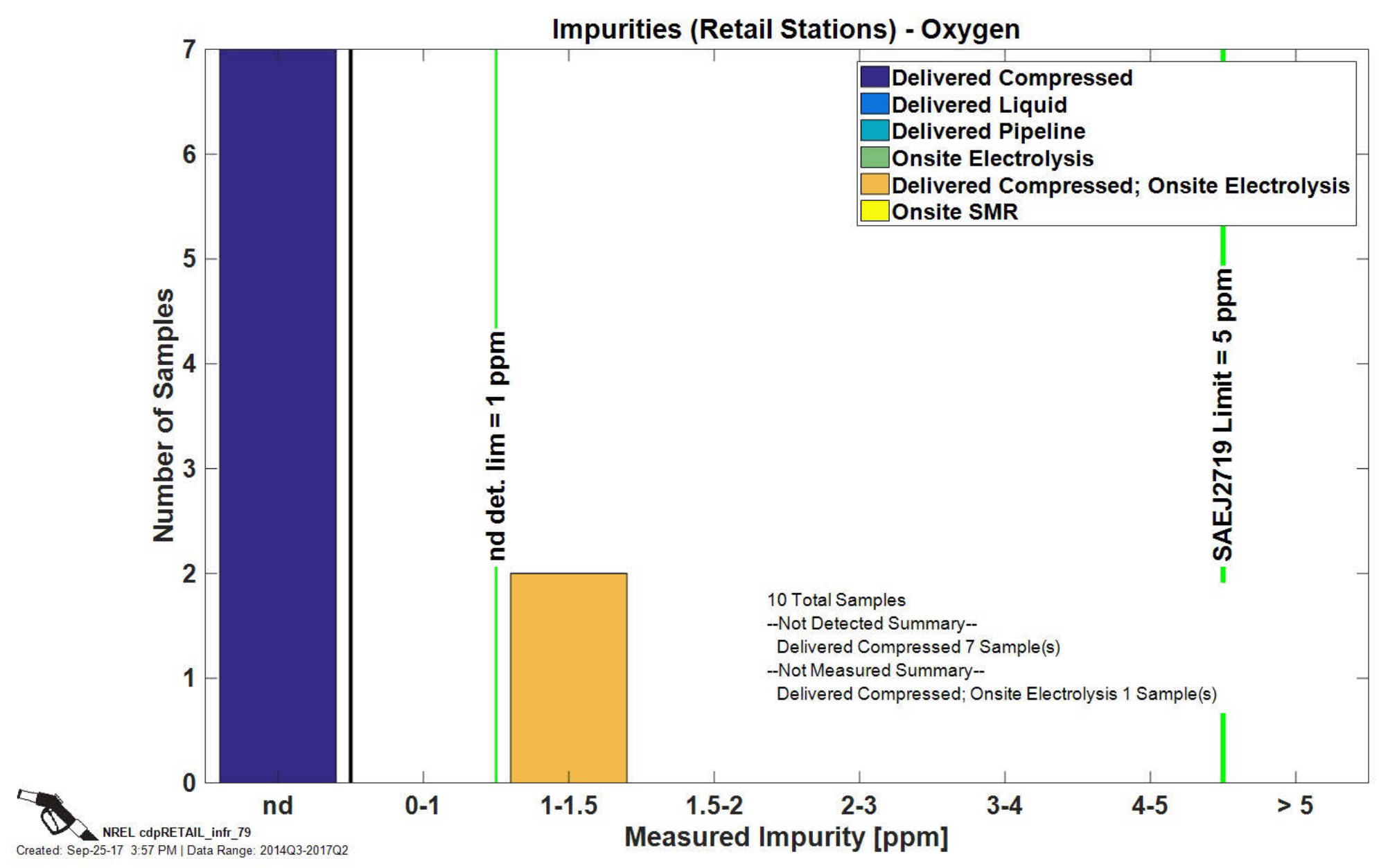




\section{Impurities-Particulate Concentration}

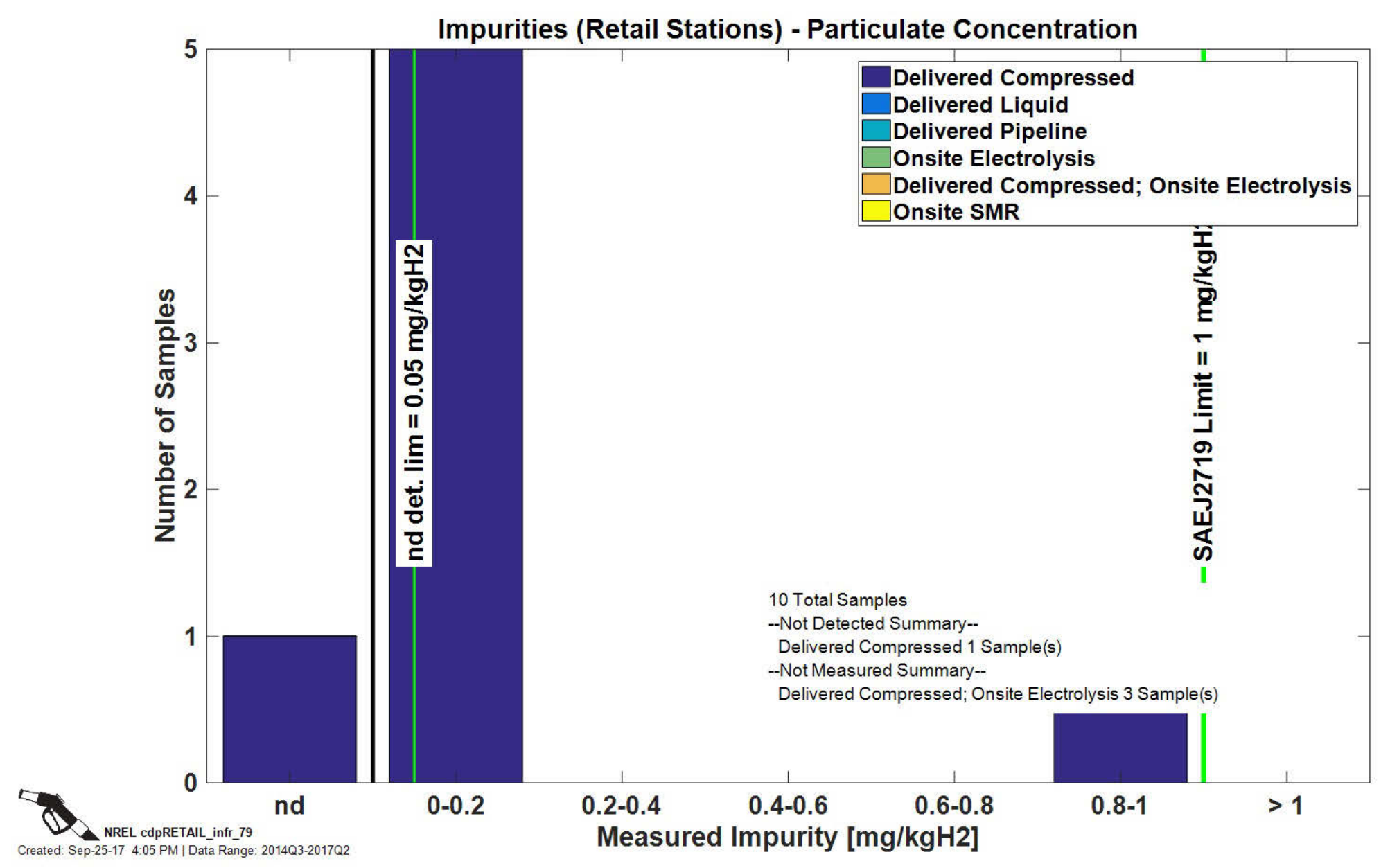




\section{Impurities - Total Halogenates}

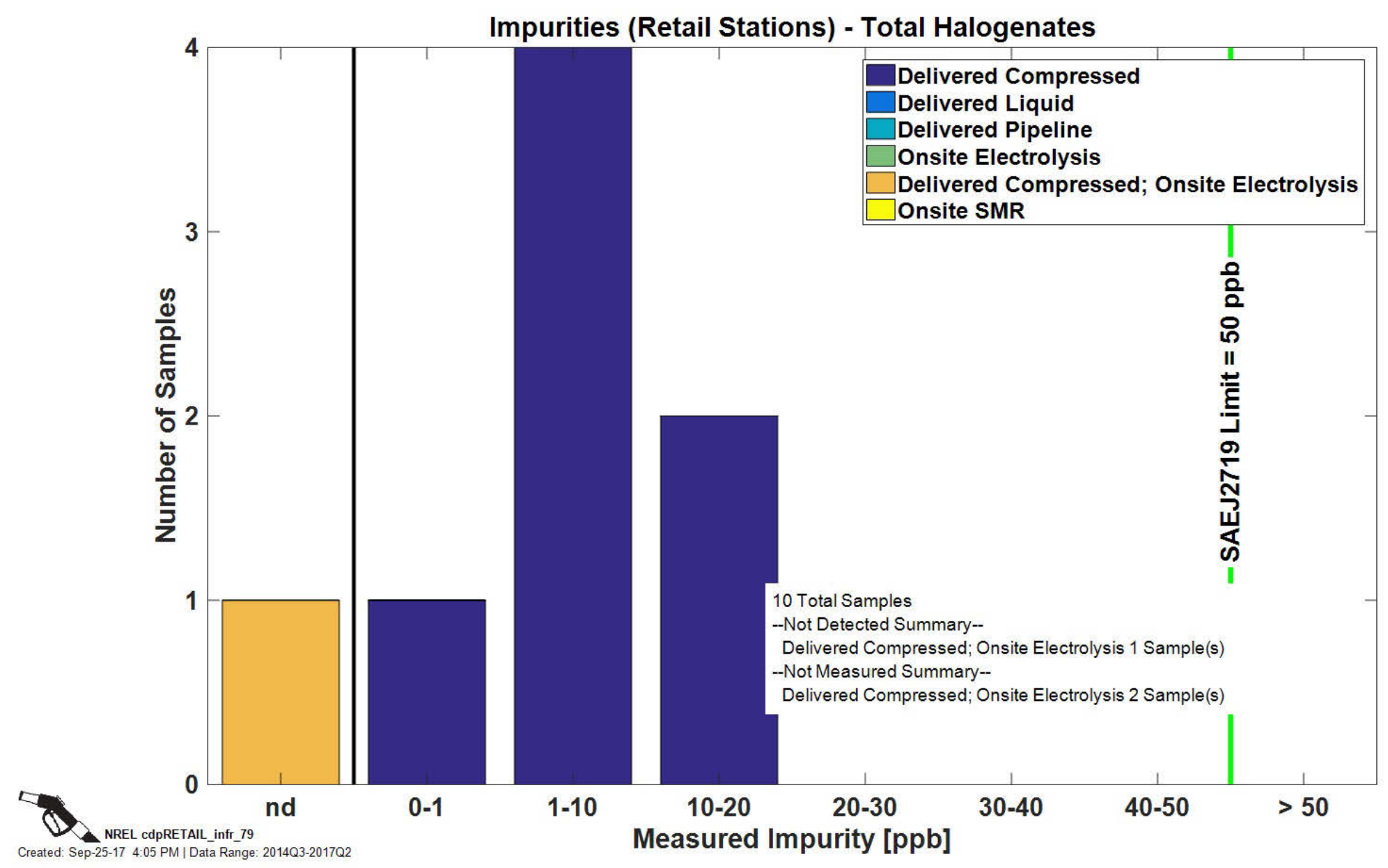




\section{Impurities-Total Hydrocarbons}

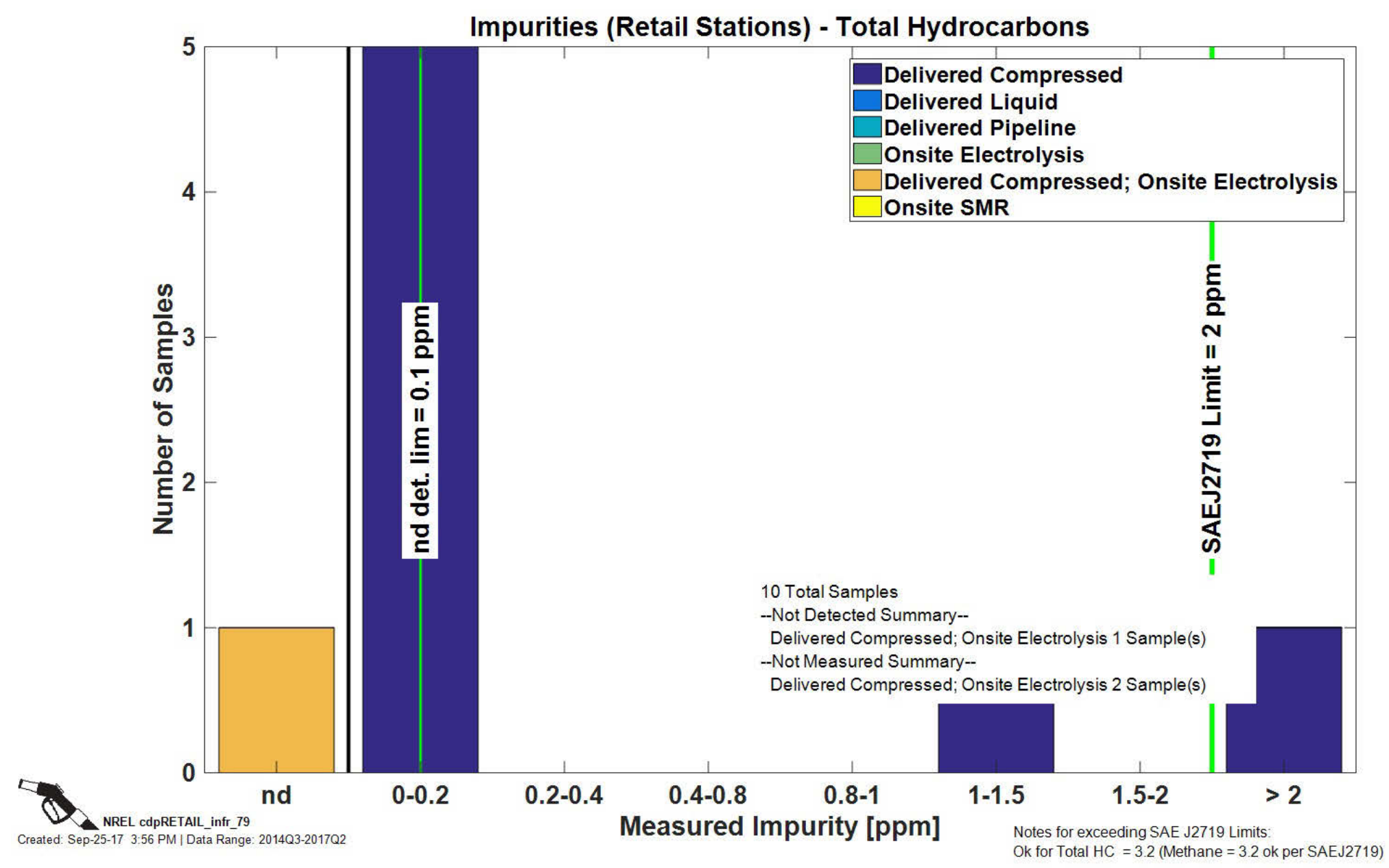




\section{CDP-INFR-79}

\section{Impurities-Total Sulfur}

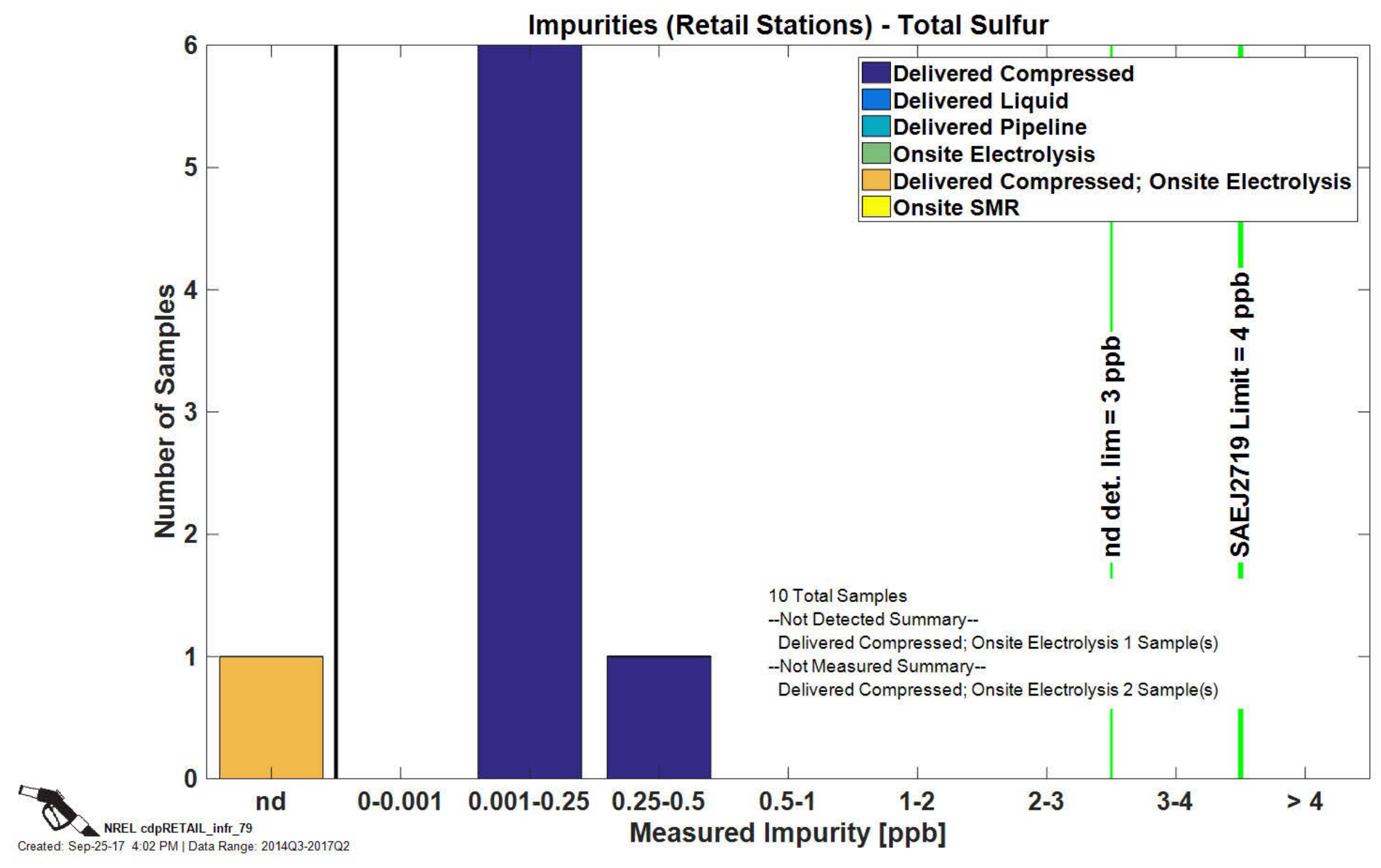




\section{CDP-INFR-79 \\ Impurities - Water}

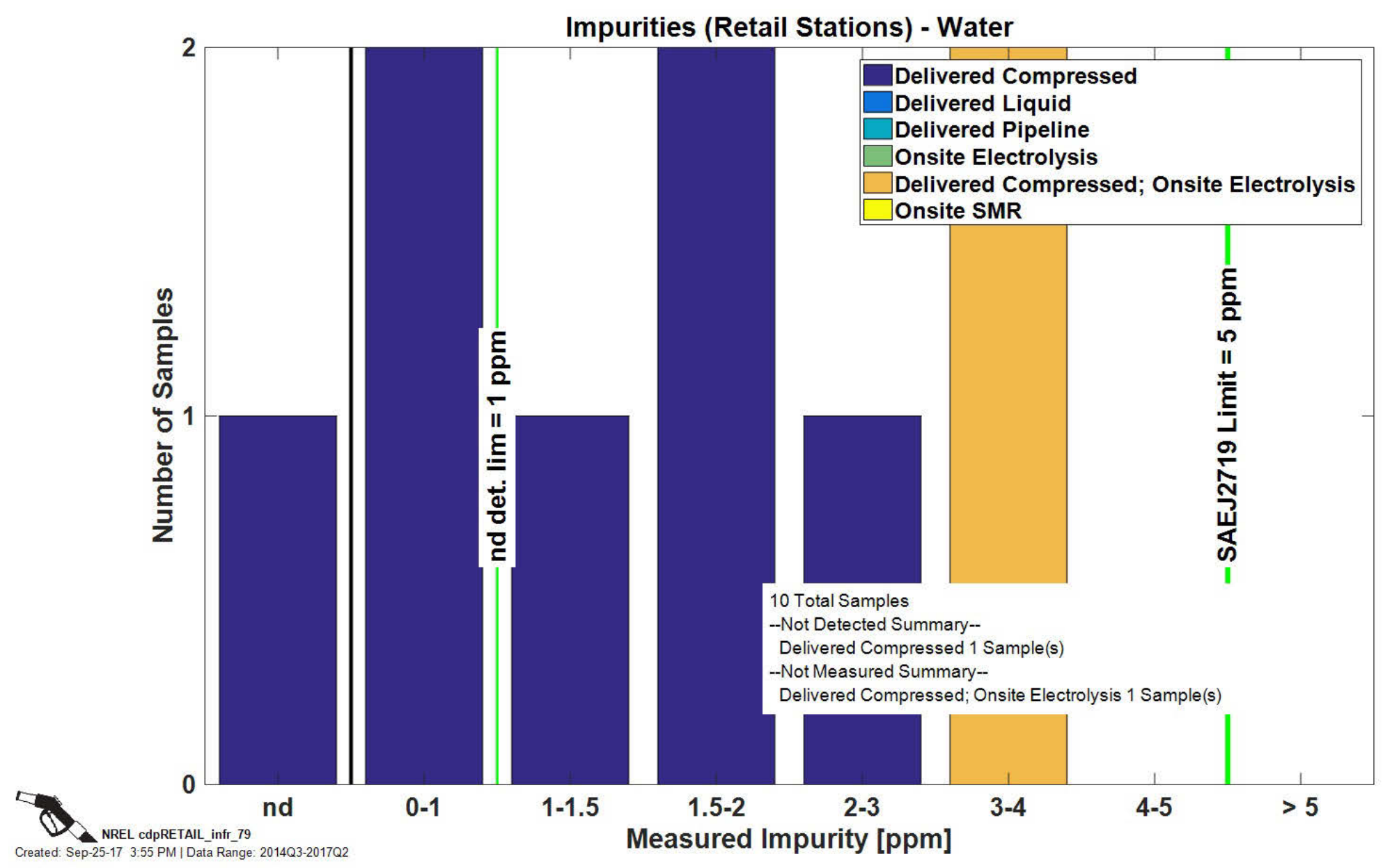


Component Energy 


\section{CDP-INFR-35 Compressor Energy}
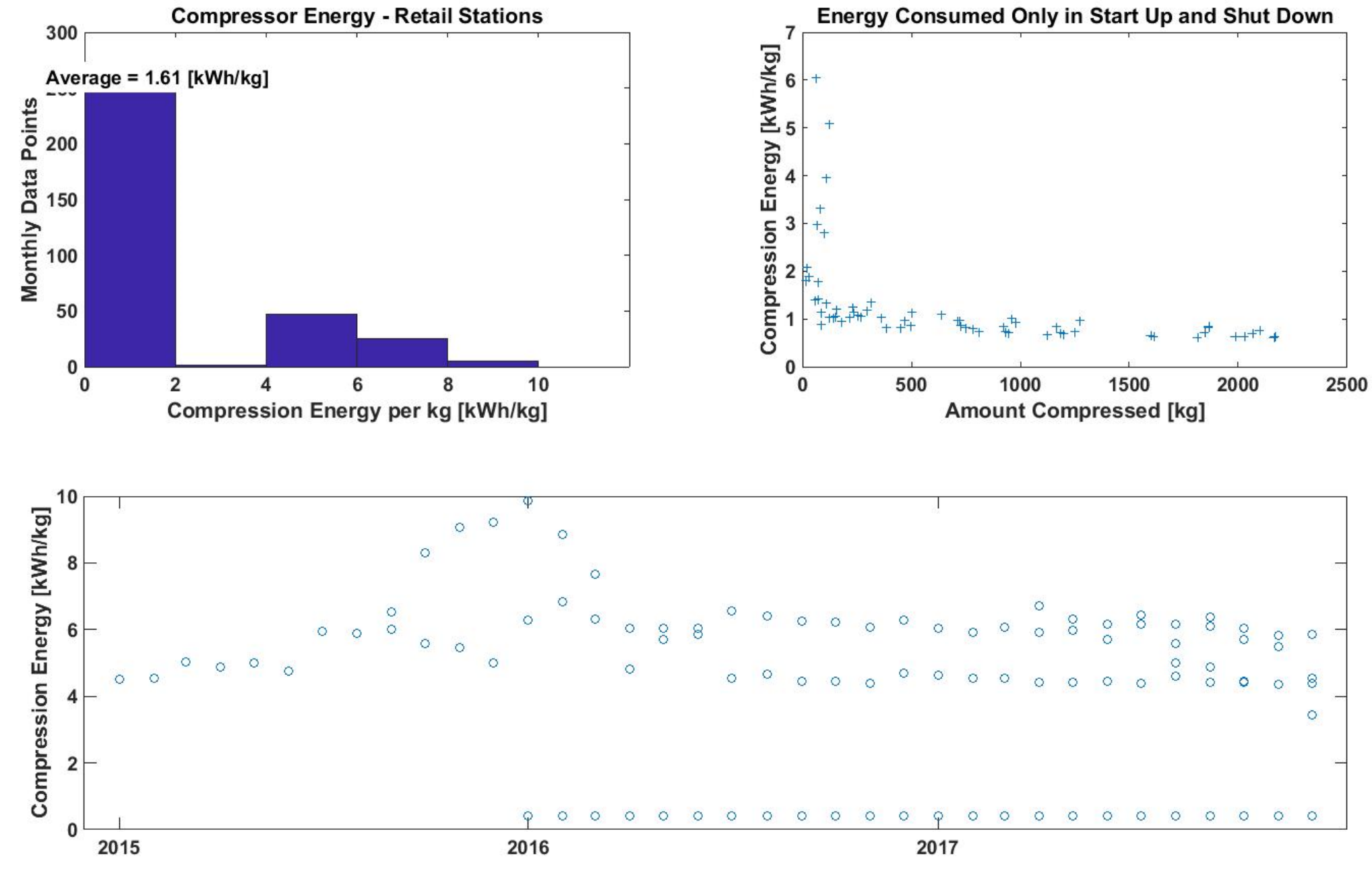

(1) NREL cdpRETAIL_infr_35

Created: May-07-18 10:33 AM | Data Range: 2014Q3-2017Q4 


\section{CDP-INFR-92 Dispenser Energy}
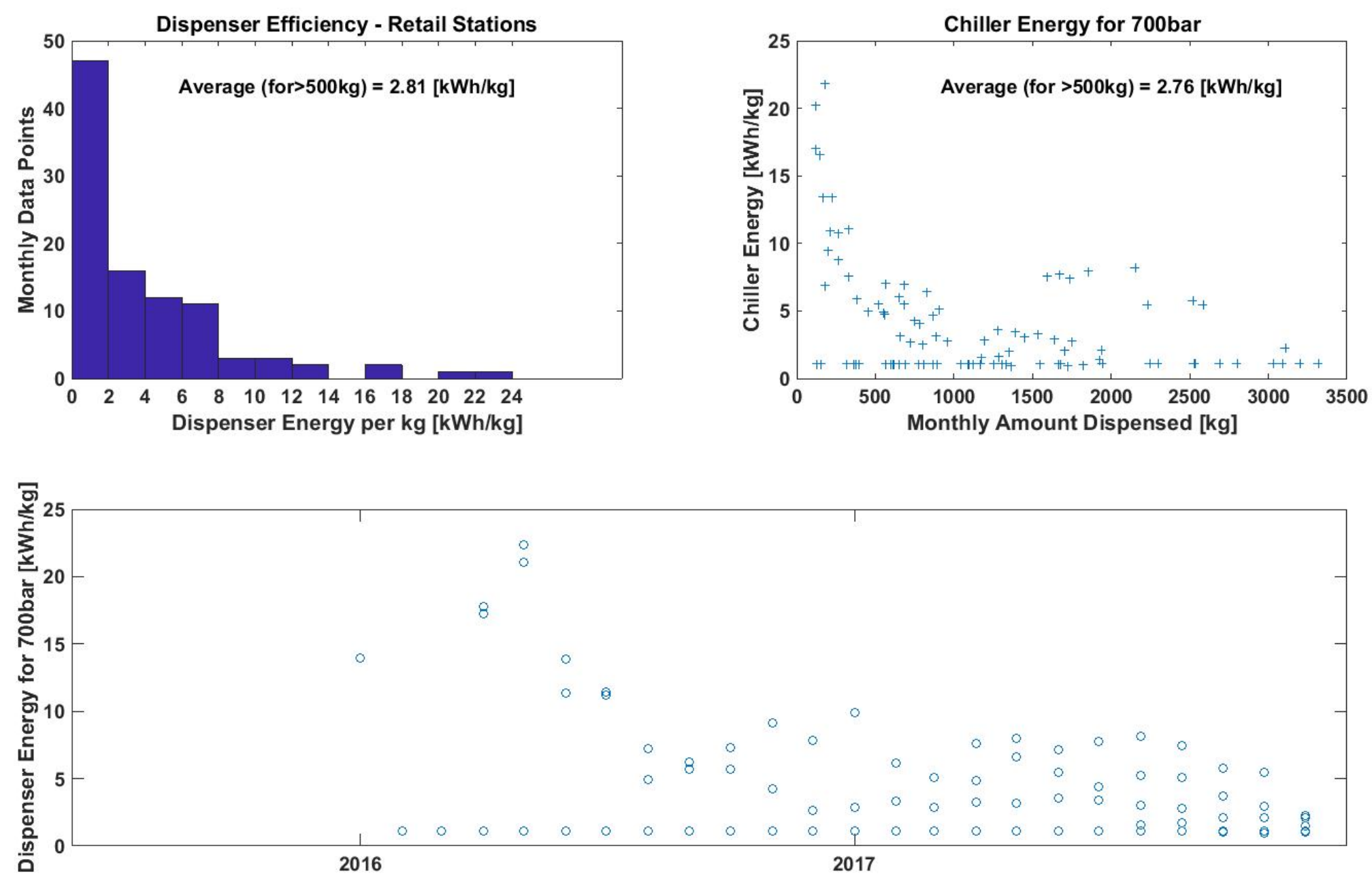


\section{CDP-INFR-78 \\ Station Energy per kg Dispensed}

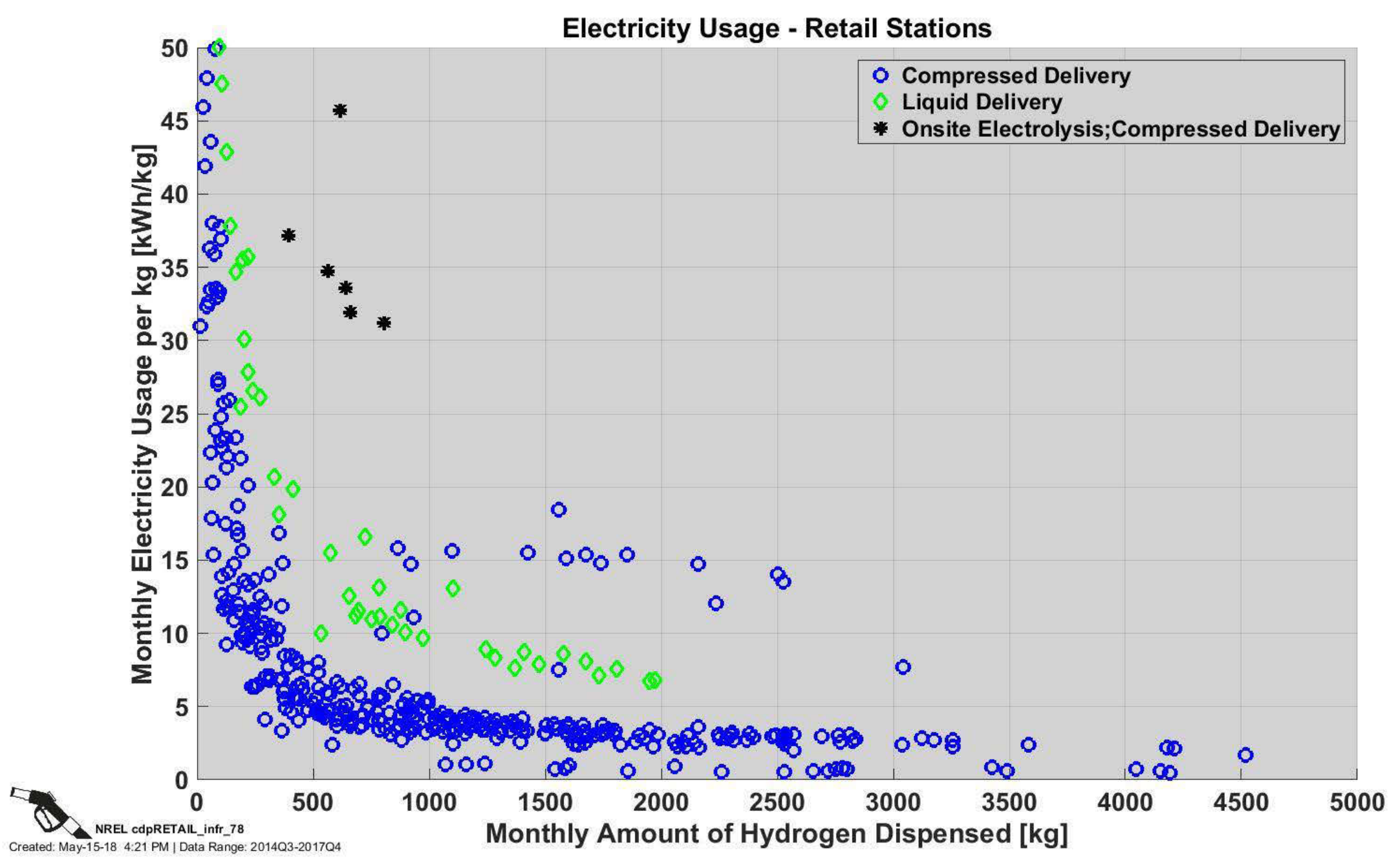




\section{CDP-INFR-83}

\section{Station Energy Cost per kg Dispensed}

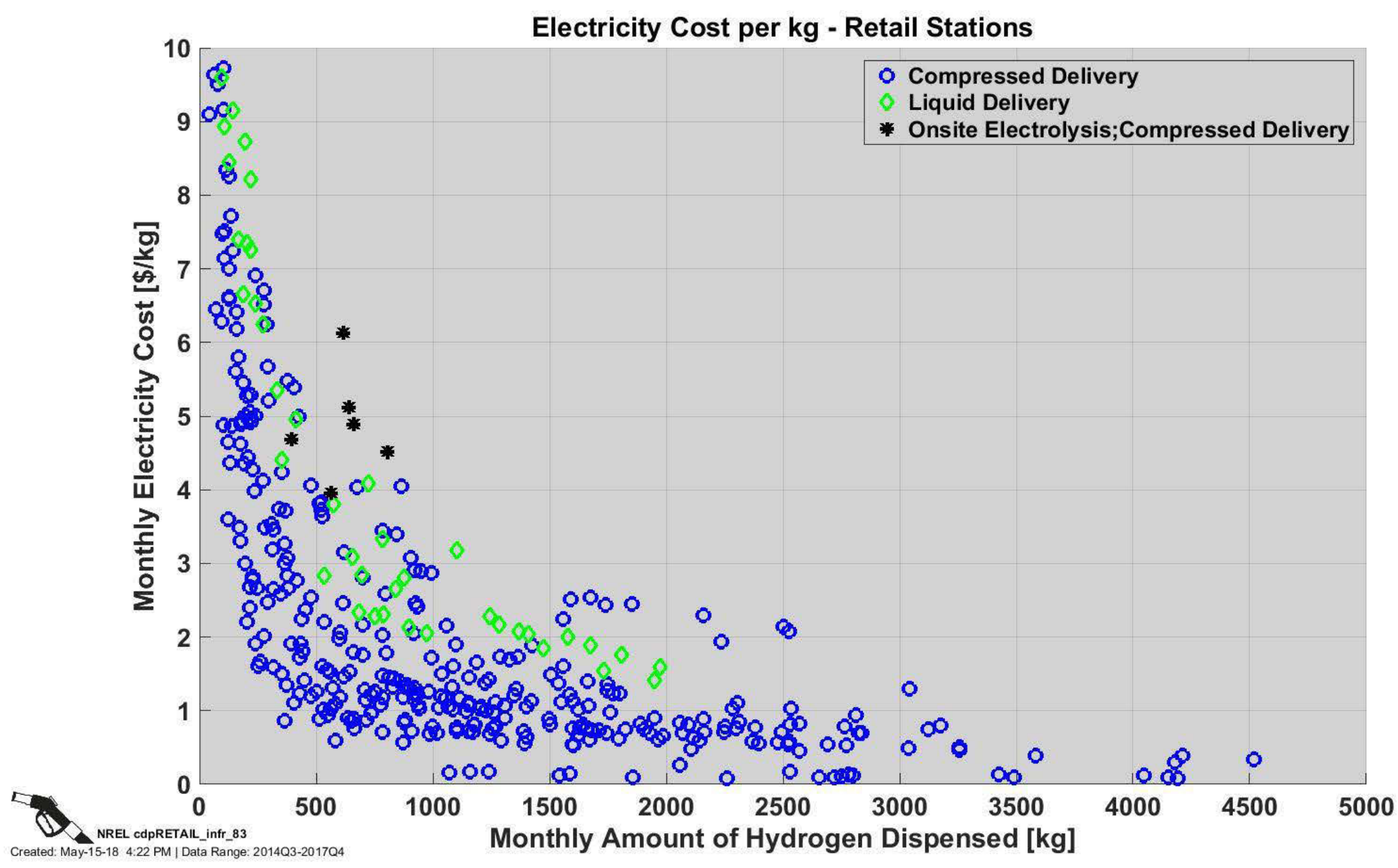




\section{CDP-INFR-84 \\ Station Electricity Cost per kWh}

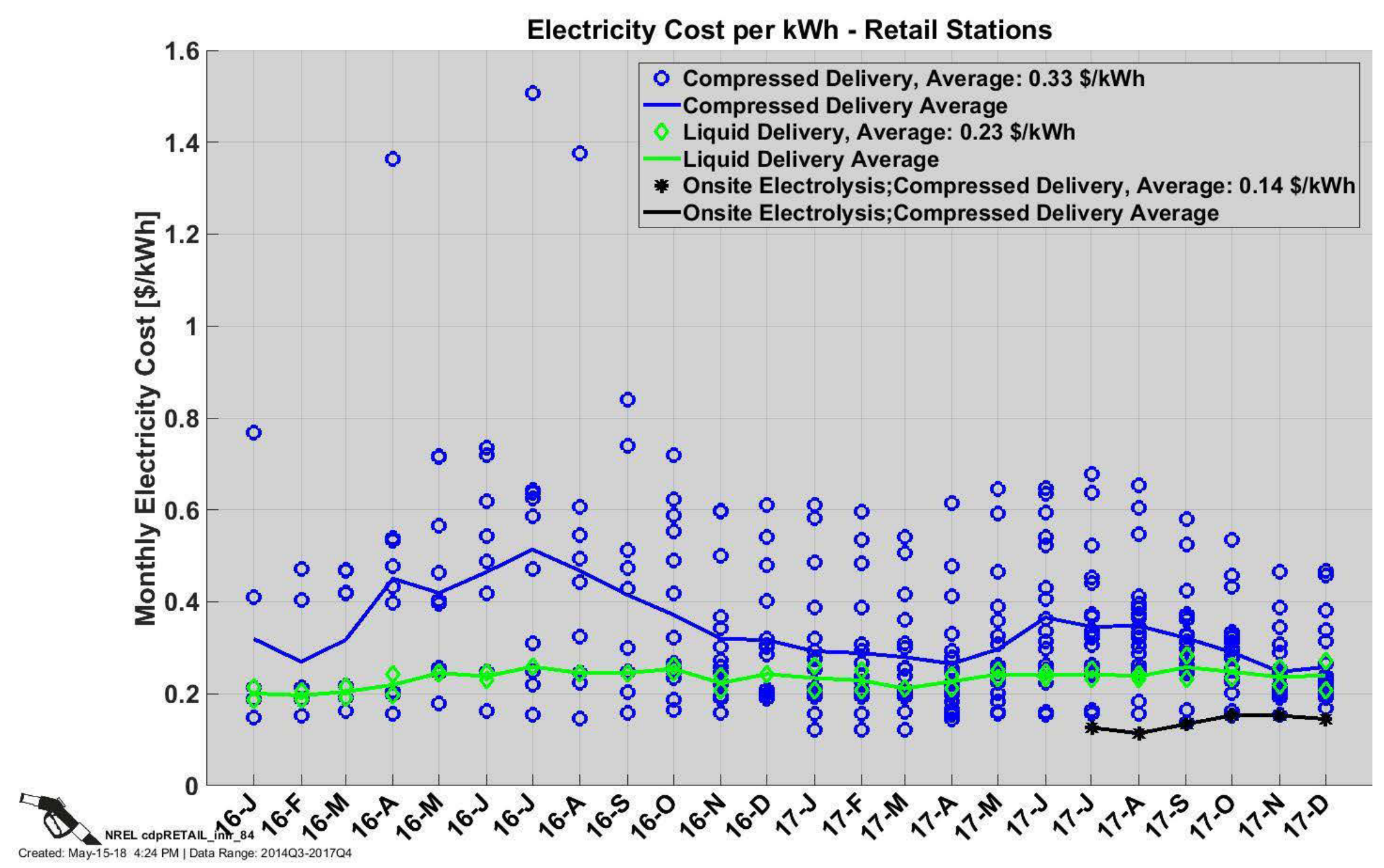




\section{CDP-INFR-85}

\section{Station Electricity Cost per kg Over Time}

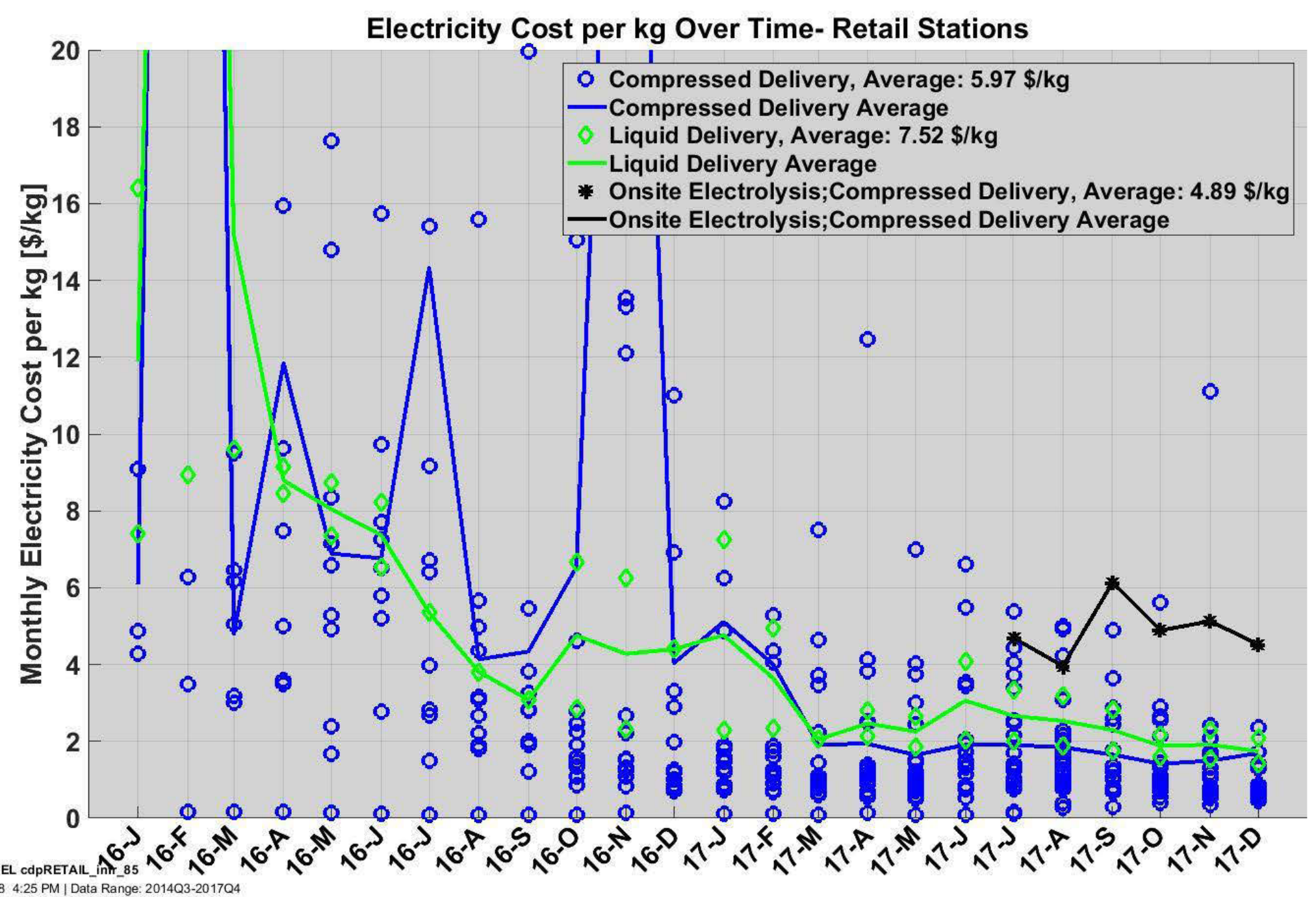




\section{CDP-INFR-86}

\section{Station Electricity Cost per kWh by Region}

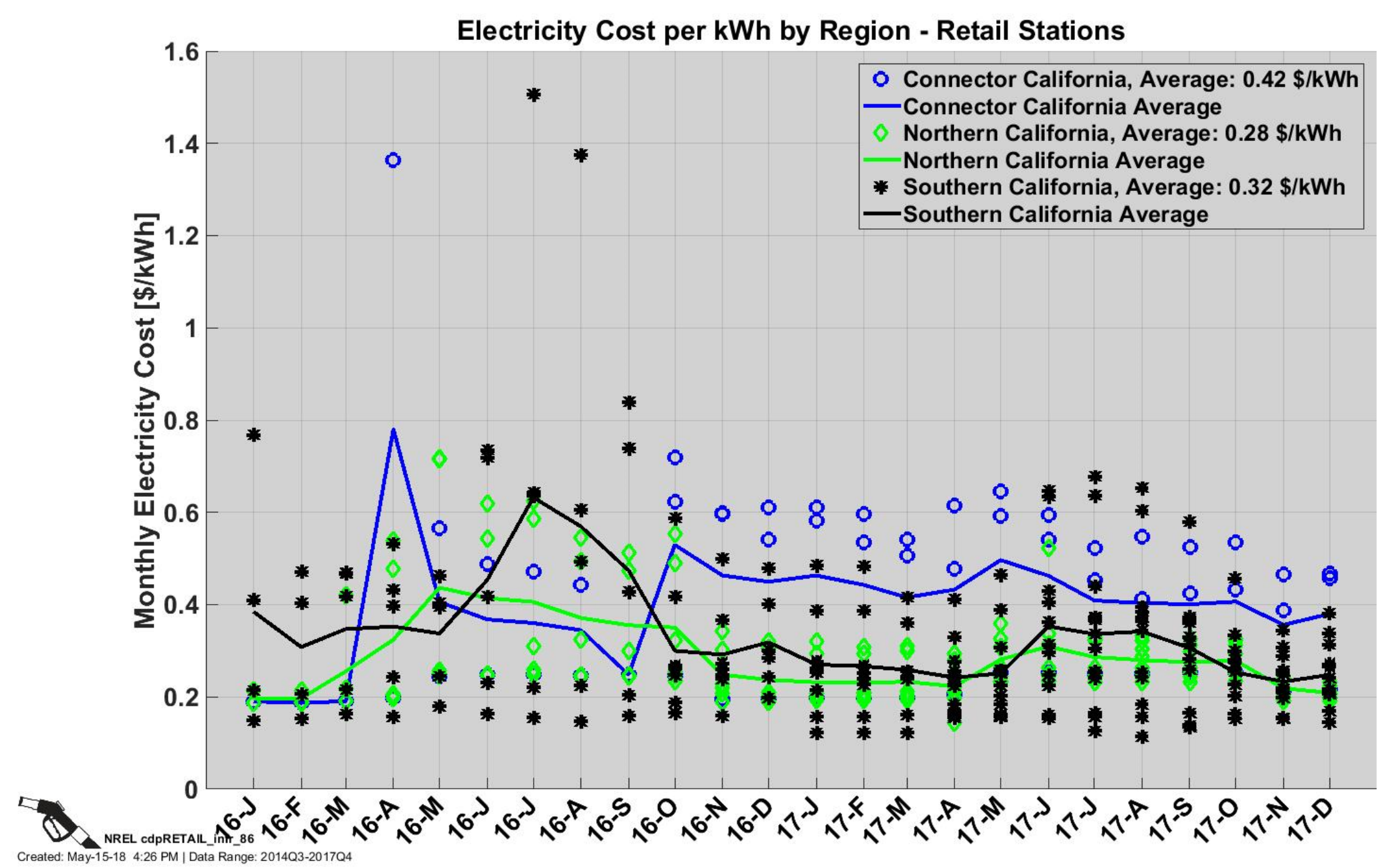




\section{CDP-INFR-87}

\section{Station Electricity Cost per kWh by Utility}

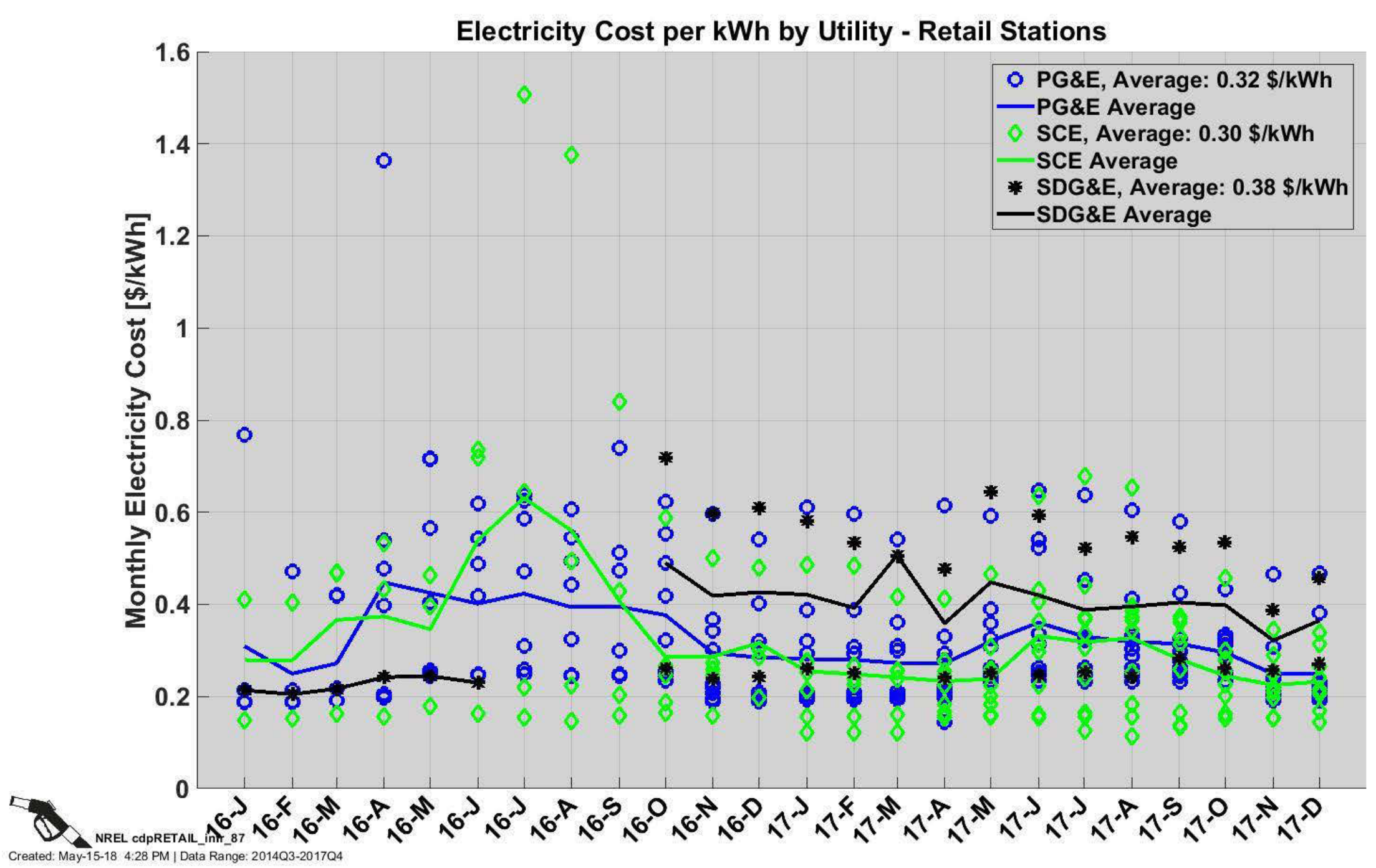




\title{
www.nrel.gov
}

\author{
NREL/PR-5400-71645
}

This work was authored by Alliance for Sustainable Energy, LLC, the manager and operator of the National Renewable Energy Laboratory for the U.S. Department of Energy (DOE) under Contract No. DE-AC36-08G028308. Funding provided by U.S. Department of Energy Office of Energy Efficiency and Renewable Energy Fuel Cell

Technologies Office. The views expressed in the article do not necessarily represent the views of the DOE or the U.S. Government. The U.S. Government retains and the publisher, by accepting the article for publication, acknowledges that the U.S. Government retains a nonexclusive, paid-up, irrevocable, worldwide license to publish or reproduce the published form of this work, or allow others to do so, for U.S. Government purposes.

\section{BNREL \\ NATIONAL RENEWABLE ENERGY LABORATORY}

